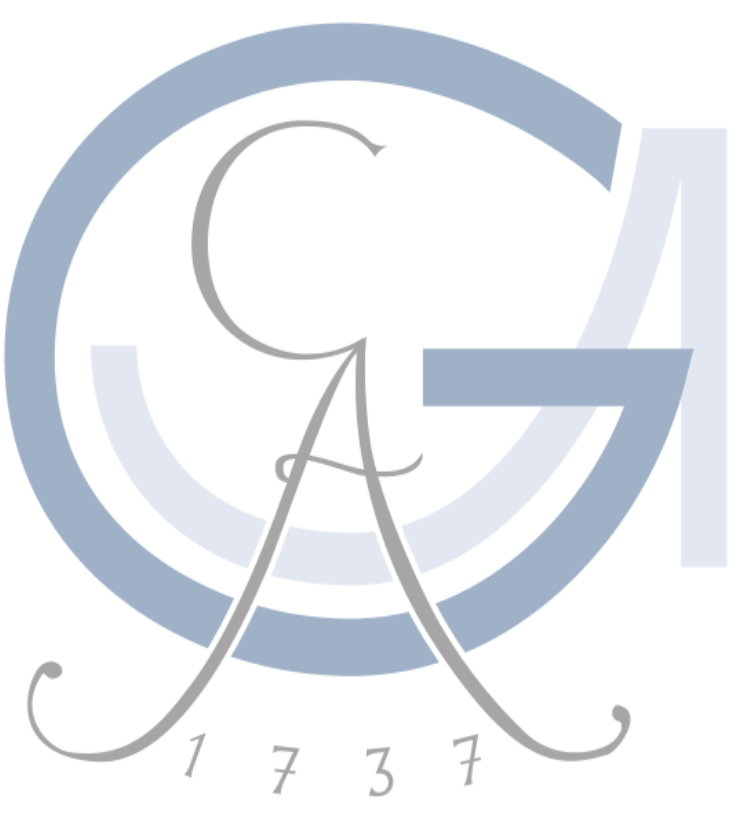

\title{
Self-organization of microtubules for biomimetic structures
}

\author{
Smrithika Subramani \\ born in Bangalore, India
}

Göttingen, 2019 



\title{
Self-organization of microtubules for biomimetic structures
}

\author{
Dissertation \\ to acquire the doctoral degree in mathematics and natural \\ science "Doctor rerum naturalium" at the \\ Georg-August-Universität Göttingen
}

within the doctoral degree program

Physics of Biological and Complex Systems

of the Göttingen Graduate School of Neurosciences,

Biophysics, and Molecular Biosciences (GGNB)

of the Georg-August University School of Sciences (GAUSS)

submitted by

Smrithika Subramani

from Bangalore, India

Göttingen 2019 


\section{THESIS COMMITTEE}

\section{Dr. Isabella Guido (Referee)}

Laboratory for Fluid Physics, Pattern Formation and Biocomplexity

Max Planck Institute for Dynamics and Self-Organization

Prof. Dr. Jörg Enderlein (Co-referee)

III. Physical Institute Biophysics/Complex Systems

Georg August University Göttingen

Prof. Dr. Stefan Klumpp

Institute for Nonlinear Dynamics

Georg August University Göttingen

\section{FURTHER MEMBERS OF THE EXAMINATION BOARD}

\section{Dr. Michael Wilczek}

Max Planck Research Group - Turbulence, Complex Flows and Active Matter Max Planck Institute Dynamics and Self-Organization

\section{Prof. Dr. Jörg Großhans}

Institute of Biochemistry and Molecular Cell Biology

Department of Developmental Biochemistry

University Medical Center Göttingen

\section{Dr. David Zwicker}

Max Planck Research Group - Theory of Biological Fluids

Max Planck Institute Dynamics and Self-Organization

Date of Oral examination: 14.10.2019 
One can't be satisfied with just looking at so wonderful a structure : chiefly because one can't get clear on how such an unbelievable motion is brought about.

Antony Van Leeuwenhoek, 1713 


\section{Contents}

1 Introduction 13

1.1 Microtubules : Assembly and dynamics . . . . . . . . . . . . . . . 15

1.2 Motor proteins : The molecular nanomachines . . . . . . . . . . . . 16

1.2.1 Kinesin-1 : Structure and stepping mechanism . . . . . . . . . 17

1.3 Synthetic active matter systems . . . . . . . . . . . . . . . . . . . . . 19

2 Materials and Methods 23

2.1 Polymerization of microtubules . . . . . . . . . . . . . . . . . . 23

2.1 .1 Confocal Laser Scanning Microscopy . . . . . . . . . . . . . . 24

2.2 Depletion agents induce MT bundling . . . . . . . . . . . . . . 25

2.3 Creating active microtubule bundles . . . . . . . . . . . . . . . 27

$2.3 .1 \quad$ Individual MT-motor protein bundles . . . . . . . . . . . . . 29

2.4 A modified gliding assay . . . . . . . . . . . . . . . . . . . . . . . . . 29

2.5 Networks under geometrical confinement . . . . . . . . . . . . . . . 30

2.6 Surface passivation techniques . . . . . . . . . . . . . . . . . 31

2.6 .1 Coating PLL-PEG on glass coverslips . . . . . . . . . . . . . . 32

2.6 .2 Coating silane-PEG on glass coverslips . . . . . . . . . . . . 33

2.7 Surface attachment via nucleation templates . . . . . . . . . . . . . . 33

2.7 .1 Microseeds as nucleation initiators . . . . . . . . . . . . . 34

2.7 .2 MAP as an anchor system . . . . . . . . . . . . . . 34

2.7 .3 The UV Micropatterning technique . . . . . . . . . . . . . . . 35

2.8 Computational methods . . . . . . . . . . . . . . . . . . . . . . 38

$2.8 .1 \quad$ Filament tracking using Jfilament . . . . . . . . . . . . . . . . 38

2.8 .2 Particle tracking algorithms . . . . . . . . . . . . . . . . . . 38

2.8 .3 Orientation measurements using OrientationJ . . . . . . . . . 40

2.8 .4 Estimation of flexural rigidity . . . . . . . . . . . . . . . 40

3 Results 45

3.1 Kinesin-embedded microtubule networks . . . . . . . . . . . . . . . . 46

3.2 Bending dynamics of individual bundles . . . . . . . . . . . . . . 52

$3.2 .1 \quad$ Experimental results . . . . . . . . . . . . . . . . 52 
3.3 Effects of geometrical confinement of

MT-motor mixtures . . . . . . . . . . . . . . . . . . . . . . . 57

$3.3 .1 \quad 10 \mu \mathrm{m}$ tube-polymerized . . . . . . . . . . . . . . 58

$3.3 .210 \mu \mathrm{m}$ chamber-polymerized . . . . . . . . . . . . . . . 62

$3.3 .3 \quad 100 \mu \mathrm{m}$ tube-polymerized . . . . . . . . . . . . . . 64

$3.3 .4100 \mu \mathrm{m}$ chamber-polymerized . . . . . . . . . . . 66

3.4 Oscillatory behavior of a single microtubule . . . . . . . . . . . . 68

3.4 .1 Experimental results . . . . . . . . . . . . . . . . . . . . 69

3.4 .2 Theoretical discussion $\ldots \ldots \ldots$. . . . . . . . . . 73

3.5 Surface attachment of MTs . . . . . . . . . . . . . . . . . 74

$\begin{array}{lll}4 & \text { Discussion } & 77\end{array}$

4.1 Motor cluster behaviour in networks . . . . . . . . . . . . . . . 77

4.2 Bundled active networks . . . . . . . . . . . . . . . . . . . . . . . 79

4.3 Single active filament behaviour . . . . . . . . . . . . . . . . . 81

4.4 Towards synthetic reconstitution of cilia . . . . . . . . . . . . . 82

4.5 Conclusion and Outlook . . . . . . . . . . . . . . . . . . . . . . 83 


\title{
Self-organization of microtubules for biomimetic structures
}

\begin{abstract}
Biological polymers that build up the eukaryotic cytoskeleton exhibit a rich dynamical behaviour that aid a multitude of cellular processes. Microtubules, together with their associated motor proteins in the cell are involved in cell motility, mitosis, intracellular transport and maintaining cellular rigidity. In the recent past, synthetic microtubule-motor protein systems have been extensively studied owing to their collective display of higher-level self-organization through spontaneous beating, dynamic pattern formation in dense suspensions and enhanced transport through cytoplasmic streaming.

In this work, we focus on understanding microtubule-motor protein interactions through an in vitro bottom-up approach. We gain information on the motor proteininduced dynamics on pairs or bundles of sliding microtubule bundles as well as crosslinked microtubule networks. The activity due to bending, buckling, merging and disassembly of active bundles are studied concerning their dilute as well as crowded environments. The effects of kinesin-induced activity in translating active bundled networks from a coherent to a turbulent phase of active flows is studied through geometrical confinement. The influence of confinement heights on the selfassembly processes of microtubule bundles and their effective length distributions are studied. The study of such assays as active matter systems aids us in understanding motor transport at various levels of microtubule organization. Moving to a dilution limit to obtain just a single pinned microtubule filament, we were able to observe oscillatory behaviour due to a continuous attachment-detachment cycle between motor proteins and the microtubule. This simple beating serves as a minimal system to understand the complex mechanism behind fluid manipulation phenomena such as ciliary beating and intracellular streaming. We define microtubules and motor proteins as self-assembling, versatile building blocks to explain their roles in biological structures.
\end{abstract}




\section{List of Figures}

1.1 Internal structure of cilia . . . . . . . . . . . . . . . . . . . . . . 14

1.2 Microtubule assembly . . . . . . . . . . . . . . . . . . . . . . . 15

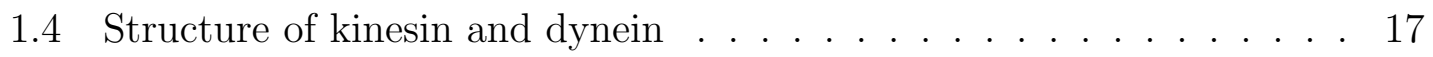

1.5 Kinesin stepping . . . . . . . . . . . . . . . . . . 18

$1.6 \quad$ Schematic depiction of gliding and stepping assays . . . . . . . . . . . 20

1.7 Building blocks for cilia . . . . . . . . . . . . . . . . . . . . . . 21

$2.1 \quad$ Polymerized microtubules $\ldots \ldots \ldots \ldots$. . . . . . . . . . . . . . . 24

2.2 Mechanism of the Depletion effect . . . . . . . . . . . . . 26

2.3 Geometrically confined MT-motor protein networks . . . . . . . . . . 30

2.4 PLL-PEG functionalization . . . . . . . . . . . . . . . . . . . . . 32

2.5 MT attahment via MAP . . . . . . . . . . . . . . . . . . . . . 35

2.6 Micropatterns $\ldots \ldots \ldots \ldots$

2.7 Micropatterning procedure 1 . . . . . . . . . . . . . . . . . 37

2.8 Micropatterning procedure $2 \ldots \ldots \ldots$

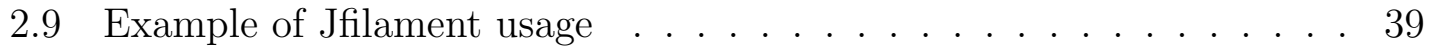

2.10 Worm-like chain model . . . . . . . . . . . . . . . . . . . . . . . . . . 40

2.11 Thermally fluctuating microtubules . . . . . . . . . . . . . . . . . 43

3.1 Tracking of kinesin clusters . . . . . . . . . . . . . . . . . . . . 47

$3.2 \quad$ Histograms of total travel length and average active velocities of kinesin clusters . . . . . . . . . . . . . . . . . . . . . . . . . . . . . . . . . 48

3.3 Illustration of activity vs association . . . . . . . . . . . . . . 48

3.4 Histogram of activity time and activity length . . . . . . . . . . . . . 49

3.5 Association time and association length . . . . . . . . . . . . . . 50

$3.6 \quad$ Dynamics of freely moving MT-motor bundles . . . . . . . . . . . . . 53

3.7 Active bending length of freely moving MT-motor bundles . . . . . . 54

3.8 Force density calculations for freely moving MT-motor bundles . . . . 55

3.9 Histogram of force densities of freely moving MT-motor bundles . . . 55

3.10 Multi-plane imaging of MT-motor bundles . . . . . . . . . . . . 56 
3.11 The chaotic, transient and directed phase of MT-motor dynamics in a $10 \mu \mathrm{m}$ high chamber . . . . . . . . . . . . . . . . . . 59

3.12 Nematic order parameter $(S)$ as a function of time . . . . . . . . . 60

3.13 Time-averaged MSD behaviour of the three phases in $10 \mu \mathrm{m}$ tubepolymerized MT bundle-motors . . . . . . . . . . . . . . . . . . . . . 61

3.14 Length distributions of tube-polymerized MT bundles in a $10 \mu \mathrm{m}$ chamber . . . . . . . . . . . . . . . . . 6 62

3.15 Particle trajectories of $10 \mu \mathrm{m}$ chamber-polymerized bundles . . . . . 62

3.16 MSD behaviour of $10 \mu \mathrm{m}$ chamber-polymerized MT bundles . . . . . 63

3.17 Length distribution of $10 \mu \mathrm{m}$ chamber-polymerized MT bundles . . . 64

3.18 Time evolution of tube-polymerized MT bundles in $100 \mu \mathrm{m}$ chambers 64

3.19 Length distribution of tube-polymerized MT bundles in a $100 \mu \mathrm{m}$

chamber . . . . . . . . . . . . . . . . . 65

3.20 Particle imaging velocimetry (PIV) velocity maps of $100 \mu \mathrm{m}$ tube-

polymerized bundles exhibit large-scale vorticities . . . . . . . . . . 65

3.21 Time-lapse and PIV velocity maps of $100 \mu \mathrm{m}$ chamber-polymerized

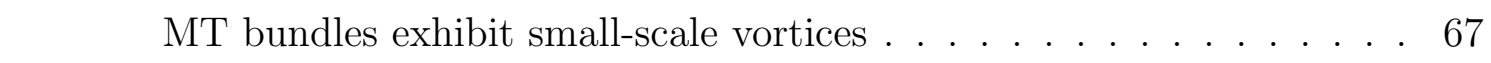

3.22 Length distribution of $100 \mu \mathrm{m}$ chamber-polymerized MT bundles . . 68

3.23 Comparison of average bundle lengths in the four configurations . . . 68

3.24 Schematic representation of clamped MT with motors . . . . . . . . 69

3.25 Oscillation cycles of a single clamped microtubule . . . . . . . . . . 70

3.26 (A) The time evolution of oscillations can be tracked as seen in various

\begin{tabular}{|c|}
\hline colors. (B) the tangent angle $\psi(s, t)$ as a function of the arc length $s$ \\
\hline
\end{tabular}

along the filament [104]. . . . . . . . . . . . . . . . . 71

3.27 Time-evolution of the tangent angle along the filament represents

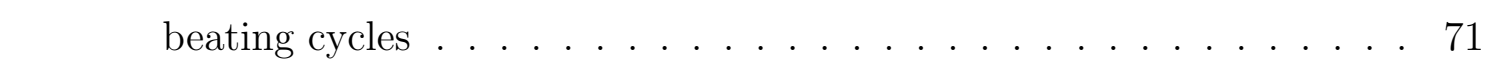

3.28 Control experiments to verify the optimum motor concentrations for

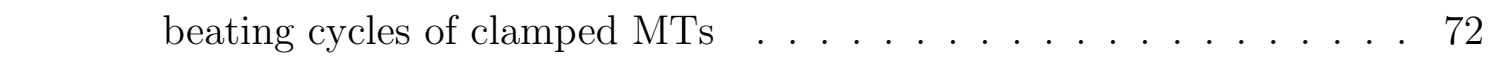

3.29 The tangent - tangent correlation of the persistently oscillating filament 73

3.30 The reconstruction of the oscillating filament's shape with the corre-

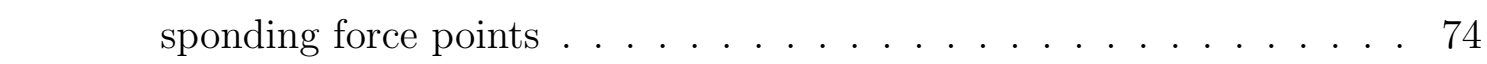

3.31 (A) MTs attached via MAP on micropillars. Arrows indicate the

\begin{tabular}{|c|}
\hline direction of applied flow. Scale bar: $10 \mu \mathrm{m}$ (B) Microtubules attached \\
\hline
\end{tabular}
via biotin-neutravidin on stripe, square and dot patterns. Scale bar : $10 \mu \mathrm{m} \ldots \ldots \ldots \ldots \ldots 75$

$4.1 \quad$ Schematic illustration of the three experimental scenarios described in this work . . . . . . . . . . . . . . . . . . . . . 82 


\section{List of Tables}

2.1 Components of active MT mixture . . . . . . . . . . . . . . . . 28

2.2 MT bundle polymerization mixture . . . . . . . . . . . . . . . . . . . 29

2.3 MT seeds preparation . . . . . . . . . . . . . . . . . . . 34 


\section{Glossary}

- MT : Microtubules

- WLC : Worm-like chain

- CSF : Cerebrospinal fluid

- PIPES : piperazine-N,N-bis(2-ethanesulfonic acid)

- $\mathbf{K O H}$ : Potassium hydroxide

- EGTA : (ethylene glycol-bis( $\beta$-aminoethyl ether)-N,N,N,N-tetraacetic acid)

- MgCl2 : Magnesium chloride

- BRB80/M2B : Brinkley buffer

- CLSM : Confocal Laser Scanning Microscopy

- PEG : poly(ethylene)glycol

- ATP : Adenosine Triphosphate

- GTP : Guanosine -5-triphosphate

- GMPCPP : Guanosine-5-[( $\alpha, \beta)$-methyleno]triphosphate

- PEP : Phosphoenol pyruvate

- PK/LDH : Pyruvate kinase lactic dehydrogenase

- GO : Glucose oxidase

- DTT : Dithiothreitol

- PLL-g-PEG : Poly(L-lysine)-graft-poly(ethylene glycol)

- mPEG-silane : methoxy -poly(ethylene glycol)silane

- HEPES : 4-(2-hydroxyethyl)-1-piperazineethanesulfonic acid 
- MAP : Microtubule Associated Protein

- PIV : Particle imaging Velocimetry

- MSD : Mean Squared Displacement 


\section{Chapter 1}

\section{Introduction}

The eukaryotic cell, amidst its diversity in form and function, forms the most fundamental unit in the various hierarchical levels of biological organization. However, the cell itself is one of the most complex, highly organized dynamic systems built from interactions of molecular components. Its rigidity and shape are maintained by the cytoskeleton. It accounts for the mechanical properties of cells and sub-cellular structures such as nerve cells, bending sperm tails and beating cilia on ependyma surfaces. Their working is controlled through the interactions between three individual cytoskeletal filament systems: microtubules (referred to as MTs hereafter), actin (microfilaments) and intermediate filaments.

MTs in the cell perform several crucial functions such as (i) cell division, where they form the mitotic spindle to separate replicated chromosomes, (ii) cell transport, by moving organelles around and lending cellular shape and structure and (iii) cell movement, where they form a part of the framework of extracellular appendages such as cilia and flagella. This work shall focus on the transport action of MTs as a part of ciliary arrays that beat synchronously to propel fluids at sub-millimetre scales through the action of motor proteins. During important processes such as mitosis, the MTs within a spindle are engaged in large amounts of protein trafficking.

At the extracellular level, the beating of cilia occurs in a symmetric fashion through an effective stroke (straight cilia) and subsequent recovery stroke (curved cilia) that is known to maximize transport. They are present on the ependyma of mammalian brains to transport cerebrospinal fluid (CSF), on the lining of the trachea and human lungs to sweep out mucus and dirt out of the airways. Even the fallopian tubes of females are covered with cilia that move the fertilised ovum from the ovary to the uterus, where the ovum attaches itself [49].

The internal structure of the cilium is quite complex, containing at least 600 different types of proteins [77, 54]. It consists of a flexible cylindrical structure called the axoneme that holds nine pairs of MT doublets at the periphery and one 
pair of microtubules at the centre, making it a $(9+2)$ axoneme. The nine outer MT

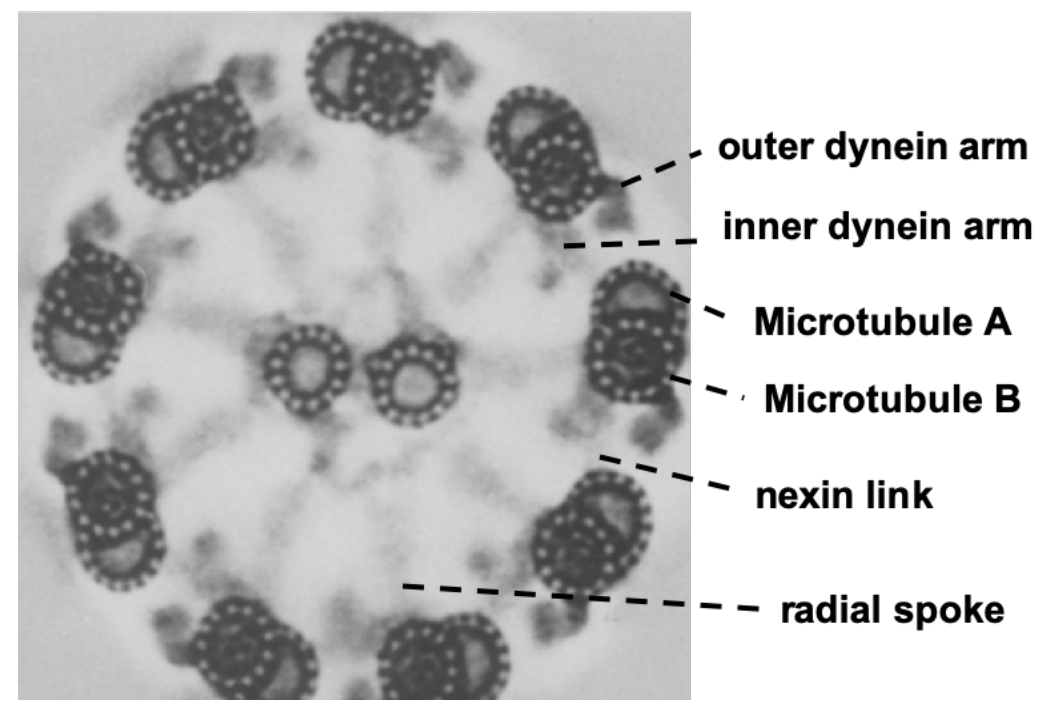

Figure 1.1: Electron micrograph of the cross section of a $(9+2)$ axoneme, taken from [1].

doublets are connected through nexin links while they are connected to the central pair through radial spokes. The outer MTs also have inner and outer arms of motor proteins called dyneins (Figure 1.1). During the absence of ATP, the dyneins at the outer MT pairs are attached adjacently to each other. During ATP hydrolysis, the dyneins get detached, move and re-attach in a way that pairs of neighbouring MTs begin to slide. Since these MTs are connected through nexins and radial spokes, their gliding motion contributes to an overall elastic bending of the cilium. This motor protein action initiates travelling waves that propel the cilium by overcoming the viscous forces of the surrounding fluid.

At the intracellular level, MT-motor protein interactions can cause cytoplasmic streaming, a method of fluid transport reported in larger cells such as Drosophila oocytes [101, 29] and Elodea leaf cells [5]. This emergence of a regimented flow hints at an autonomous process of MT-motor self-organization that dynamically drives the formation of streaming patterns [36]. Through this process, fluid flow and mixing occurs to speed up molecular and organelle transport in the cell. It also aids the uniform distribution of nutrients and development of the zygote [87, 61]. Although the streaming phenomenon is driven by motor-induced forces, the underlying relationship between motor activity and the observed flows is not understood clearly.

In this work, we work on a bottom-up approach towards understanding transportdriven processes such as ciliary beating and cytoplasmic streaming through the synthetic reconstitution of MTs and motor proteins as building blocks. We study minimal systems containing these building blocks that can generate cilia-like oscil- 
latory behavior and self-assemble as active matter systems. The minimal in vitro reconstitution approach that we adopt can offer a deeper understanding of biological phenomena that are complex to investigate in vivo. In this regards, the remainder of this chapter shall focus on introducing concepts related to MT structure and dynamics, the working of motor proteins (with a specific focus on kinesin) and the recent evolution of MT-motor protein assemblies into synthetic active matter systems.

\subsection{Microtubules : Assembly and dynamics}

MTs are hollow polymer-cylinders of outer diameter $25 \mathrm{~nm}$ and tens of $\mu \mathrm{m}$ long characterized by high stiffness (persistence length in $\mathrm{mm}$ ) that play an important role in cell division and vesicle transport [3]. MTs are made up of molecular units called tubulin; these heterodimer subunits contain $\alpha$ - and $\beta$-tubulin monomers (each of molecular mass $\sim 50 \mathrm{kDa}$ and repeat length $8 \mathrm{~nm}$ ) [12] that interact non-covalently in a head-to-tail fashion with more tubulin subunits to form a protofilament.

This longitudinal 'building-up' process is called polymerization, which usually results in the association of 13 parallel protofilaments to form a microtubule [22]. This renders a polarity due to different polymerization rates of the two ends, resulting in a faster-growing plus end and a slower-growing minus-end [4].

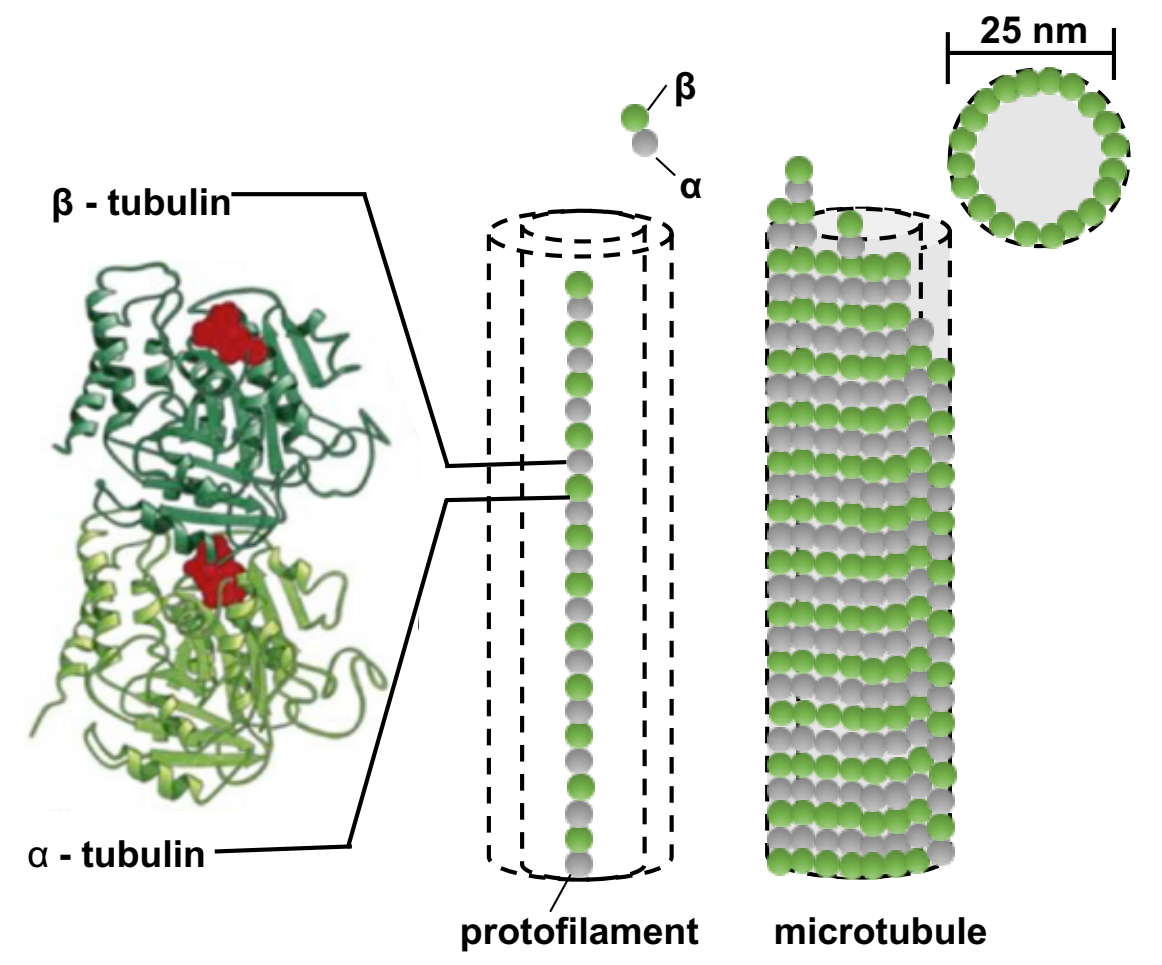

Figure 1.2: Molecular assembly of a microtubule, adapted from [3]

MT polymerization occurs in the presence of magnesium ions and a nucleotide 
GTP, which binds to $\beta$-tubulin and gets hydrolyzed, while $\alpha$-tubulin also binds GTP in a non-hydrolyzable manner. These reversible binding and hydrolysis cycles cause MTs to switch constantly between growth and shrinking. During polymerization, GTP bound to the $\beta$-tubulin gets hydrolyzed to GDP, which results in destabilization and subsequent depolymerization of the microtubule, a process called 'catastrophe'. GTP bearing dimers form a cap on the filament and can prevent catastrophe from occurring, in a process termed as 'rescue'. This constant and rapid switch between the phases of growth and shrinkage is termed as the 'dynamic instability' of MTs [67]. Hence, fundamental cellular processes of locomotion, morphogenesis, and reproduction rely on this ability of MTs to change their organization.

As MTs are biopolymers characterized by a bending stiffness, they can be envisioned to be similar to isotropic rods that are continuously flexible. This approximation is based on the worm-like chain (WLC) model for bending polymers [62, 86]. The WLC model can be used to characterize a filament's resistance to bending under thermal fluctuations to determine its persistence length.

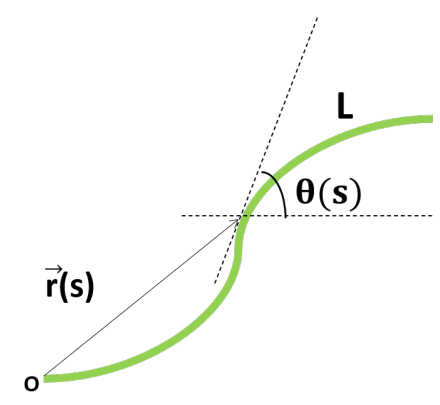

Figure 1.3: Modelling a filament as a continuous string with a bending stiffness, where $\vec{r}(\mathrm{~s})$ is the position vector along the length of a filament $\mathrm{L}$ and $\theta(\mathrm{s})$ is the angle between the tangent vector and a chosen reference axis

\subsection{Motor proteins : The molecular nanomachines}

Motile activities that occur within cells are predominantly due to linear motor proteins that move on actin and MTs. Motor proteins are unique chemo-mechanical enzymes that convert chemical energy derived from ATP hydrolysis to mechanical work directly. They physically walk along the length of filaments and generate forces to move cargoes along filaments that serve as tracks.

Cytoskeletal motor proteins belong to three main families : kinesin and dynein (that move along MTs) and myosin (that moves along actin filaments). Over the years, the advancement of various genome projects has resulted in an expanding number of motor sub-families : eighteen sub-classes of myosin, fourteen of kinesins 
and three of dyneins [15, 45]. Myosin is responsible for muscle contraction, cell division and its interaction with the actin cytoskeleton causes cell crawling on surfaces. Meanwhile, kinesin (+ end-directed) and dynein (- end-directed) are responsible for generating forces on MTs that act as polar pathways for intracellular communication [45]. On a broader scale, they are involved in directed transport, chromosomal segregation, flagellar and ciliary beating. The framework for the interpretation of motor motility along their associated filaments is based on their latching mechanism, subsequent ATPase cycle and resulting conformational changes.

In the early 1990s, the importance of motor proteins as cytoskeletal re-organizers that crosslink filaments and cause their sliding was revealed. While new families of motors were being discovered, subsequent studies dealt with the processivity of single motors in terms of their run lengths and association times. Regarding single kinesin behaviour, the first work by Ronald Vale and co-workers [102] revealed a direct TIRF visualization of single fluorescently-labelled kinesin on surface-adsorbed axonemes. Further, ideas on motor number, binding and unbinding mechanisms, interaction stoichiometry [103] as well as MT polarization were obtained [48, 27, 47].

We shall now deal with structural composition and action mechanism of kinesin, the motor protein that is the focus of study in this work.

\subsubsection{Kinesin-1 : Structure and stepping mechanism}

Kinesin-1 (kinesin) was discovered in 1985 as a heterotetramer (120 kDa), composed of two identical heavy chain polypeptides that contain an N-terminal globular motor domain which has MT-binding properties [31]. These heavy chains dimerize to form a parallel coiled-coil stalk. The stalk is connected to a C-terminal domain that interacts with the kinesin-light chains for cargo-binding as seen in Figure 1.4 [21]. When there is no cargo to bind, the C-terminal domain regulates kinesin's catalytic activity by folding back into the motor domain.

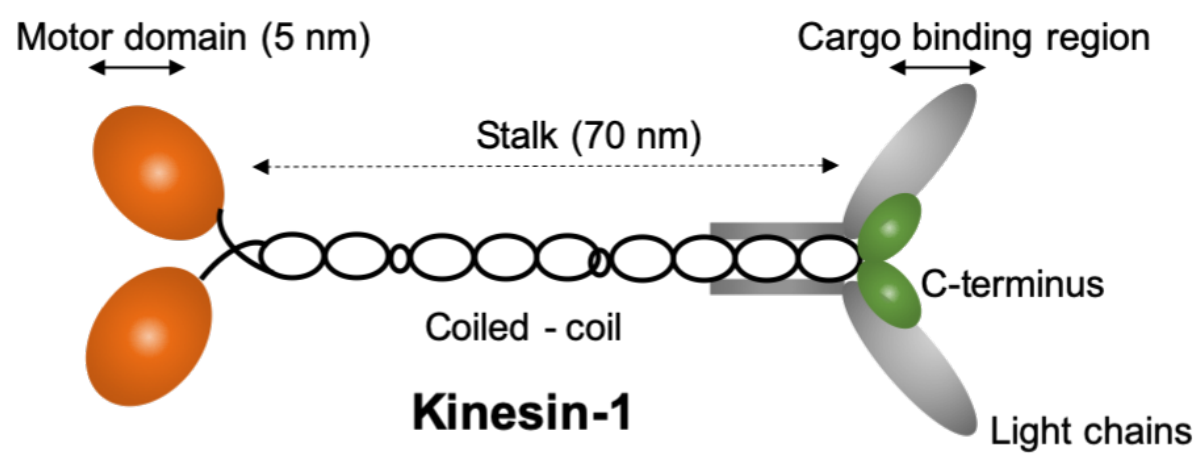

Figure 1.4: Schematic representations of the structure of kinesin-1 and its associated chains, adapted from [31]. 


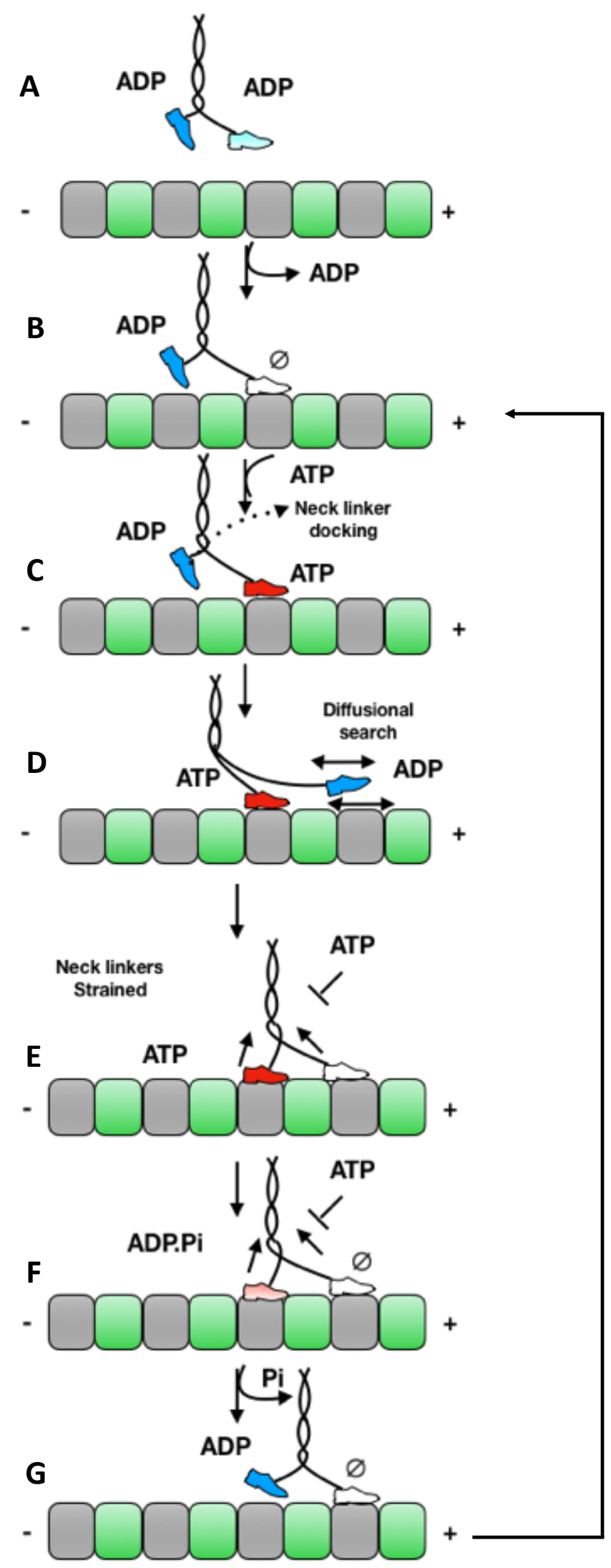

Figure 1.5: Schematic representation of the stepping mechanism of Kinesin-1, adapted from [21].

The stepping mechanism of conventional kinesin can be explained through a 
series of steps shown in Figure 1.5. Each identical kinesin head contains a tightly bound ADP. The heads establish coordination with each other when the first head collides with the MT, resulting in a rapid ADP release [40] at a dissociation rate of $200 \mathrm{~s}^{-1}$. At the leading head, ATP binding promotes the necker link docking onto the catalytic core. This leads to a rapid forward movement of the trailing, tethered head towards the plus end of the MT, followed by the next MT binding site (Steps C-E). The tethered head now moves forward to the next binding site at a rate of at least 10,000 per s because of the high local tubulin concentration at the MT lattice. The advancing head can find the correct $\beta$-tubulin binding site due to the role played by Brownian motion. This is because the neck linker docking alone cannot be sufficient to account for the entire $8 \mathrm{~nm}$ displacement.

Once the tethered head is attracted to the MT, the second ADP is released. This forms an intermediate (as seen in step E) with both heads tightly bound to the MT and the neck linkers get strained. The progression from the step E is controlled to prevent premature ATP binding and hydrolysis at the leading head. A lack of controlling at this step would otherwise result in the disruption of coordination and a complete detachment of the motor. Usually, ATP hydrolysis that occurs at the rear-end head is a fast step (120 per s) that precludes the rate-limiting phosphate release at 50 per s. The ADP state results in a weak binding to the MT surface (Step $\mathrm{G})$, biasing the rear-end head for detachment. The Step G intermediate has a higher probability for ATP binding, and the cycle is repeated, back to step B. However, the two motorheads have now changed their positions from the initial step, and the kinesin-1 has already stepped ahead by $8 \mathrm{~nm}$ along the MT-lattice with one ATP turnover for every $8 \mathrm{~nm}$ step.

In this work, we make use of a truncated kinesin protein, K401-BIO-6xHis purified from a Drosophila heavy chain kinesin-1, truncated at the residue K401 and modified to contain a 6 -histidine sequence [96]. We use it in the form of kinesin clusters through the four biotin-binding sites of streptavidin. The kinesin clusters induce sliding between a pair of oppositely-oriented MTs.

We study their behaviour as synthetic active matter systems that can be used to reconstitute ciliary behavior.

\subsection{Synthetic active matter systems}

We use synthetically assembled MTs and kinesin-1 mixtures as the basic building blocks of our research. Their self-organizing behaviour reveals that they are internally driven active matter systems.

The first model systems employed to study single kinesin behaviour were gliding 
and stepping assays as illustrated in Figure 1.6(A) and (B). Gliding assays are characterized by filaments gliding over an assembly of surface-immobilized motors. In stepping assays, the filaments are laid out on the surface and become tracks for the motors to move along [93, 92, 102]. Similar to the cytoskeleton, the synthetic materials used are driven out-of-equilibrium by the same energy-consuming processes: GTP-fuelled cycles of polymerization/depolymerization and ATP-fuelled stepping of motor proteins 73 .

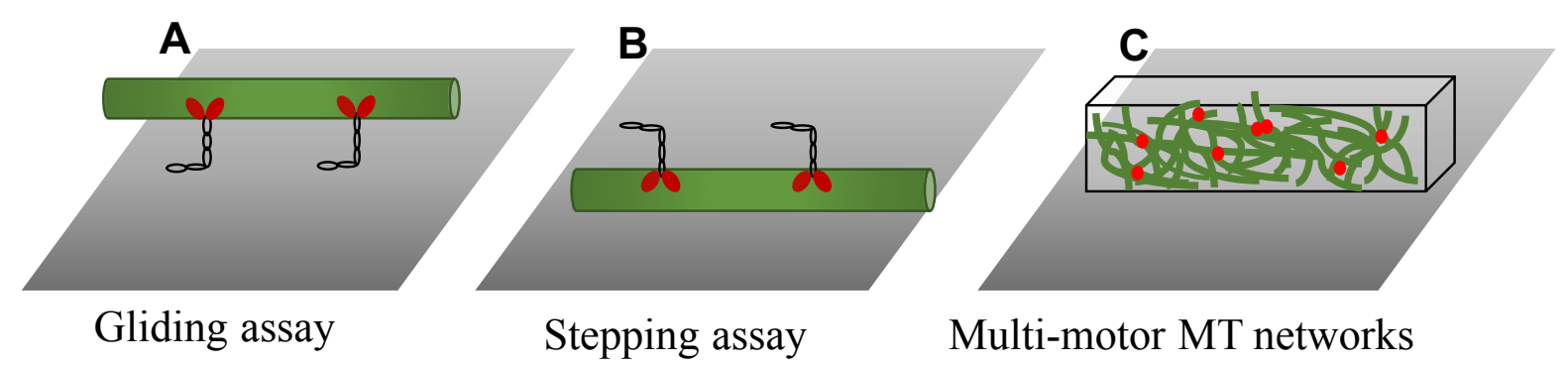

Figure 1.6: A comparison of the (A) gliding and (B) stepping of single kinesins to (C) motor cluster-MT networks that we studied.

The works of Nedelec et al [71, 72, 97] revealed that mixtures of MTs with motor protein clusters exhibit anomalous structures such as radial asters similar to the mitotic spindle. Experimental designs have been modified to include nonadsorbing polymers to induce MT bundling [74, 44] that enhances the interfilament sliding (Figure 1.7). These generate extensile networks with active stresses, thus comprising a new class of bulk active materials [88, 42]. To induce activity through motor proteins in such systems, the MTs are subjected to bundling through the addition of a polymer such as PEG. This is to reconstitute nexin links that connect MT doublets and hence, translate motor protein sliding into bending in natural cilia.

A more recent terminology to the motion observed in such active matter experiments is active turbulence or mesoscale turbulence [106]. As a high density of active particles move and interact mechanically and hydrodynamically, the growing strength of particle interactions are characterized by regions of high vorticity. The vortices are found to be on a length scale of $\sim$ ten times that of an individual particle [20]. In this field, active extensile bundles of MTs are considered to be versatile building blocks due to the high processivity of motor clusters in mixed polarities. The extensile networks are mainly driven by the self-extension, buckling, fracturing and re-annealing of their constituent MT bundles. When active bundles were sedimented onto a surfactant-stabilized oil/water interface, they formed dense quasi-2D films where MT bundles align as an extensile nematic [88, 56]. Studies 


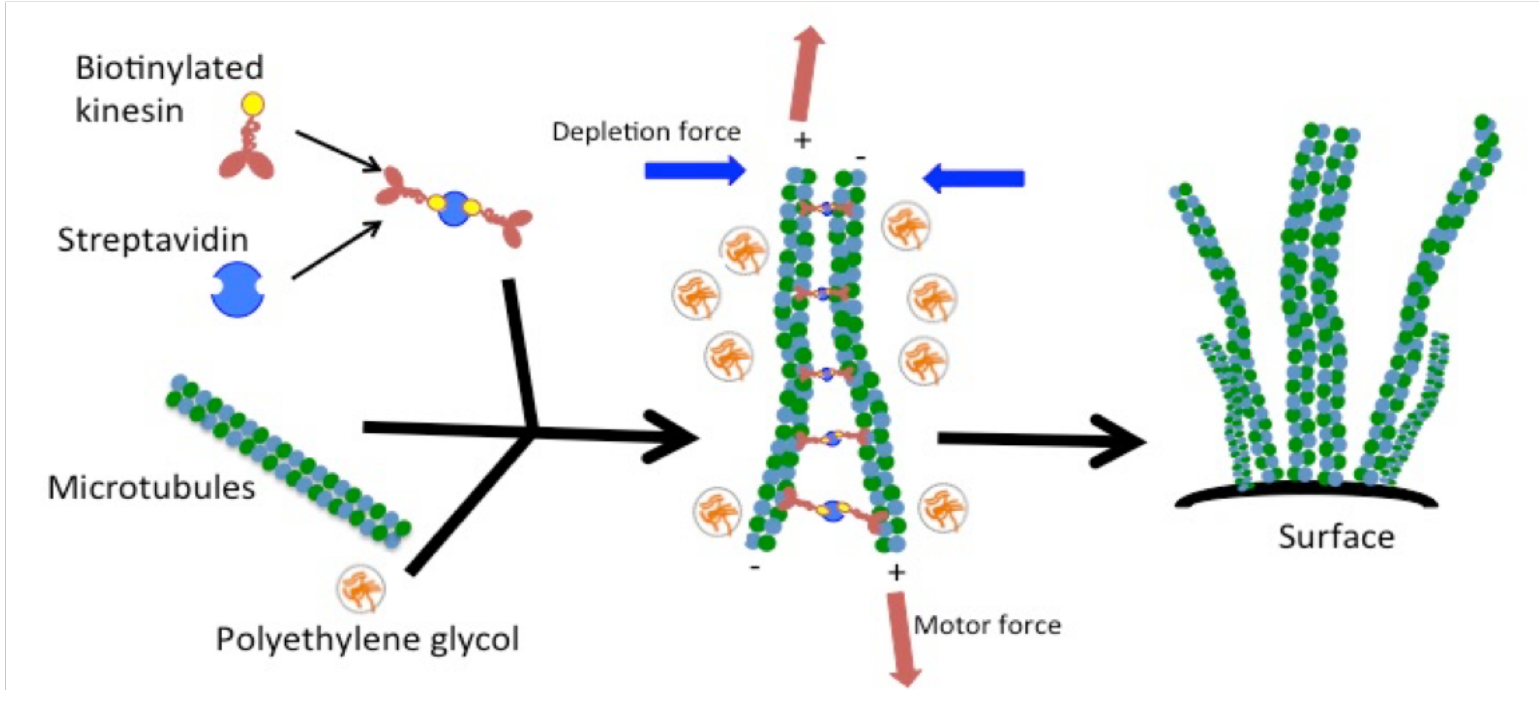

Figure 1.7: Schematic representation of the minimal building blocks, modified from [89].

have revealed that the resulting active stresses can continuously generate topological defects through bending instabilities. They exhibited chaotic dynamics and the defects streamed through until annihilated by oppositely-charged defects [34, 33, 30]. As a result, they were found to gain an orientational order, resulting in an active nematic symmetry. More recently, active liquid crystals have exhibited alignment due to a uniform magnetic field, transitioning between turbulent and laminar flow regimes [37].

Why is it essential to study synthetic active matter systems? Self-organization plays an extensive role in the cytoskeleton. As seen in ciliary beating and cytoplasmic streaming, motor proteins are the active components that bring the system out of equilibrium. These biological processes served as an inspiration for this work as there is still no clear description of their underlying mechanisms.

In this work, we explore the behaviour of MT-kinesin configurations at hierarchical levels ranging from dense active networks to active bundles and filaments. We observe the interesting behaviours of bundled active networks under geometrical confinement, that leads to a range of behavior from a nematic ordered phase to an isotropic phase. In turn, we study the self-assembly and interactions between MTs and motor proteins towards their applications in biomimetic structures. 


\section{Chapter 2}

\section{Materials and Methods}

This section explains all the experimental details employed throughout the thesis. We start from the basics of MT polymerization in vitro and proceed to the inclusion of motor proteins to create active MT networks. This chapter shall also delve into the important functions of the various building blocks employed. Additionally, we explore microfluidic and micropatterning methods performed for surface attachment of filaments. We conclude with brief explanations of the various computational methods employed for experimental analysis.

\subsection{Polymerization of microtubules}

MT polymerization is the process of assembing MTs through the lateral association of tubulin monomers in vitro. It is carried out using tubulin subunits that polymerize end-to-end in the presence of GTP in phases of nucleation and elongation.

We obtained Porcine brain tubulin commercially as a lyophilized powder that was reconstituted to a $10 \mathrm{mg} / \mathrm{ml}$ stock inside an M2B buffer (M2B : $80 \mathrm{mM}$ PIPES, adjusted to a $\mathrm{pH}$ of 6.8 using $\mathrm{KOH}, 1 \mathrm{mM}$ EGTA, $2 \mathrm{mM} \mathrm{MgCl}_{2}$ ). The aliquots of reconstituted tubulin were snap-frozen in liquid nitrogen and stored at $-80{ }^{\circ} \mathrm{C}$. To grow MTs, we make use of $2.7 \mathrm{mg} / \mathrm{ml}$ Hilyte $^{T M} 488$ labelled porcine brain tubulin (Cytoskeleton, inc., U.S.A.) inside an M2B buffer along with $4 \mathrm{mM} \mathrm{MgCl}_{2}, 5 \%$ DMSO and 1.5 mM GTP to promote MT assembly [19, 67]. The difference between M2B and traditionally used buffers such as BRB80 (Brinkley Reassembly buffer) is that it contains $2 \mathrm{mM} \mathrm{MgCl}_{2}$ in comparison to $1 \mathrm{mM}$ present in BRB80.

Tubulin is a labile molecule sensitive to temperature and hydrostatic pressure that changes into a non-polymerizable state within a few minutes. Hence, the entire process of pipetting and mixing is done on ice within $10 \mathrm{~min}$ and the tubulin is added as the last step. The prepared microtubule mixture is immediately incubated in an oven at $37^{\circ} \mathrm{C}$ for $30-45$ min depending on the experiment. Longer incubation 
times result in longer microtubules. 5 min before completion of the incubation period, fresh taxol ( $1 \mathrm{mM}$ in DMSO) from $-20{ }^{\circ} \mathrm{C}$ is thawed at room temperature, quickly vortexed and diluted in $\mathrm{M} 2 \mathrm{~B}$ to a final concentration of $10 \mu \mathrm{M}$. At the end of the polymerization, the MT mixture is immediately diluted $\sim 13$ fold with the taxol-M2B solution. Adding taxol is an important step as it arrests polymerized MTs from undergoing dynamic instability that would otherwise result in a quick diminishing of its length. At this concentration, the MTs appear as dense carpets and can be better visualized by diluting, for example, 10, 20 and 50 fold in $10 \mu \mathrm{M}$ taxol in M2B. The average length for taxol-stabilized MTs polymerized for $30 \mathrm{~min}$ was found to be $19 \pm 10 \mu \mathrm{m}$ as shown in Figure 2.1.
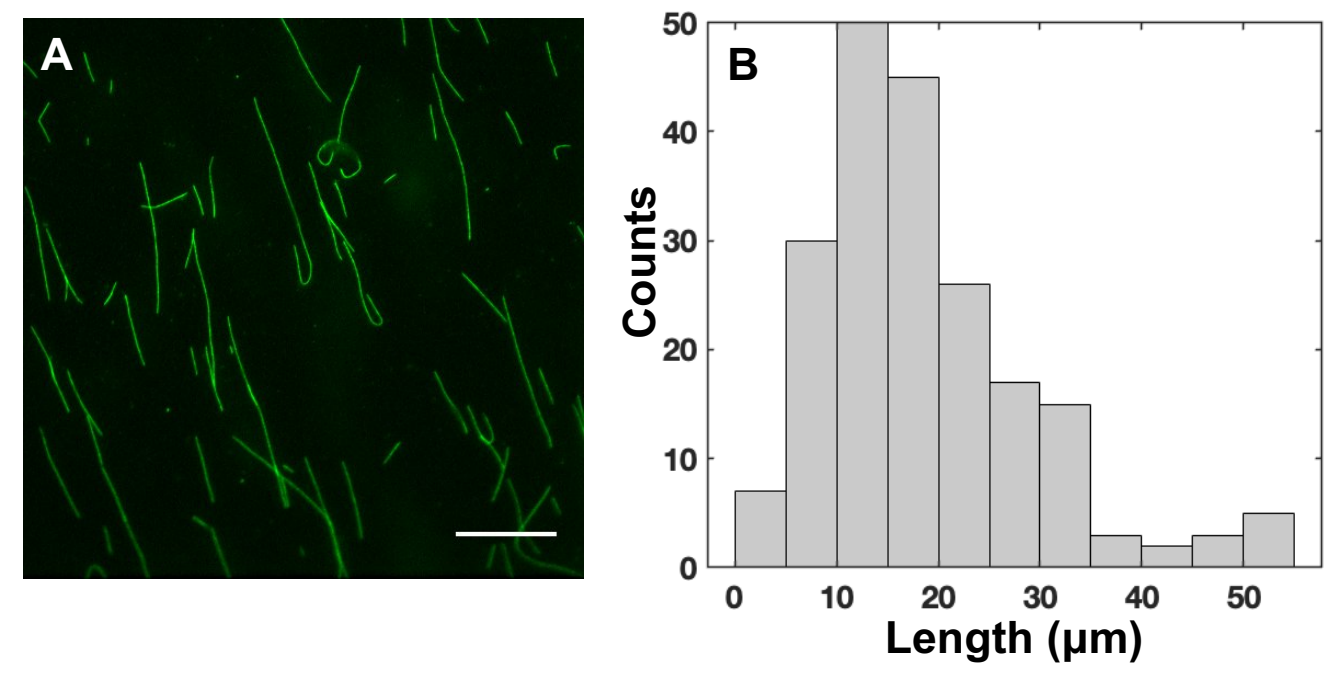

Figure 2.1: (A) Exemplary image showing polymerized microtubules adsorbed on a cleaned glass surface. (B) Length distribution of 50-fold diluted microtubules. Scale bar : $50 \mu \mathrm{m}$

\subsubsection{Confocal Laser Scanning Microscopy}

The imaging setup used in this work is an Olympus FluoView 1000 confocal microscope (Olympus, Japan). Confocal Laser Scanning Microscopy utilizes a pinhole in the optical path of its excitation laser. This pinhole aperture reflects the incoming laser beam via a dichromatic mirror onto the objective, focussing the light onto a single spot on the specimen. The beam moves along the area of the sample in a raster pattern, and this method is known as point scanning. Excited fluorophores cause photons to be emitted at different wavelengths that are focused on the photomultiplier, excluding signals from above and below the focal plane [69]. The combination of point scanning and use of pinhole as a spatial filter contribute to the formation of a confocal image. Series image acquisitions were performed with a Photometrics 
Cascade II EMCCD camera. The objectives used were usually a $20 \times$ and a $63 \times$ oil-immersion objective (Olympus, Japan) depending on experimental requirements.

For some experiments mentioned, we additionally use an epifluorescence microscopy setup. It is an integrated system consisting of a fluorescence microscope, multiple laser light sources and a confocal scan head containing the lens system. It consists of an Olympus IX81 inverted fluorescence microscope (Olympus, Japan) that excites samples using a Lumen 200 metal arc lamp (Prior Scientic Instruments, U.S.A.).

Typically, the sample imaging chambers for experiments are fabricated by using a double tape of thicknesses 10-100 $\mu \mathrm{m}$ (Nitto Denko Corporation, Japan) sandwiched between two glass coverslips $\left(24 \times 60 \mathrm{~mm}^{2}\right.$, VWR $)$. As most of the experiments require tightly sealed chambers to avoid fluid streaming, a window of size $8 \times 8 \mathrm{~mm}$ was cut on the spacer and completely sealed after equivalent volumes of samples were pipetted onto the glass surface.

\subsection{Depletion agents induce MT bundling}

MT filaments can be merged to form bundles through the use of a depletion agent. The non-adsorbing polymers that are known to induce such depletion interactions between MTs are poly(ethylene glycol) or PEG, dextran and methylcellulose [50]. The concept of the depletion effect (or macromolecular crowding) is based on the increasing total volume available for the depletant polymers between at least two filaments. The depletant polymers (such as PEG) can be represented as spheres whose radius is called the polymer's radius of gyration, $\mathrm{R}_{g}$ (Figure 2.2). But, the volume occupied by the MTs themselves is not available to the polymers. Due to this, the centre of mass of each PEG polymer can get no closer than its radius of gyration to the surface of an MT. Every MT gets surrounded by an external cylindrical shell, resulting in an 'excluded volume' that is unavailable for the polymers to pass through. By reducing this excluded volume, the space available for the PEG molecules increases. This causes a net increase in entropy due to the presence of many more depletant polymers than MTs.

Mathematically, the MT filaments reduce the volume of space available to the PEG chains from $\mathrm{V}$, the entire space volume to $V-V_{e x}$. If $N$ is the number of PEG molecules and $\nu$ is a constant with units of volume, denoting the volume of a unit cell in a lattice model. The change in free energy due to this excluded volume is given by :

$$
G_{e x}=-N k_{B} T \ln \left(\frac{V-V_{e x}}{\nu}\right)+N k_{B} T \ln \left(\frac{V}{\nu}\right)
$$

Assuming that the excluded volume $V_{e x}<<V$ and approximating $\ln (1+x) \approx x$, 


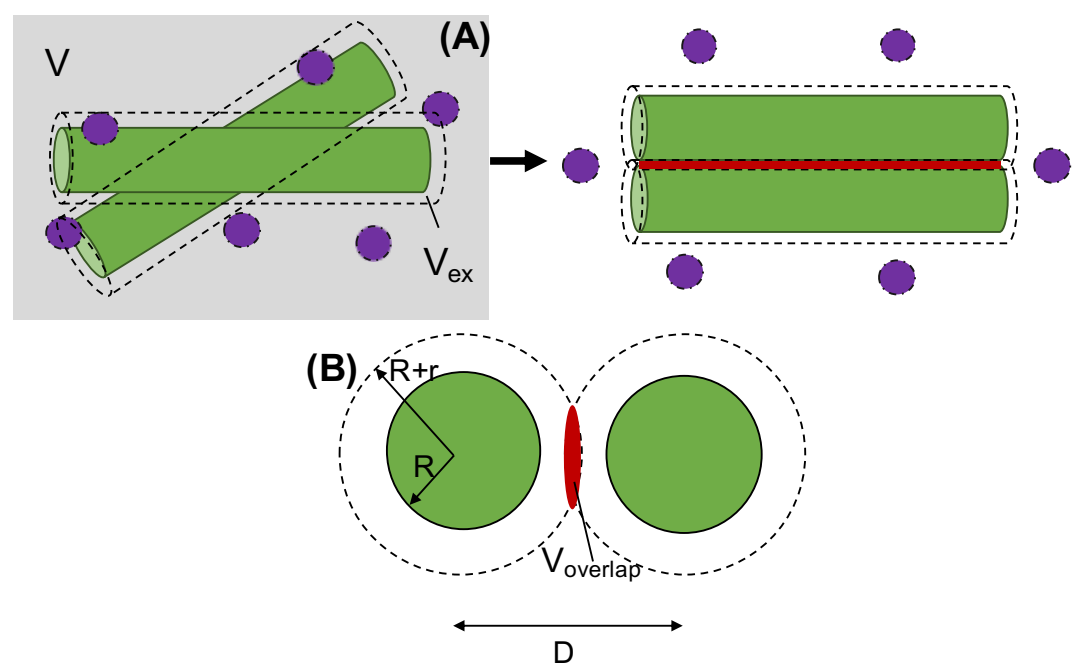

Figure 2.2: (A) Large rod-like molecules (in green) in volume $\mathrm{V}$ with smaller particles (in purple). $V_{e x}$ is the excluded volume i.e. the volume of the shell larger than the bare volume of the rods. (B) The depletion force, $F_{d}>0$ when 2 rods come within a distance $D<2(R+r)$, determined by the overlap volume.

we get :

$$
G_{e x}=N k_{B} T\left(\frac{V_{e x}}{V}\right)
$$

When the $V_{e x}$ regions of 2 MTs overlap, the PEG polymers cannot go between the filaments. This polymer concentration difference causes an imbalance of osmotic pressure, resulting in an effective attraction between them. The magnitude of this depletion force is given by $\frac{d G}{d D}$, whose positive value indicates an attractive force [78].

When an MT bundle is formed, the depletion forces on the filaments remain constant until the overlap is maximized. The force scales linearly with the area of cross-section of the overlap, thus depending on the inter-MT distance between individual filaments within a bundle.

For experiments that include PEG as a depletant polymer, the strength and the range of the depletion forces can be tuned with the polymer size and concentration respectively [44]. For our experiments, we make use of PEG of molecular weight $20 \mathrm{kDa}$ at final concentrations between 0.6 - 1\% within the polymerization mix. Likewise, in cases of diluting final MT mixtures after incubation, an equivalent volume of PEG is added along with taxol to conserve the final concentration.

Previous X-ray scattering studies [75, 74, 44] of PEG-depleted MT bundles have revealed that PEG of molecular weight $20 \mathrm{kDa}$ may result in MT bundles having at least 4-6 merged MTs. 


\subsection{Creating active microtubule bundles}

As discussed in the introduction, we make use of truncated kinesin-1 complexes as activity-generating components. By polymerizing MT mixtures in the presence of the motors, we were able to generate dense active bundles.

The plasmid that codes biotin-labelled kinesin 401 (K401) was a gift from Jeff Gelles (pWC2 - Addgene plasmid \# 15960; http://n2t.net/addgene:15960;

RRID_Addgene_15960 ) [96] and was purified according to previously published protocols [32, 107]. The kinesin complexes are conjugated with MTs in the presence of various agents.

For experimental convenience, we mix several initial mixtures before incorporation into a final solution. Essentially, these are the (i) Kinesin-streptavidin complexes (ii) an active mixture that contains the motor proteins, energy-inducing ATP and associated ATP-regeneration components, depletion-inducing PEG and an anti-fade mixture and (iii) the MT polymerization mix [89]. The details of the components used are tabulated in Table 2.1.

We shall also briefly delve into the functions of the various components incorporated into the MT-motor protein systems. Firstly, the kinesin-streptavidin (K-S) complexes are prepared and incubated on ice for $15 \mathrm{~min}$ [71]. During this period, the biotin-labelled kinesin interacts non-covalently with tetrameric streptavidin to form multi-motor complexes. Simultaneously, we gather the components to form a final active mixture. These 'sub-mixtures' include an ATP regeneration system that contains PK/LDH enzymes which uses PEP as a fuel to recycle ADP back into ATP during hydrolysis [58]. Additionally, we prepare an oxygen-scavenging system that is known to effectively prevent the irreversible loss of fluorescence or photo-bleaching in our assay. It consists of D-glucose that gets oxidised to gluconic acid through glucose oxidase, decreasing oxygen in the solution. Hydrogen peroxide emerges as a by-product and is degraded by catalase in a second reaction step. The Trolox (6hydroxy-2,5,7,8-tetramethylchroman-2-carboxylic acid) and DTT delay the bleaching further through triplet-state quenching and maintain a protein-friendly reducing environment [2].

Once these mixtures are assembled, at the end of $15 \mathrm{~min}$, they are combined with the K-S complex, ATP and PEG. The microtubule bundle polymerization mix is prepared as previously stated using concentrations tabulated in Table 2.2. It is important to note that while ATP provides a chemical energy source for the movement of kinesin, an important cofactor of ATP hydrolysis is magnesium. Hence, the active microtubule bundles are polymerized in a high-concentration $\mathrm{MgCl}_{2}$ environment. Thus, $68 \mathrm{mM} \mathrm{MgCl}_{2}$ in $\mathrm{M} 2 \mathrm{~B}$ is used to prepare the taxol mixture. Finally, we combine the active mixture with the microtubule bundles polymerization mix 
CHAPTER 2. MATERIALS AND METHODS

\begin{tabular}{|c|c|c|}
\hline \multicolumn{3}{|c|}{ Kinesin-streptavidin complexes } \\
\hline Component & Concentration/volume & Supplier/preparation \\
\hline Kinesin-1 & $0.2 \mathrm{mg} / \mathrm{ml}$ & purified according to $[31,100]$ \\
\hline Streptavidin & $0.1 \mathrm{mg} / \mathrm{ml}$ & Sigma-Aldrich , S4762 \\
\hline Dithiothreitol (DTT) & $0.9 \mathrm{mM}$ & Sigma-Aldrich, D0632 \\
\hline M2B (buffer) & to fill to a final volume of $28 \mu \mathrm{l}$ & $\mathrm{pH}=6.8$, prepared as above \\
\hline \multicolumn{3}{|c|}{ Active mixture $=$ Active components + ATP regeneration system + Oxygen-scavenging system } \\
\hline \multicolumn{3}{|c|}{ Active components } \\
\hline Component & Concentration/volume & Supplier/preparation \\
\hline Kinesin-streptavidin(K-S) complex & $4 \mu 1$ & prepared as above \\
\hline Adenosine triphosphate (ATP) & $2 \mathrm{mM}$ & Sigma-Aldrich, A2383 \\
\hline Poly(ethylene glycol) 20,000 & $1 \%$ & Sigma-Aldrich, 81300 \\
\hline \multicolumn{3}{|c|}{ ATP regeneration system } \\
\hline Component & Concentration/volume & Supplier \\
\hline Phosphoenol pyruvate (PEP) & $32 \mathrm{mM}$ & Alfa Aesar, B20358 \\
\hline Pyruvate Kinase lactic & $1.7 \mu l$ & Sigma-Aldrich, P-0294 \\
\hline dehydrogenase enzyme (PK/LDH) & & \\
\hline \multicolumn{3}{|c|}{ Oxygen-scavenging/anti-fade system } \\
\hline Component & Concentration & Supplier \\
\hline D-Glucose & $0.5 \mathrm{mg} / \mathrm{ml}$ & Sigma-Aldrich, G7528 \\
\hline Glucose Oxidase & $0.2 \mathrm{mg} / \mathrm{ml}$ & Sigma-Aldrich, G2133 \\
\hline Catalase & $0.05 \mathrm{mg} / \mathrm{ml}$ & Sigma, C40 \\
\hline Trolox & $2.4 \mathrm{mM}$ & Sigma-Aldrich, 238813 \\
\hline Dithiothreitol (DTT) & $0.65 \mathrm{mM}$ & Sigma-Aldrich, D0632 \\
\hline \multicolumn{3}{|c|}{ Total volume of active mixture $=50 \mu \mathrm{l}$} \\
\hline
\end{tabular}

Table 2.1: Initial sub-mixtures to be combined with final MT bundle polymerization mixture

in the ratio $2: 1$ and then add the taxol- $\mathrm{MgCl}_{2}$ solution which now reaches a final concentration of $7.8 \mu \mathrm{M}$.

The mixture is incubated in the oven at $37^{\circ} \mathrm{C}$ for $45 \mathrm{~min}$, pipetted onto a tightly sealed glass chamber and visualized under the microscope.

Typically, the experiments illustrated above result in dense MT bundle carpets with kinesin clusters translocating across them. For some experiments that will be dealt with in Chapter 3, we labelled motor clusters through the streptavidin tag. Hence, in place of unlabelled streptavidin, $0.1 \mathrm{mg} / \mathrm{ml}$ Cy-3 labelled Streptavidin (Sigma, S6402) was used to create the kinesin complexes and the remaining mixtures were prepared as usual. 


\begin{tabular}{|c|c|c|}
\hline \multicolumn{3}{|c|}{ Microtubule bundles } \\
\hline Component & Concentration & Supplier \\
\hline $\begin{array}{c}\text { Hilyte 488 labelled porcine } \\
\text { brain tubulin }\end{array}$ & $2.7 \mathrm{mg} / \mathrm{ml}$ & $\begin{array}{c}\text { Cytoskeleton inc., } \\
\text { Denver, CO }\end{array}$ \\
\hline $\begin{array}{c}\left.\text { Magnesium chloride ( } \mathrm{MgCl}_{2}\right) \\
\text { Dimethyl sulfoxide (DMSO) }\end{array}$ & $4 \mathrm{mM}$ & Sigma-Aldrich, M8266 \\
\hline $\begin{array}{c}\text { Guanosine 5'-triphosphate } \\
(\mathrm{GTP})\end{array}$ & $1 \mathrm{mM}$ & Sigma-Aldrich, D8418 \\
\hline $\begin{array}{c}\text { Poly(ethylene glycol) } 20,000 \\
\text { M2B containing } \mathrm{MgCl}_{2}\end{array}$ & $\begin{array}{c}68 \mathrm{mM}\left(\mathrm{MgCl}_{2}\right), \\
\text { down to } 7.8 \mathrm{mM}\end{array}$ & prepared as above \\
\hline Paclitaxel (Taxol) & $7 \mu \mathrm{M}$ & Sigma-Aldrich, T7402 \\
\hline
\end{tabular}

Table 2.2: The MT bundle mix that contains PEG and $\mathrm{MgCl}_{2}$ is combined with the active mixture as a final step.

\subsubsection{Individual MT-motor protein bundles}

To visualize individual active MT bundles, we performed the standard experiment and at the end of the incubation time, diluted a part of the mixture 20-fold to lower the density of MT bundles. The dilution mix contains ATP, PEP and PK, PEG and taxol in M2B added in quantities sufficient to conserve the final concentrations (Refer Table 2.1 and Table 2.2).

\subsection{A modified gliding assay}

To study the influence of a few randomly distributed motor clusters' behaviours on a single MT, we implemented a modified gliding assay. It makes use of a PLL-PEG coated surface that contains a few MTs pinned on one end (Refer Section 2.6.1). Kinesin-decorated MTs can be obtained by preparing the MT polymerization mix and an active mixture separately. We do not include PEG as we intend to study individual filament behaviour, rather than bundles. Once the MTs are incubated for 30 min at $37^{\circ} \mathrm{C}$, they are diluted 2000 -fold in M2B that contains taxol. They are further combined with the active mixture in different ratios depending on intended experiments. 


\subsection{Networks under geometrical confinement}

A significant part of this work deals with observations of MT-motor protein networks under confinement in chambers of different heights, namely $10 \mu \mathrm{m}$ and $100 \mu \mathrm{m}$. Additionally, we employ two polymerization modes/environments for these MT-motor protein networks i.e the standard polymerization in an Eppendorf tube followed by visualization in a chamber and polymerization within a sample chamber directly. The 4 cases that are thus generated for our study are summarized in Figure 2.3. As discussed above, direct polymerization of MT bundles in the presence of motors results in dense networks. To track the movement of individual bundles (as a result of motor protein cluster action), we employed passive tracer particles in the form of fluorescent carboxylated beads. When these tracers are incorporated into the networks, their trajectories can be tracked as a measure of the movement of individual MT bundles.

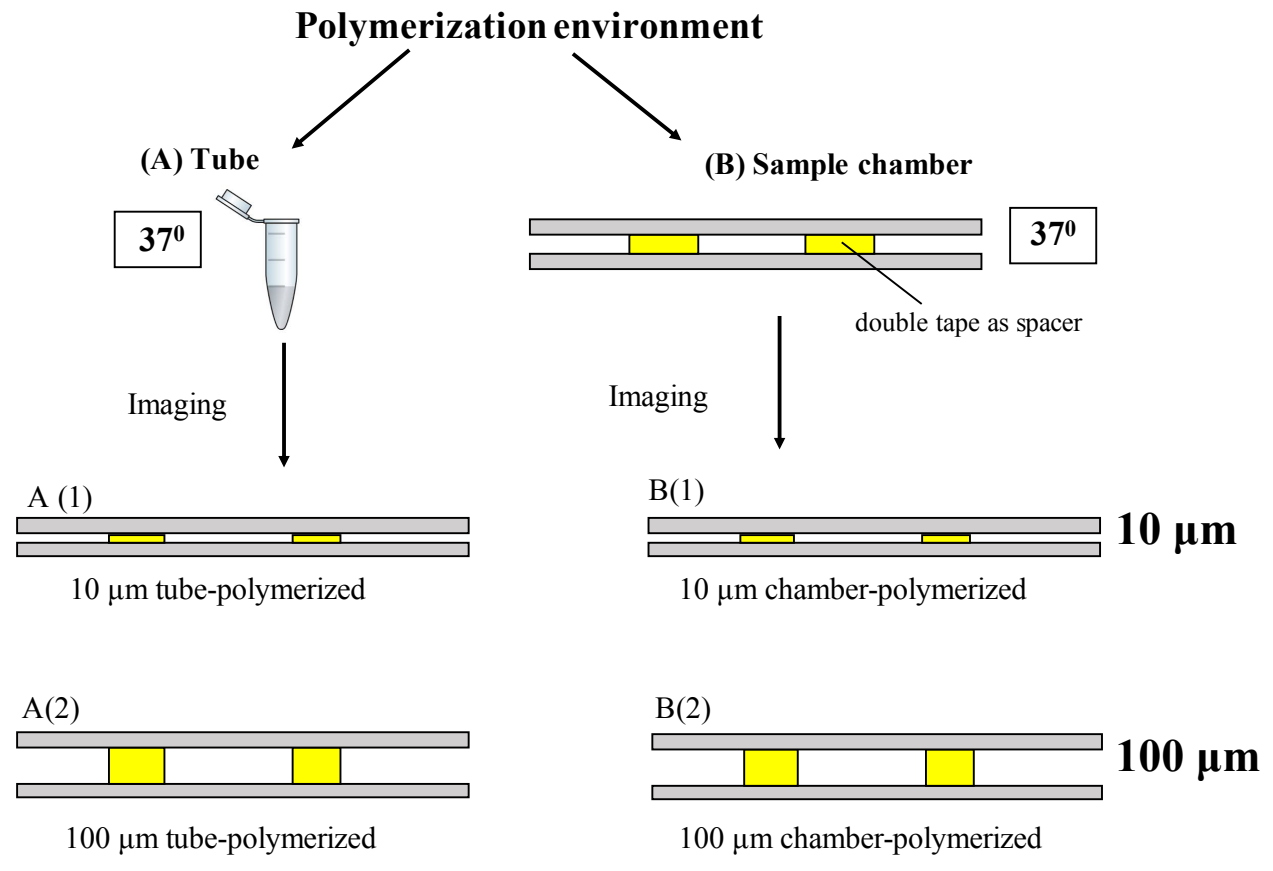

Figure 2.3: Schematic of the experimental variations in sample chambers of 2 different heights.

The experimental procedure remains the same as described in section 2.3, with the additional incorporation of the tracers:

1. We make use of red fluorescent carboxylate-modified microspheres of diameter $2 \mu \mathrm{m}$ ( Fluospheres, Invitrogen). Firstly, the bead stock is diluted 8 times in $10 \mathrm{mM}$ HEPES at $\mathrm{pH}$ 7.4. The beads are further spun for $1 \mathrm{~min}$ at $4000 \mathrm{rpm}$ two times and resuspended in 8 times HEPES. 
2. The standard procedure of creating active MT bundles is performed by modifying the concentration of ATP and PEG to $1 \mathrm{mM}$ and $0.6 \%$ respectively.

3. At the end of the mixing procedure, the bead solution is incorporated along with the $\mathrm{MgCl}_{2}$-taxol solution to a final volume of $4 \%(\sim 200 \mathrm{X}$ diluted).

4. The final mix is incubated at $37^{\circ} \mathrm{C}$ for $30 \mathrm{~min}$. Samples of different polymerization environments are prepared at the same time. For example, a $10 \mu \mathrm{m}$ tube-polymerized and chamber-polymerized sample can be studied simultaneously to ensure the robustness of the experimental procedure.

\subsection{Surface passivation techniques}

A common obstacle associated with protein-based assays is the undesired nonspecific adsorption of proteins on surfaces. To counter this issue, surface passivation techniques are widely used to alter the interfacial energy at the protein-surface boundaries [25]. While earlier methods involved using non-covalent blocking proteins such as BSA (Bovine Serum Albumin) and casein, they are found to be inefficient in the case of motor protein experiments. Instead, we make use of PEG-based copolymers that are efficient protein repellants due to reduced charge-mediated and hydrophobic interactions [85]. In this work, we use 2 types of PEG copolymers :

- Poly (L-lysine)-g-poly(ethylene glycol) or PLL-PEG ('g' denotes a grafting ratio of 3.5 )

- methoxy-poly(ethylene glycol) silane or mPEG-Silane

The PLL-g-PEG (g : graft) copolymers rely on electrostatic interactions for selfassembly. The positively charged poly-L-lysine (PLL) gets attracted to the negatively charged glass coverslips and the charge-neutral PEG chains extend into the solution as illustrated in Figure 2.4. For the MT-motor protein experiments, we use a uniform PLL-PEG coating and the passivation steps shall be explained in detail below [51, 26]. Firstly, before any surface treatment, it is essential to perform a rigorous cleaning procedure of the glass surface to ensure complete removal of any dust traces that may interfere with the PLL-PEG coating or polymerization proteins later on.

1. Glass coverslips $\left(24 \times 60 \mathrm{~mm}^{2}\right.$, VWR $)$ are washed in $100 \%$ ethanol and wiped with clean absorbent paper. Following this, the dried coverslips are rinsed with de-ionized (DI) water and dried with a filtered airflow.

2. The slides are loaded in a suitable slide holder/box and sonicated in acetone for $30 \mathrm{~min}$ at room temperature. 


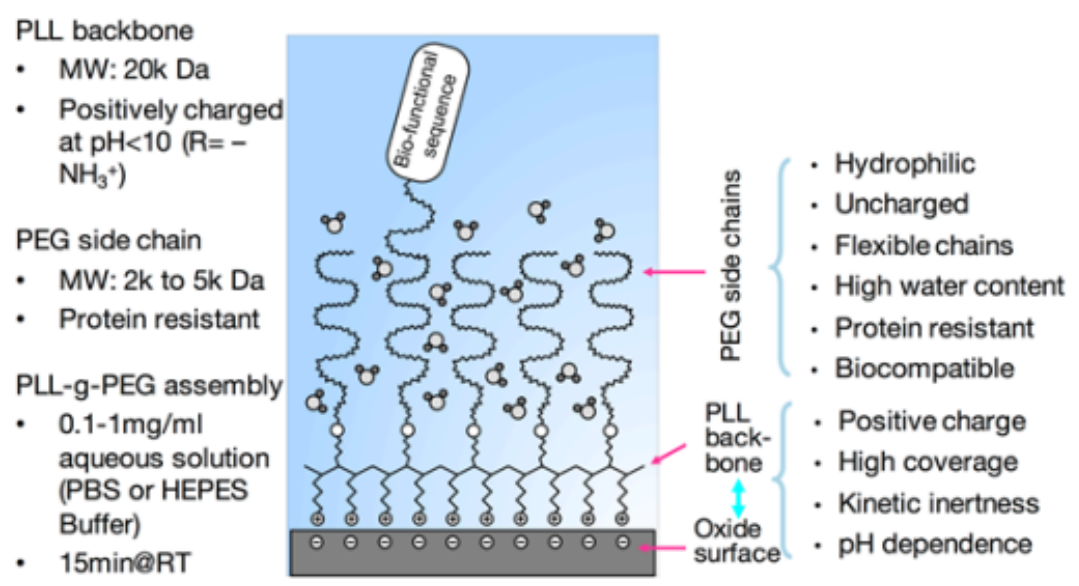

Figure 2.4: Schematic diagram depicting the function of PLL-PEG on a glass substrate. The image was taken from the SuSoS website.

3. The slides are transferred to a $96 \%$ ethanol solution and incubated for $10 \mathrm{~min}$ at room temperature, followed by extensive washing in DI water.

4. Now, the slides are incubated in a $2 \%$ Hellmanex III solution (Hellma Analytics) for $2 \mathrm{~h}$ at room temperature. Hereafter, they are once again washed extensively washed in DI water, dried under filtered airflow and stored in a sealed container.

5. The cleaned slides are activated in $\mathrm{O}_{2}$ plasma briefly for $30 \mathrm{~s}$ at 0.5 mbar (FEMTO, Diener Electronics, Germany). Immediately afterwards, the activated slides should be incubated in PLL-PEG.

\subsubsection{Coating PLL-PEG on glass coverslips}

1. The PLL-PEG stock (SuSoS AG, Switzerland) is diluted to $1 \mathrm{mg} / \mathrm{ml}$ in 10 mM HEPES buffer, (4-(2-hydroxyethyl)-1-piperazineethanesulfonic acid) at $\mathrm{pH}$ 7.4. Just before use, the solution is freshly diluted to $0.1 \mathrm{mg} / \mathrm{ml}$ once again in HEPES $10 \mathrm{mM}$, pH 7.4.

2. The glass coverslips from Step 5 of the cleaning procedure that is now oxidized are incubated with $200-500 \mu \mathrm{l}$ of $0.1 \mathrm{mg} / \mathrm{ml}$ PLL-PEG solution on Parafilm for $2 \mathrm{~h}$ at room temperature.

3. After $2 \mathrm{~h}$, an additional $200 \mu \mathrm{l}$ of HEPES is introduced between glass and parafilm. The coverslip is slowly lifted to allow complete removal of the PLLPEG solution. If the coating procedure was successful, the coverslip comes off perfectly dry with a tiny droplet remaining at the edge, which can be carefully 
wiped off with a Kimwipe (Kimtech Science). The PLL-PEG coated surfaces can be sealed and stored at $4{ }^{\circ} \mathrm{C}$ for up to 2 weeks.

\subsubsection{Coating silane-PEG on glass coverslips}

While PLL-PEG coatings on glass exhibit excellent surface passivation, silane-PEG coatings are considered better candidates to prevent non-specific adsorption of tubulin on surfaces [79]. Coating silane-PEG involves the following steps:

1. A $1 \mathrm{mg} / \mathrm{ml} \mathrm{mPEG-silane} \mathrm{solution} \mathrm{(Creative} \mathrm{PEGWorks,} \mathrm{MW}: 5 \mathrm{k}$ ) is made in $96 \%$ ethanol with $0.1 \%(v / v) \mathrm{HCl}$. The solution is warmed up to $50{ }^{\circ} \mathrm{C}$ under the bench, it is stirred to solubilize the silane-PEG.

2. Cleaned activated glass surfaces prepared above are incubated immediately in the silane-PEG solution at room temperature for $72 \mathrm{~h}$ with gentle agitation every few hours.

3. After this period, the coverslips are washed $3 \times$ in $96 \%$ ethanol and $3 \times$ in DI water successively.

4. The silane-PEG coated coverslips are further dried with pressurized filtered air and stored in a sealed container away from light at $4{ }^{\circ} \mathrm{C}$.

For most of the experimental results explained further, the PLL-PEG coating method was considered the optimum choice due to its robustness, except for micropatterning experiments discussed in the next section.

\subsection{Surface attachment via nucleation templates}

This section describes the experimental techniques that were employed to attach single MT filaments (in the absence of any motors) on surfaces (2D and 3D) by using two nucleation templates, namely

- MAP-tubulin

- Biotin-neutravidin-tubulin

These nucleation templates were incorporated into a microfluidic and micropattern setup respectively, the details of which are discussed below. 


\subsubsection{Microseeds as nucleation initiators}

The concept of attaching MTs to a suitable surface involves initiating preliminary nucleation via molecular intermediates, known as microseeds or seeds [67]. The seeds can be defined as MTs of length $0.5-2 \mu \mathrm{m}$ that attach to the nucleation templates and initiate further MT polymerization from their points of attachment. For seed polymerization, we make use of GMPCPP, a more stable, non-hydrolyzable analogue of GTP. The components to prepare seeds are tabulated in Table 2.3. Seed polymerization mixes are prepared on ice and can be snap frozen at $-80{ }^{\circ} \mathrm{C}$. When they are to be used in experiments, the polymerization aliquots are incubated at 37 ${ }^{\circ} \mathrm{C}$ for 5-15 min. To get rid of aggregates of unpolymerized tubulin, the seeds are usually tabletop ultracentrifuged at 20-30 p.s.i for 5 min and resuspended in M2B, diluting them at least 40-fold.

\begin{tabular}{|c|c|c|}
\hline Component & Concentration & Supplier \\
\hline $\begin{array}{c}\text { Biotin-labelled porcine } \\
\text { brain tubulin }\end{array}$ & $0.8 \mathrm{mg} / \mathrm{ml}$ & Cytoskeleton, Inc. \\
\hline $\begin{array}{c}\text { Alexa Fluor 647-labelled } \\
\text { porcine brain tubulin }\end{array}$ & $0.2 \mathrm{mg} / \mathrm{ml}$ & Sigma-Aldrich, D0632 \\
\hline Dithiothreitol (DTT) & $2 \mathrm{mM}$ & Jena Bioscience \\
\hline $\begin{array}{c}\text { Guanosine-5'-[( } \alpha, \beta)- \\
\text { methyleno]triphosphate } \\
\text { (GMPCPP) }\end{array}$ & $1 \mathrm{mM}$ & \\
\hline
\end{tabular}

Table 2.3: Components used to polymerize seeds in M2B buffer

\subsubsection{MAP as an anchor system}

Microtubule-associated proteins (MAPs) are a group of proteins known to stabilize MTs, increase their rigidity and sometimes, even promote MT polymerization [65, 70]. Here, we utilize a structural MAP (containing MAP2 and tau isoforms) that contains MT-binding domains (comprising 3-4 homologous tandem repeat sequences) near the carboxy-terminus of each protein. These binding domains are found to attach the MAPs to the external surface of MTs laterally, via the acidic carboxy-termini of both $\alpha$ - and $\beta$-tubulin. The region opposite the binding domain of MAPs contains several acidic residues that project outward from the microtubules. These projections direct the MT interactions with further components [95, 59].

As a microtubule-organizing centre, we make use of micropillars of diameter 5 $\mu \mathrm{m}$ assembled in a microfluidic device as shown in Figure 2.5 and the steps involved are as follows: 
1. The microfluidic stamp on PDMS (Polydimethylsiloxane) is plasma cleaned and attached to a cleaned glass coverslip according to standard procedures.

2. A $1 \mathrm{mg} / \mathrm{ml}$ MAP solution is flowed through the tubing, avoiding bubbles and incubated at room temperature for 15 min to allow MAP adsorption onto the pillars. The chamber is then washed twice with M2B.

3. Ultracentrifuged microseeds are introduced into the chamber and incubated for $30 \mathrm{~min}$. The washing step is repeated to remove excess seeds that aren't adsorbed on the surface.

4. The standard MT polymerization mix is injected and incubated at $37^{\circ} \mathrm{C}$ for 45 min and washed with M2B that contains taxol.
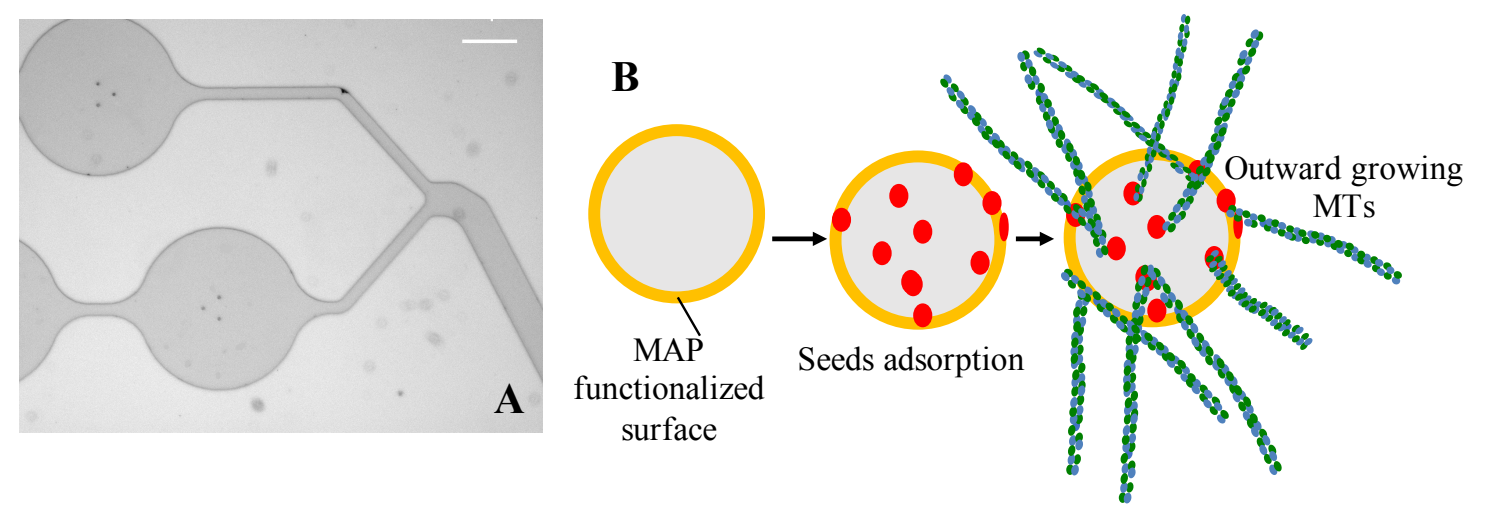

Figure 2.5: (A) A microscopic image of the $5 \mu \mathrm{m}$ micropillars using a $4 \times$ objective. Scale bar : $100 \mu \mathrm{m}$ (B) Schematic of MAP adsorption and growth of MTs from seeds as nucleators.

\subsubsection{The UV Micropatterning technique}

Another method of attaching MTs on a surface involves the technique of UV micropatterning. Micropatterning is a relatively novel technique that allows polymerization of different structures in a reproducible manner for the observation of a global network formation and interaction of single filaments of any kind [7, 79]. The process briefly involves growing MTs on micropatterned features made by printing adhesive micropatterns on a uniformly repellent layer.

This layer is generated by using a doubly passivated surface through the silanePEG and PLL-PEG treatments described in section 2.6. The micropatterns are further coated with nucleation promoting factors that enhance MT assembly, while the repellent areas are devoid of MT nucleation. In our case, we make use of biotinylated seeds that attach to a neutravidin-coated micropattern by the well-known 
biotin-avidin interaction followed by MT polymerization. The experimental procedures to create micropatterns on glass coverslips and grow MTs are described in the following sections.

\section{Design features of Chrome mask}

To design a photomask with precise size indications to create the micropatterns, Autodesk AutoCAD (2016) software was used. Due to irradiation with deep UV light, the photomask needs to be transparent to wavelengths lower than $200 \mathrm{~nm}$. Hence, synthetic quartz was used as the material. While choosing the dimensions of the micropattern motifs, experimental conditions of the nucleation template to be used need to be taken care of. If the nucleation area is too large, several primer nucleation events need to occur to cover the entire surface, impacting the timing and homogeneity of the developing network. This could also trigger faster depletion of proteins and their sinking into non-essential zones. Contrary to this, a small nucleation region may not be reproducibly covered. Additionally, we ensured that the distances between repeated motifs are 50 - $150 \mu \mathrm{m}$ taking into account the lengthscale of potential MT growth. The chrome mask was printed by Compugraphics photomask solutions, Jena and checked for defects.
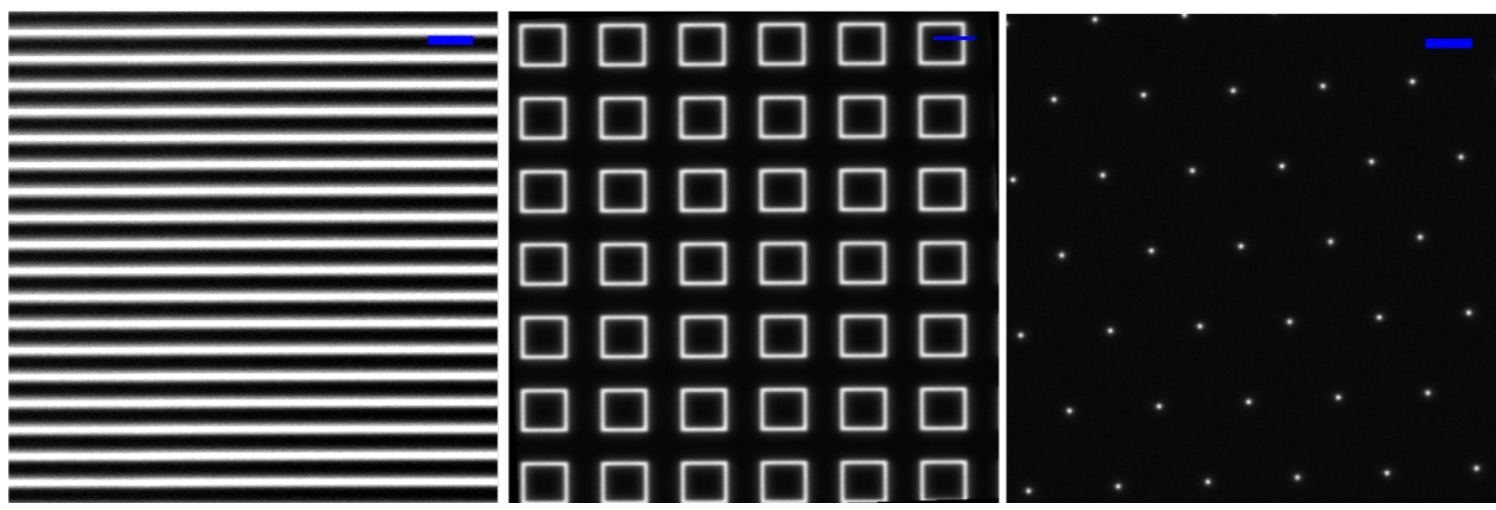

Figure 2.6: Microscopic images of the stripes, squares and dot patterns on the photomask at $10 \times$ magnification. Scale bar: $100 \mu \mathrm{m}$

\section{Micropattern fabrication and functionalization}

We describe the procedure to print micropatterns on glass, functionalize them with neutravidin and grow MTs:

1. Before the experiment, glass coverslips are cleaned and coated with silane-PEG according to Section 2.6.2. The chrome mask is washed consecutively with DI water, isopropanol and dried under filtered airflow. The chrome mask surface 
that will be in contact with the silane-PEG side of the coverslip should be free of any impurity to ensure tight contact.

2. The UV oven cleaner (Jelight, U.S.A) is preheated for 2 min to ensure reproducible irradiation at constant power. The photomask is placed on the holder and a drop of DI water is pipetted over it.

3. The silane-PEG side of the coverslip is placed on the waterdrop to ensure tight contact. This mask-coverslip pair is inverted onto the holder. We use water to ensure tight contact between the surfaces to obtain well-defined micropatterns.

4. The chrome mask is UV-irradiated for 5 min to complete the patterning procedure, illustrated in Figure 2.7.

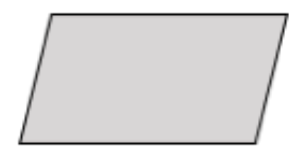

1. Cleaning

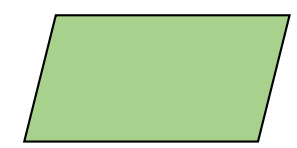

2. PEG-silane coating
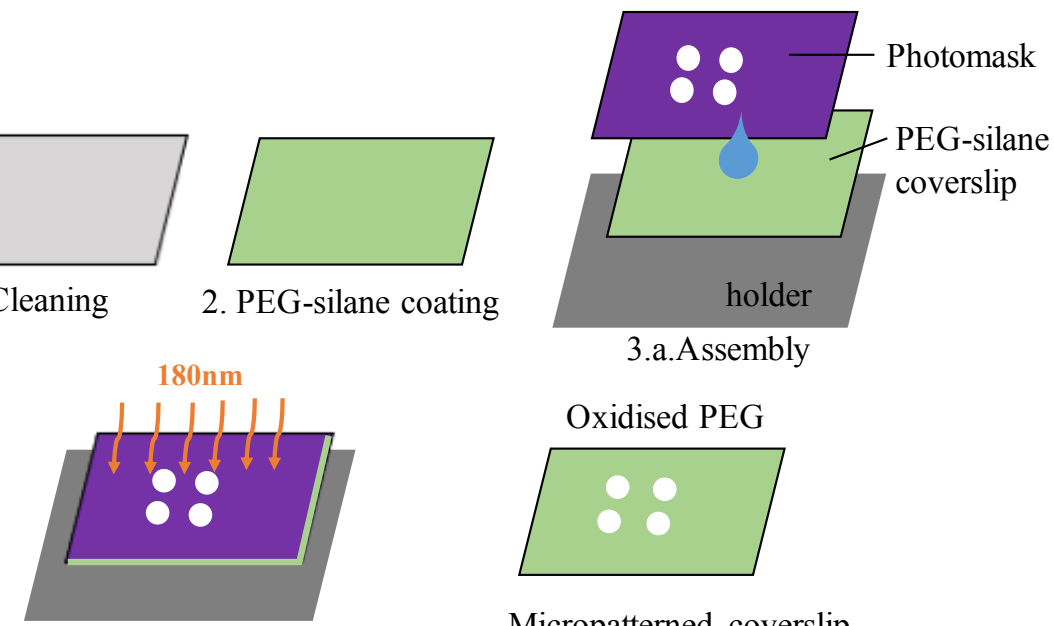

3.b.Deep UV insolation

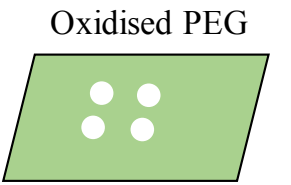

Micropatterned coverslip

Figure 2.7: Overview of the main steps involved in generating micropatterns

Once the micropatterns are fabricated, various steps are involved to initiate MT growth and elongation (Figure 2.8):

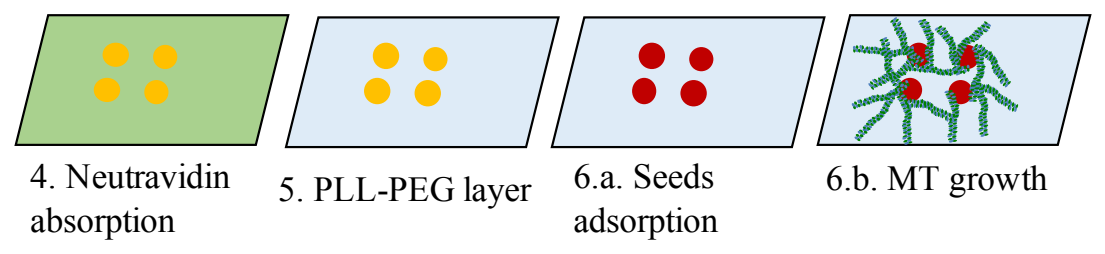

Figure 2.8: Steps toward MT growth and elongation on micropatterns

1. The flow chamber is assembled with a silane-PEG passivated coverslip and the patterned coverslip facing each other using 2 bands of $50 \mu \mathrm{m}$ thick double-sided tape (Nitto Denko, Japan). 
2. $50 \mu \mathrm{g} / \mathrm{ml}$ of neutravidin (ThermoScientific) is pipetted into the chamber and incubated for $15 \mathrm{~min}$. The perfusion chamber is washed with 10 volumes of $\mathrm{M} 2 \mathrm{~B}$.

3. A $0.1 \mathrm{mg} / \mathrm{ml}$ solution of PLL-PEG is pipetted into the chamber and incubated for $10 \mathrm{~min}$. This refers to the double passivation technique that ensures minimal protein depletion on the surface.

4. The flow chamber is now washed with 20 volumes of M2B.

5. GMPCPP-stabilized seeds are added and incubated for at least $15 \mathrm{~min}$.

6. The chamber is washed with 20 volumes of M2B containing $0.5 \%$ BSA.

7. The polymerization mix to induce MT elongation is separately prepared with a tubulin concentration of $1 \mathrm{mg} / \mathrm{ml}$ (Volumes are accordingly adjusted from the standard protocol) and added into the chamber.

8. The chamber is placed in a humid sample box to prevent evaporation from the open sides and incubated at $37{ }^{\circ} \mathrm{C}$ for $30 \mathrm{~min}$. Further, it is washed several times with taxol-containing M2B to stabilize and get rid of excess, unattached microtubules and imaged.

\subsection{Computational methods}

\subsubsection{Filament tracking using Jfilament}

To track filaments in our image processing steps to track filaments in time-series images, we make use of a semi-automatic, open-source tracking software called Jfilament [94], available as an ImageJ plugin. Corresponding to microtubule images, Jfilament uses active contours or 'snakes' (parametric curves) to quantify the filaments by segmentation in two dimensions. When these snakes are placed on an image, they deform actively to make the snake smooth by eliminating abrupt changes in direction. The snakes are then overlapped exactly over the salient image features (Figure 2.9). This software was used for time-lapse image sequences by a continuous adjustment of the associated stretching and smoothing parameters of the tracked snakes.

\subsubsection{Particle tracking algorithms}

To track high densities of tracer particles within active MT networks, we made use of a MATLAB code written and modified for our purpose by Dr Jan Molacek (Max 


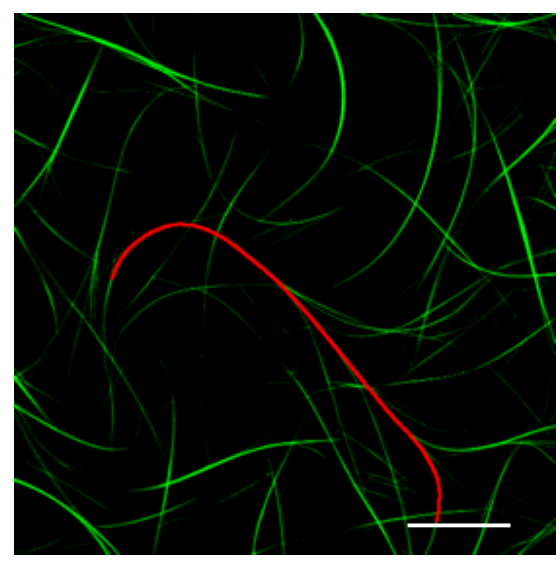

Figure 2.9: Exemplary image of a microtubule bundle network showing the generated track (red) in Jfilament. Scalebar : $50 \mu \mathrm{m}$

Planck Institute for Dynamics and Self-Organization, Göttingen). The algorithm is a simplified version of an in-house tracking code developed by Haitao Xu and others [76], restricted to two dimensions.

For each frame, the locations of tracked objects are determined by first subtracting a $7 \times 7$ window-averaged intensity from each pixel to remove local variations of illumination. This is followed by searching for local extrema of intensity and collecting all neighbouring pixels above a certain intensity threshold into a connected set that corresponds to the image of the object. The exact position of each object is then calculated as the weighted mean position of the pixels within the connected set, weighted by their intensity. The newly extracted positions are then assigned to the existing trajectories by a greedy algorithm. At this step, the trajectories are extended in reverse order of length, by adding the object closest to their extrapolated position if there is one within a given radius. New trajectories are initiated from a triplet of unassigned object locations within the last three frames. The final tracks are obtained as $x$ and $y$ coordinates and used for further calculations.

Some datasets required the generation of velocity maps for flow quantification. We made use of Particle Imaging Velocimetry (PIV) through PIVLab, an opensource GUI based software [100]. The velocimetry technique involves three stages: image pre-processing, vector generation and post-processing. For our purposes, we made use of CLAHE (Contrast limited adaptive histogram equalization) as a preprocessing step, followed by vector validation and interpolation of missing values. As a final step, smoothing was performed through median filtering and velocity maps were plotted. 


\subsubsection{Orientation measurements using OrientationJ}

The ImageJ plugin OrientationJ was used to extract orientation angles of MTs and generate maps of different regions of an image. It is based on the evaluation of the gradient structure tensor in a local neighbourhood of a region of interest (ROI). It first extracts the weighted inner product of a window centred on a local ROI. The derivative of this product was used to find the direction along which its value is maximised over the ROI. This resulted in a histogram of orientation distributions with significant peaks in the cases of directed MT images. Coherency values were varied between $0-0.5$ to focus on extracting local image features and eliminate artefacts due to background noise.

\subsubsection{Estimation of flexural rigidity}

Calculations related to microtubule bending dynamics begin with its flexural rigidity $(E I)$ as its starting point. The flexural rigidity or bending stiffness can be defined as a quantification of a filament's resistance to bending forces 63 .

According to the worm-like chain model, we can assume MT filaments to be continuous strings resistant to any kind of bending deformation.

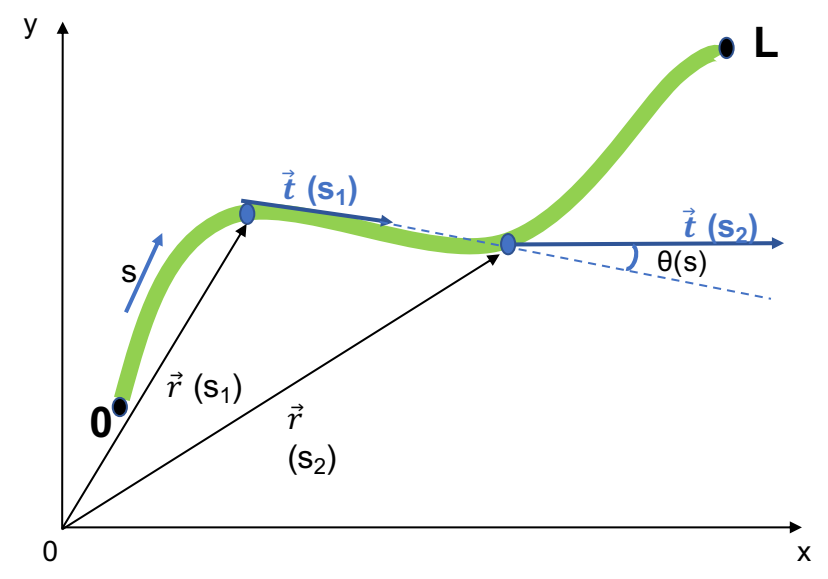

Figure 2.10: A schematic of the worm-like chain model

Figure 2.10 shows the model of a thin semi-flexible rod of length $L$. We represent its shape completely by the tangent angle $\theta(s)$ at every ' $s$ ' along $L(0 \leq s \leq L)$. Let us denote the curvature at every continuous point along $L$ by $\frac{d \theta}{d s}$. If the radius of curvature is much greater than the filament diameter, the bending energy per unit length is given by

$$
\frac{d U}{d s}=\frac{1}{2} E I\left(\frac{d \theta}{d s}-\frac{d \theta^{0}}{d s}\right)^{2}
$$

where $\theta^{0}(s)$ is the shape of a relaxed rod due to intrinsic bending and in the absence of thermal forces. This expression takes us back to the energy of bending in a 
beam. The product EI is the above-defined "flexural rigidity", composed of Young's Modulus (E) and the second moment of cross-sectional area (I), given by

$$
I=\int_{A} y^{2} d A
$$

Integrating over Eq. 2.3, we get the total energy of bending for an infinitely thin rod in its plane

$$
U=\frac{1}{2} E I \int_{0}^{L} d s\left(\frac{d \theta}{d s}-\frac{d \theta^{0}}{d s}\right)^{2}
$$

To find an estimate for the EI of the polymerized microtubules, it becomes essential to find the persistence length, the arc length above which the filament bends, $l_{p}$.

Consider a short beam of arc length $s$, its bending energy is

$$
U_{\text {bend }}=\frac{E I}{2 s} \theta^{2}
$$

We begin with tangent-tangent correlation function along a filament

$$
g(s)=<t(\vec{s}) \cdot t(\overrightarrow{0})>
$$

with

$$
t(\vec{s})=\frac{\partial \vec{r}(s)}{\partial s}
$$

Taking the tangent of one end of the beam along $z$-direction, the tangent-tangent correlation function becomes

$$
g(s)=<\cos \theta(s)>
$$

If the deflection angles $\theta$ are assumed to be very small i.e., $\theta \leq 1$, the cosine function can be expressed as a Taylor series. Ignoring higher order terms,

$$
g(s)=\left\langle 1-\frac{\theta^{2}(s)}{2}\right\rangle
$$

If we compute the ensemble average by summing over all possible orientations of the tangent vector at $s$, (which in three dimensions, traces out a unit sphere), using Eq 2.6 and the spherical coordinate system $(\mathrm{r}, \theta, \phi)$,

$$
<\theta^{2}(s)>=\frac{1}{Z} \int_{0}^{2 \pi} d \phi \int_{0}^{\pi} d \theta \sin \theta \theta^{2} e^{-\frac{E I}{2 k_{B} T s} \theta^{2}}
$$

where

$$
Z=\int_{0}^{2 \pi} d \phi \int_{0}^{\pi} d \theta \sin \theta e^{-\frac{E I}{2 k_{B} T s} \theta^{2}}
$$

is the normalization factor.

Eq. 2.11 and 2.12 gives us

$$
<\theta^{2}(s)>=\frac{1}{Z}\left(-\frac{2 \pi k_{B} T s}{I} \cdot \frac{\partial Z}{\partial E}\right)=-\frac{2 \pi k_{B} T s}{I} \cdot \frac{\partial \ln Z}{\partial E}
$$


For small angles, $\sin \theta \approx \theta$. Integrating $\mathrm{Eq} 2.12$,

$$
Z=\frac{2 \pi k_{B} T s}{E I} \int_{0}^{\infty} e^{-u} d u
$$

by making a change of variables $u=\frac{E I}{2 k_{B} T s} \theta^{2}$ and becomes

$$
Z=\frac{2 \pi k_{B} T s}{E I}
$$

From Eq. 2.13, we derive the mean-squared average of $\theta(s)$

$$
<\theta^{2}(s)>=\frac{2 k_{B} T s}{E I}
$$

Substituting Eq2.16 in Eq. 2.10,

$$
g(s)=1-\frac{k_{B} T}{E I} \cdot s
$$

Our tangent is of unit length, $g(0)=1$. The two tangent vectors will be independent for $s$ much larger than $l_{p}$ and $g(s) \rightarrow 0$. Therefore, the tangent-tangent correlation function is an exponential function of the form

$$
g(s)=e^{-\frac{k_{B} T}{E I} s}
$$

Since this factor characterises how quickly the shape changes along a filament, we equate it as a definition of $l_{p}$.

$$
g(s)=e^{-\frac{s}{l_{p}}}
$$

which implies

$$
l_{p}=\frac{E I}{k_{B} T}
$$

This expression for determining the persistence length of a MT (or any filament, in general) applies to MTs that undergo thermal fluctuations.

\section{Filament shape decomposition into Fourier modes}

Following previous studies to calculate EI [35], we make use of a Fourier decomposition method for thermally bending MTs polymerized according to conditions described in Section 2.1. By resolving the thermally fluctuating shape of the filament, the amplitude and its corresponding fluctuations (variance) provided an estimate for EI as described below. The shape of an MT, characterised by $\theta(s)$ can be expressed as a superposition of a large number of Fourier modes i.e.,

$$
\theta(s)=\sum_{n=0}^{\infty} \theta_{n}(s)=\sqrt{\frac{2}{L}} \sum_{n=0}^{\infty} a_{n} \cos \left(\frac{n \pi s}{L}\right)
$$


Differentiating 2.21 and plugging into 2.5 ,

$$
U=\frac{1}{2} E I \sum_{n=1}^{\infty} \frac{2}{L}\left(\frac{n \pi}{L}\right)^{2}\left(a_{n}-a_{n}^{0}\right)^{2} \int_{0}^{L} \sin ^{2} \frac{n \pi s}{L} d s
$$

We can now write the bending energy as a quadratic summation of these coefficients

$$
U=\frac{1}{2} E I \sum_{n=1}^{\infty}\left(\frac{n \pi}{L}\right)^{2}\left(a_{n}-a_{n}^{0}\right)^{2}
$$

The zero-order mode $a_{n}^{0}$ denotes the average orientation of the MTs and doesn't contribute to the bending energy. The equipartition theorem states that each quadratic term in Eq. 2.23 contributes to an average $\frac{k_{B} T}{2}$. Hence,

$$
\operatorname{var}\left(a_{n}\right)=<\left(a_{n}-a_{n}^{0}\right)^{2}>=\frac{k_{B} T}{E I}\left(\frac{L}{n \pi}\right)^{2}
$$

The variation/fluctuation in amplitude of each mode $(n \geq 1)$ provides an independent estimate of flexural rigidity, EI. From Eq. 2.24, one can relate the persistence length, $L_{p}$ to variance as

$$
L_{p}=\frac{L^{2}}{n^{2} \pi^{2} \operatorname{var}\left(a_{n}\right)}
$$
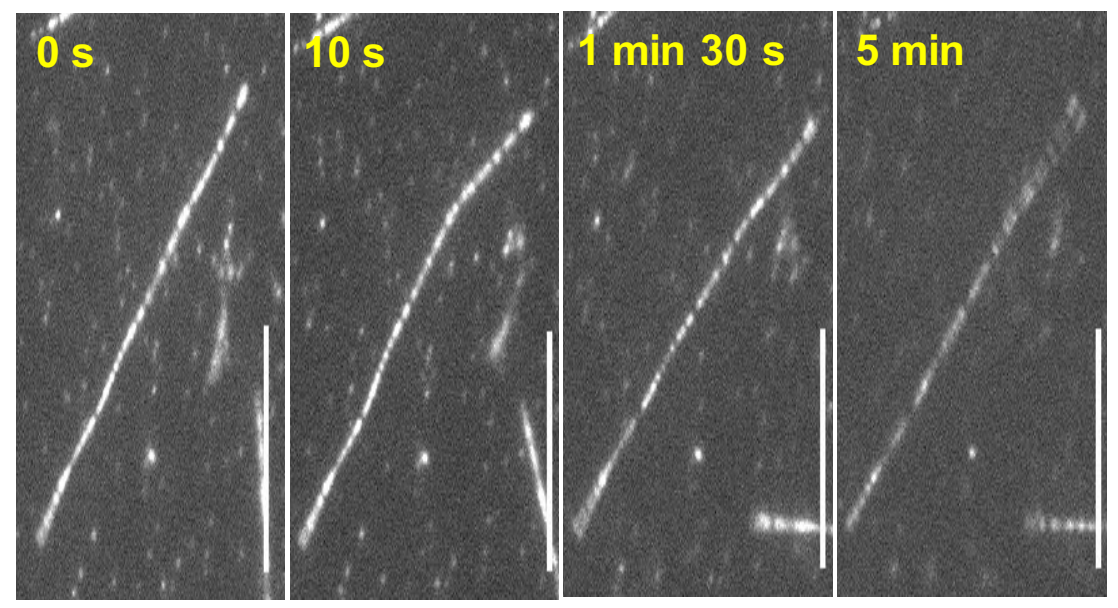

Figure 2.11: Thermally fluctuating microtubules imaged for a period of $5 \mathrm{~min}$. Scale bar : $10 \mu \mathrm{m}$

To perform the Fourier decomposition technique, we implemented code in MATLAB by first loading the snake coordinates of the filaments extracted from Jfilament and dividing each snake into many segments. Further, the tangent angle that each of these segments made with the $\mathrm{x}$-axis and the amplitudes of the modes were calculated (Eq. 2.21).

1. In the implemented algorithm, for $\mathrm{N}+1$ points along with a tracked snake in two dimensions, considering $\mathrm{N}$ segments that connect these points, the distance between these bonds was given by 


$$
\Delta s_{i}=\left[\left(x_{i+1}-x_{i}\right)^{2}+\left(y_{i+1}-y_{i}\right)^{2}\right]^{1 / 2}
$$

2. The tangent angles were given by

$$
\theta_{i}=\tan ^{-1}\left(\frac{y_{i+1}-y_{i}}{x_{i+1}-x_{i}}\right)
$$

3. The vector containing the tangent angles are used in the Fourier analysis. The coefficients $\mathrm{a}_{n}$ are calculated using:

$$
a_{n}=\sqrt{\frac{2}{L}} \int_{s=0}^{L} d s \theta(s) \cos \left(\frac{2 \pi s}{L}\right)
$$

This takes the form :

$$
a_{n} \cong \sqrt{\frac{2}{L}} \sum_{i=1}^{N} \Delta s_{i} \theta_{i} \cos \left(\frac{n \pi}{L} s_{i}^{m i d}\right) \quad, n=1, \ldots ., N-1
$$

where

$$
L=\sum_{i=1}^{N} \Delta s_{i}
$$

and

$$
s_{i}^{m i d}=\Delta s_{1}+\Delta s_{2}+\ldots+\Delta s_{i-1}+\frac{1}{2} \Delta s_{i}
$$

4. By determining the amplitude, its variance was plotted against the Fourier mode number.

In our case, the value of the variance exponentially decays at a mode number $=$ 3 , implying that we could resolve the filament into 3 Fourier modes. $\operatorname{var}\left(a_{n}\right)$ was found to be $0.0334 \mu \mathrm{m}$. Substituting this value into Eq.2.25, we found that $L_{p}$ was $1.2 \mathrm{~mm}$. This range of persistence length agrees with known considerations of MTs as stiff polymers 35 .

To find EI, we make use of the derived value of $L_{p}$ in Eq. 2.20 to get a value of $0.4 \times 10^{-23} \mathrm{Nm}^{2}$ for our case of taxol-stabilised MTs polymerized for $30 \mathrm{~min}$. This is the value we shall employ for all further calculations used in this work. 


\section{Chapter 3}

\section{Results}

This work is centred around the self-assembly of synthetic MT-motor protein systems as a model to study the dynamics of cellular processes such as those described in the introduction. By incorporating these building blocks in our experiments, we were able to visualize and analyze these systems at three different hierarchical levels.

The first level is a direct inspiration from the nature of the actual cellular organization. Cellular interiors are extremely crowded. Their heterogeneous environments are comprised of 'linked' polymer networks that act inter-dependently to aid important cellular dynamics. This generated our interest in exploring the motility of motor clusters and their interactions with MTs, which is the focus of Section 3.1. Further, we studied the large-scale behaviour of such networks when geometrically confined into chambers of different heights, described in Section 3.3.

The second level is to understand the intrinsic working of our model in its standard biochemical environment i.e. by exploiting the "dilute-solution" limit. In Section 3.2, we deal with the behaviour of active filament bundles that are not bound to a surface and free to move in all planes, much like the cytoskeletal filaments in a 3D cellular environment. We go a step further in dilution to reach the single-filament limit and observe its undulatory behaviour, described in Section 3.4.

Thus, our work offers a complete characterization of self-assembling properties and MT-motor protein interactions at various levels of complexity in a hierarchical organization. 
Parts of this chapter and figures have been published in the following journal article (licensed under CC-BY):

"Flagella-like Beating of a Single Microtubule" Andrej Vilfan*, Smrithika Subramani*, Eberhard Bodenschatz, Ramin Golestanian and Isabella Guido, Nano Letters 2019 19(5), 3359-3363

\subsection{Kinesin-embedded microtubule networks}

Our experimental setup results in the generation of dense bundled active networks, the bundles forming due to the depletion effect of PEG (Refer Section 2.2). These bundles contain multiple merged MTs that continuously exhibit a variety of dynamics. It is interesting to understand the origins of this behaviour in terms of motor cluster dynamics.

To this end, we labelled the streptavidin units of the motor protein clusters in the networks to easily track their movement using an epifluorescence microscope (Refer section 2.3.1). The kinesin clusters are randomly distributed within every bundle and are not fixed at a defined position (Figure 3.1). Due to the inclusion of PEG, the resulting MT-MT distance decreases. This proximity allows the kinesin clusters to behave as dynamic cross-linkers. Extensive studies on single filament motion have been characterized by a continuous stepping action of motors, with pauses in between as expected for a stochastic stepper such as kinesin-1 [9]. The tracking of the clusters gave us the possibility to observe and analyze a distinct motor cluster behaviour that hasn't been observed in single filament studies so far.

Interestingly, the tracks exhibit a continuous movement on a bundle with intermediate pauses [46] and characteristic 'jumps' to the neighbouring bundles.

The tracking and characterization of the walking action of kinesin were done as long as it was visible under the microscope, typically for a period of $15 \mathrm{~min}$, including its brief periods of pause. The complete distance travelled by the clusters during this period called the travel length was found to be $93 \pm 22 \mu \mathrm{m}$ (Figure $3.2(\mathrm{~A})$ ). This relatively long distance is due to the architectural arrangement of the MTs in the network. As the distance between MTs in a bundle is dictated by the osmotic pressure due to PEG, there is an increased probability for the kinesins to walk, dissociate from a bundle, but immediately associate onto the next bundle in its vicinity. It is noteworthy to mention that our experimental setup did not enable us to differentiate the motor protein relative to the intrinsic MT bundle movements in bulk. Thus, the distances calculated in these experiments are a result of the MT bundle-motor protein cluster combination. 


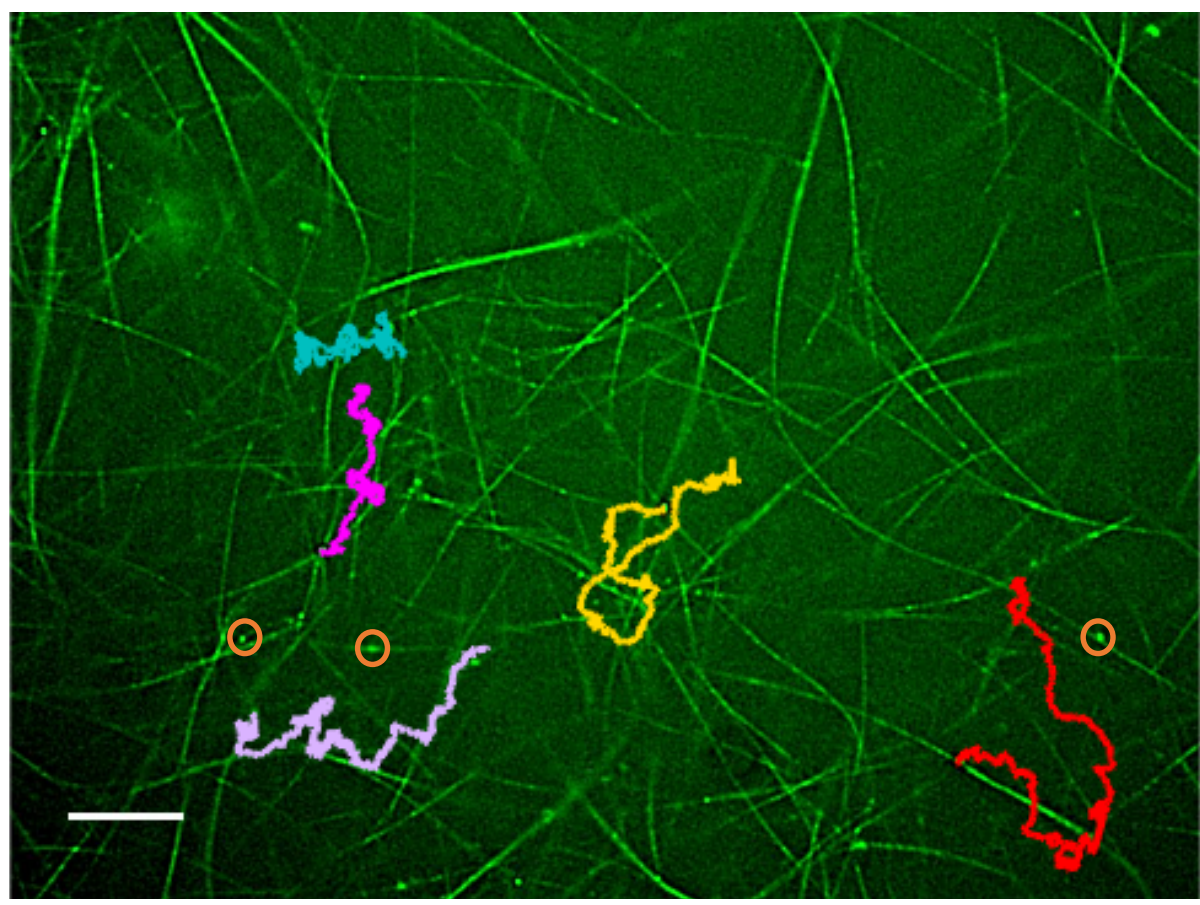

Figure 3.1: Exemplary tracks showing the path travelled by the MT - motor protein clusters across the network for a period of $15 \mathrm{~min}$. Some of the untracked labelled motor proteins can be seen as small green dots encircled in orange. For visual clarity, the Cy-3 streptavidin-kinesin clusters are depicted in green. While most clusters display complex inter-bundle pathways, very few (tracks in turquoise and pink) remain bound to a few MT bundles in the same region for the entire period. Scale bar : $10 \mu \mathrm{m}$

The average velocity of the clusters was calculated to be $0.26 \mu \mathrm{ms}^{-1}$ (Figure $3.2(\mathrm{~B}))$. The velocity of the clusters is considered lower when compared to singlekinesin experiments that displayed velocities of $0.84 \mu \mathrm{ms}^{-1}$ [55, 17]. Our value is however in agreement with previous studies of single kinesin motility on a PEG depleted bundle [16], due to the very reason of motor speeds being affected by the presence of PEG and bundling. According to collective transport studies of multiple kinesins performed by Furuta et al [28] through optical trapping assays, the velocity of a number of motors in proximity is not additive, it remains unaffected by the close spacing between the motors, thus explaining the similar range of $0.2-0.3 \mu \mathrm{ms}^{-1}$ for both single kinesins as well as clusters. It is worth noting that some cluster velocities reach higher values (Figure 3.2(B)) likely due to the apparent momentary jumps that occur during transit to neighbouring bundles as observed under the microscope.

To better explain the cluster movements, we employ a terminology for the two types of motility parameters (as illustrated in Figure 3.3), namely :

(i) Activity, which describes the movement of a cluster as long as it is active through its stepping and jumping action, either while being bound to an individual MT 

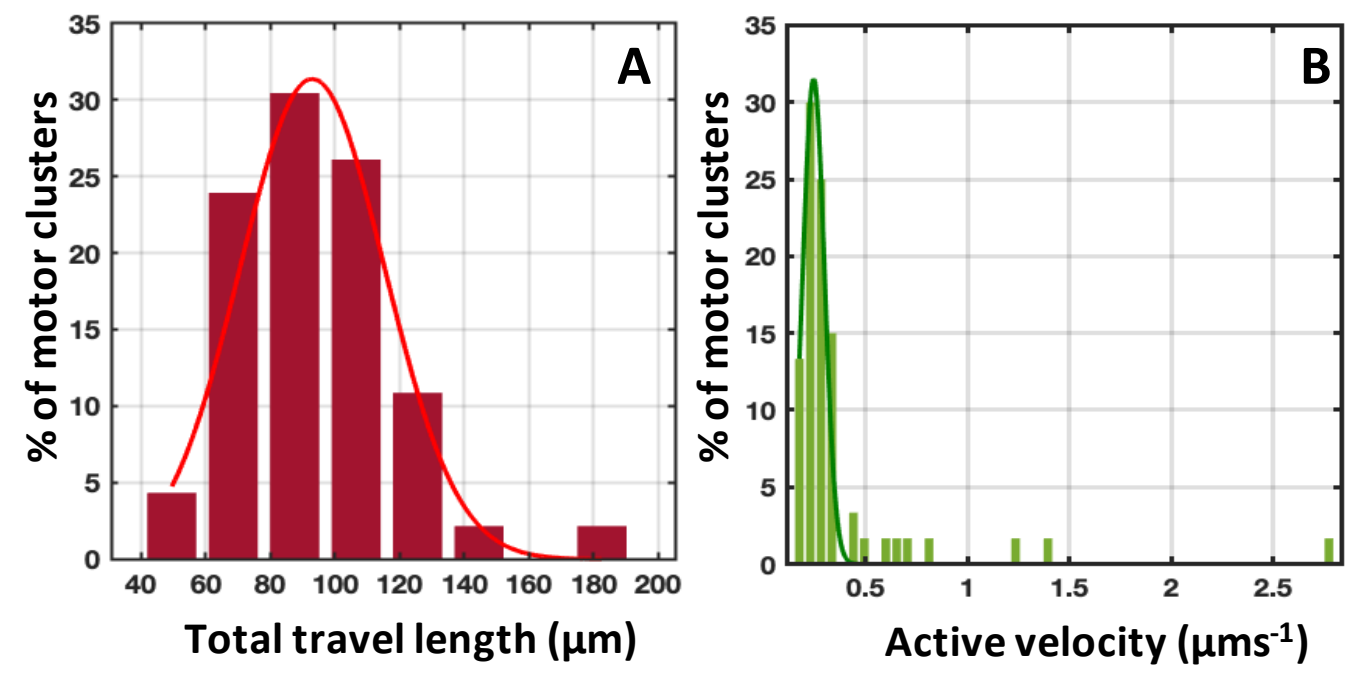

Figure 3.2: Histograms depicting (A)the total distances covered by the kinesin clusters during $15 \mathrm{~min}$ of imaging time and (B) the average velocities of the clusters during their period of activity.

bundle or during its jump and subsequent binding to a proximal bundle i.e. all the active interactions on multiple MT bundles. The periods of pause that may occur during each binding or before the jumps are neglected.

(ii) Association, which describes the movement of a cluster as long as it is bound to an individual MT bundle. This includes the periods of pause or inactivity during its binding on that particular MT bundle.

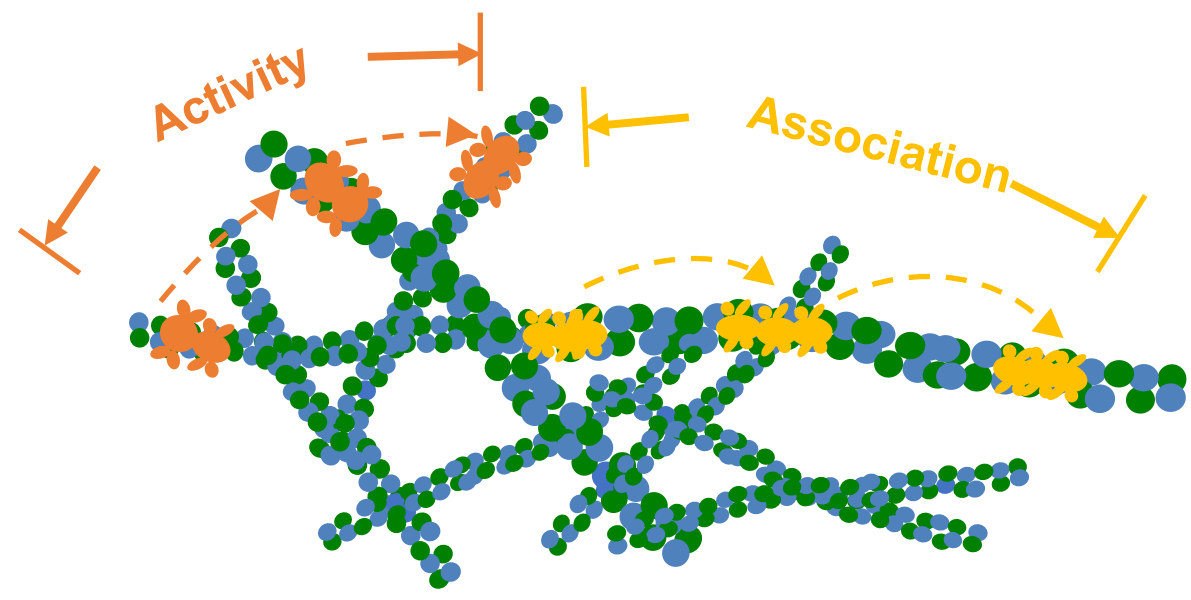

Figure 3.3: A schematic illustration of the 2 modes of motor cluster motility (in yellow and orange), (i) activity and (ii) association on MT networks.

Considering the activity of the clusters, we now define an activity time as the time for an event of walking over multiple bundles i.e. every single event is the time 
period between two pauses of the cluster. The value of this activity time was found to be $17 \pm 0.4 \mathrm{~s}$ (Figure 3.4(A)). The corresponding distance covered by the motor cluster during this time that we call activity length, was found to be $4.03 \pm 0.11 \mu \mathrm{m}$ (Figure $3.4(\mathrm{~B})$ ).
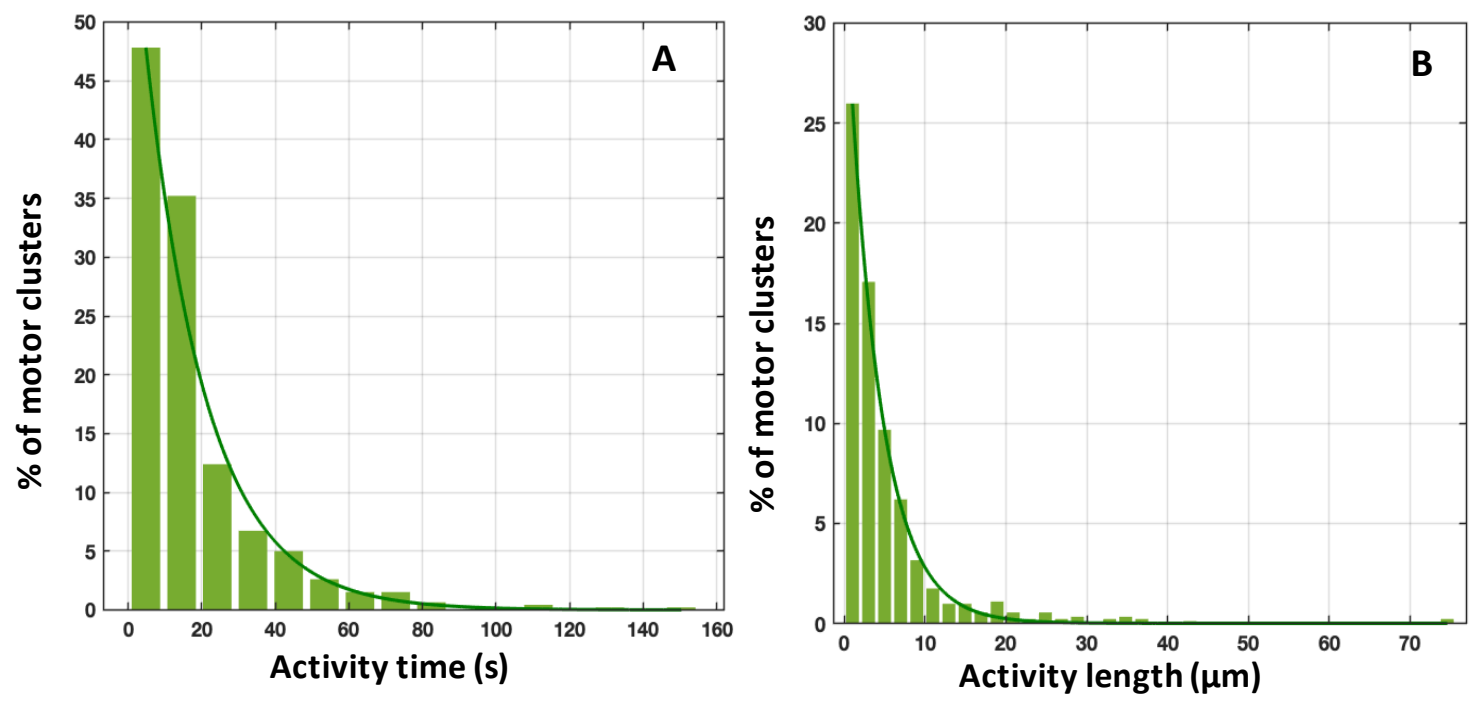

Figure 3.4: Normalized probability distributions with an exponential distribution depicting the (A) periods of activity and (B) activity length i.e. the distance travelled during a period of activity.

Now, considering the cluster motility over an individual MT bundle as long as it is associated with that bundle even during its period of pause, we define its total residence time on a bundle as the association time. The association time was calculated to be $21 \pm 0.2 \mathrm{~s}$ (Figure 3.5(A)). This value is orders of magnitudes greater than the association time of a single kinesin-1, a few $100 \mathrm{~ms}$ [55]. Meanwhile, the corresponding distance travelled by the cluster on that bundle during a period of association, the association length was found to be $4.1 \pm 0.1 \mu \mathrm{m}$ (Figure 3.5(B)). It is known that on average, kinesin takes 150 steps which could cover a distance between 0.4-1.2 $\mu \mathrm{m}$ (directly dependent on motor concentration) called the run length, before dissociating or detaching from a MT [41, 9, 46, 102, 99]. Due to multiple motors in the cluster, we observe an enhanced association length $(4.1 \pm 0.1 \mu \mathrm{m})$ because of the higher probability of the cluster configuration to elongate its residence on the bundle [84, 60]. Another interesting observation is the dwell time of the clusters i.e. the total pause time of the cluster while associated on a bundle. Standard kinesin-1 experiments have revealed a dwell time of 0.5-2 s [99]. In the case of motor cluster experiments, we observe that every cluster pauses at least once time during its entire path. The number of times a cluster pauses in a network is much greater than on a single filament.

The kinesin-1 complex used for our studies is tetrameric, meaning that every 

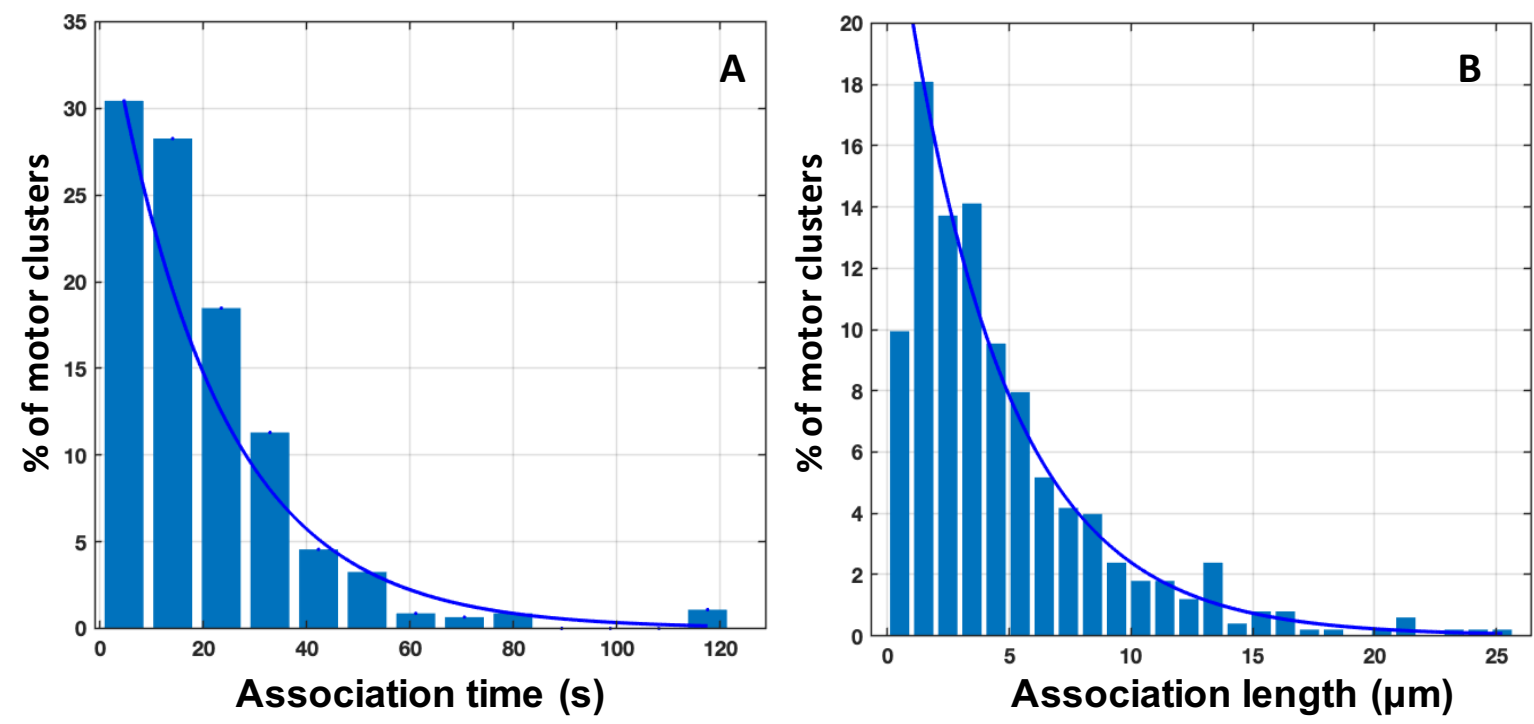

Figure 3.5: Normalized probability distributions with an exponential distribution depicting the (A) periods of association and (B) association length i.e. the distance travelled during a cluster's residence on one bundle.

cluster can contain up to four single motors. This may suggest that the simultaneous pausing of some motors within a cluster could reduce the probability of unbinding of other motors within a cluster [90]. It implies that every motor may be sensitive to pauses of other motors that make up the cluster, hence increasing its pause time. In the experimental observations, even though a cluster may pause and dissociate from the end of a bundle, the availability of the next track (a neighbouring sliding MT) increased its probability to walk and hence, pause several times.

An overview of the association and activity parameters provides us with a relation between the walk, pause and jump activities of the kinesin clusters when associated with multiple active bundles. While the activity and association times are comparable in the range of 17 and 21 s respectively, the similar values of the activity and association lengths offers an interesting insight. It would imply that despite travelling complicated paths through continuous association and dissociation between multiple microtubule bundles, the motor cluster on average travels a similar distance during its activity (on multiple bundles) as well as an association (on an individual bundle) during its motility. 
An understanding of the basics of motor dynamics in networks of randomly distributed MT bundles now paves the way to understand their effect on individual MT bundles. In the experiments described here, we should note that the activity and association parameters pertaining to the kinesin clusters do not distinguish the movement of the MT bundles. The values obtained here are a characterization of the combined dynamics of bulk motor-MT bundles. Further analysis that distinguishes the dynamics of kinesin clusters could provide us a better understanding of the observed differences in the activity and association parameters. 


\subsection{Bending dynamics of individual bundles}

In this section, we deal with a complementary approach to understanding better the mechanical behaviour of MTs due to motor clusters. Building upon previous studies that make use of depleted MT-motor networks [89, 88, 38, we take a step further to dilute such networks to a single bundle level that can be tracked to understand its individual bending behaviour.

\subsubsection{Experimental results}

We generated MT-motor protein networks that were further diluted to view single or very few active MT bundles (Refer Section 2.3.2). Importantly, these active bundles have no fixed ends and are free to move in all planes. The experimental observations display active bundles that continuously bend, buckle, merge, disassemble and reassemble with time as seen in Figure 3.6 .

It is important to note that the individual bundles have resultant lengths of 100-300 $\mu \mathrm{m}$. However, they are composed of bunches of merged single MTs in the length range of $2-55 \mu \mathrm{m}$.

As explained in section 2.8.2, we quantify a single filament's (MT) resistance to bending in terms of a constant, called its flexural rigidity (EI), whose value for our case was found to be $0.4 \times 10^{-23} \mathrm{Nm}^{2}$. The characterization of multiple assembled MTs as a bundle is done using the worm-like bundle (WLB) model proposed by Heussinger et al [43, 8]. Accordingly, bundles can be defined as an assembly of multiple semiflexible filaments, whose mechanical properties can be described by those of its constituent filaments at a microscopic level. A bundle's bending stiffness (a measure of its flexibility) is generated due to the competition between the bending or stiffness of its filaments and their resulting sliding motion. Additionally, the flexural rigidity of microtubule bundles (with PEG and motors in the absence of ATP) was found to be in a similar range as that of single filaments, with a value of $0.7 \times 10^{-23} \mathrm{Nm}^{2}$. By this assumption, we can approximate the bundles to be single, continuous elastic biopolymers. The bundles are dilute and few in number with minimal background noise, so we make use of Jfilament 94] as a tool to track and smooth individual snakes to generate their coordinates.

To quantify the bending dynamics of the MT bundles, we divide a continuous track of the bundle into segments of smaller arc lengths $(s)$ and calculate the tangent angles $(\theta)$ between these segments. Similar to a filament's resistance to bending under thermal forces that is quantified through its persistence length, here we define a dynamic persistence length for a bundle continuously bends and buckles over time. We can find a characteristic length along the filament at which the tangent-tangent 


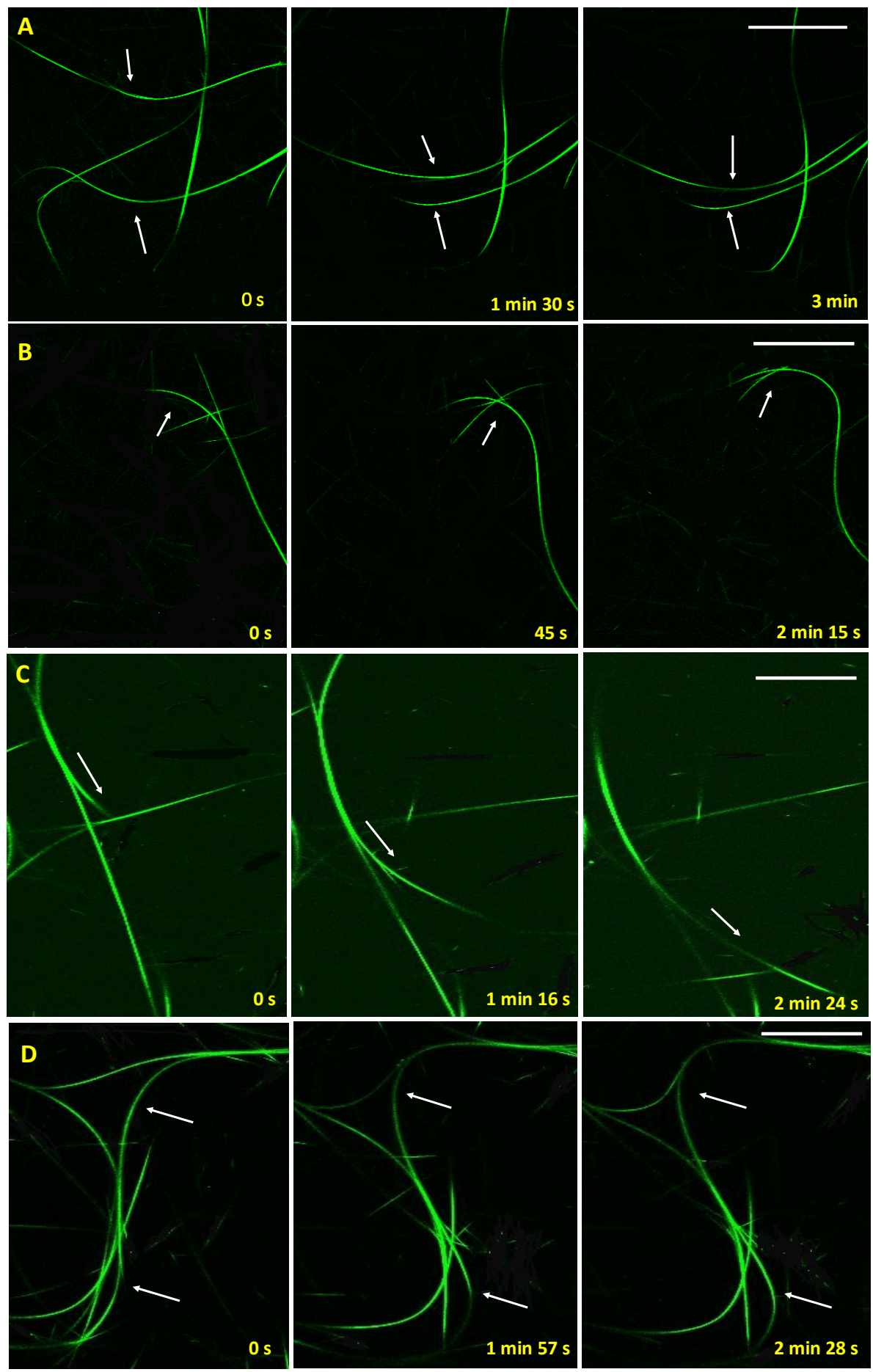

Figure 3.6: Time-lapse images showing motor cluster-MT bundles that demonstrate (A) merging (B) bending (C) inter-bundle sliding and (D) buckling dynamics. Scale bar : $50 \mu \mathrm{m}$.

correlation collapses. As the arc length $s$ is increased, the time average of $\cos [\Delta \theta]$ decreases exponentially. We define this characteristic dynamic persistence length as the active bending length $(\mathfrak{i})$, which provides an estimate of an individual bundle's 
flexibility due to the motor-induced movement:

$$
<\cos [\Delta \theta]>=\exp (-s / 2 \mathfrak{i})
$$

From the exponential decay (Figure 3.7) of the time-averaged angular change of $\cos [\Delta \theta]$, the inverse of the slope gives us $\mathfrak{b}=84.2 \pm 5.9 \mu \mathrm{m}$. This small range of $\mathfrak{i}$ reveals that although the bundle length is heterogeneous as seen in Figure 3.6, with length distributions in the range 100-300 $\mu \mathrm{m}$, their bending occurs over a smaller length range of $80-90 \mu \mathrm{m}$.

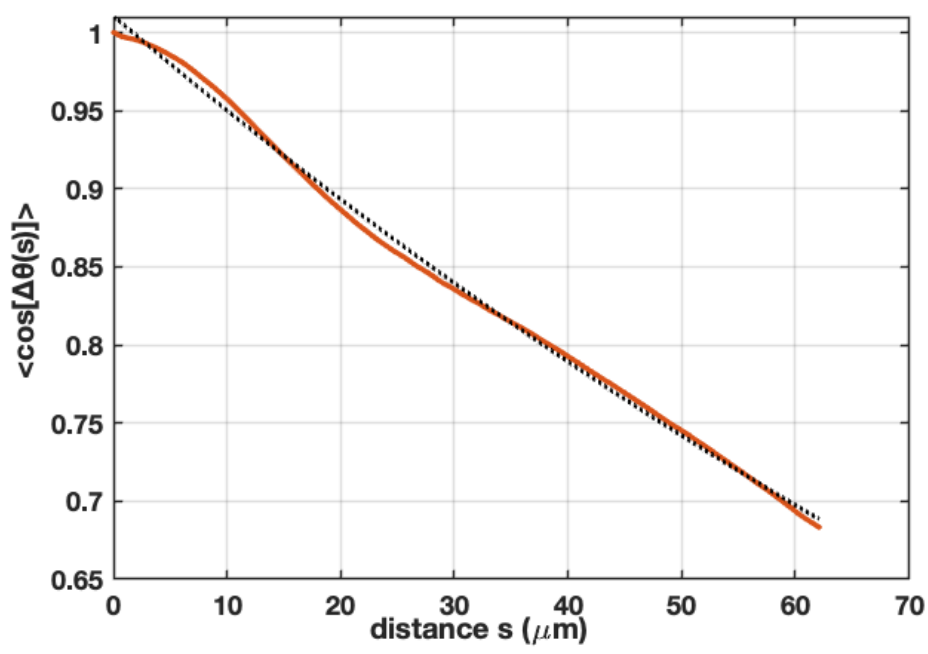

Figure 3.7: An exponential fit (in black) to the mean tangent-tangent correlation graph (in red) of the moving bundles depicts a time-dependent active bending length in the range $80-90 \mu \mathrm{m}$.

Quantifying the bending activities of the individual MT bundles results in questions regarding the forces generated due to the movement of the randomly-distributed motor clusters. To answer this, we estimate the force density of the MT bundles. Once again, considering the bundles as single continuous filaments from the wormlike bundle (WLB) model, we estimate the force density to be :

$$
f=\frac{\left(\frac{d K}{d s}\right) n E I}{d}
$$

where $d K / d s$ refers to the rate of change of curvature $\mathrm{K}$ along the length of the MT bundle, $n$ is the number of MTs that constitute a bundle and $d$ is the average size of the kinesin-1 cluster that moves between and separates the MTs within a depleted bundle. The value of $n$ is assumed to be 4, according to the molecular weight of PEG that we use $(20 \mathrm{kDa})$ and confirmed from structural x-ray scattering experiments [75, 44]. The average size of a kinesin-1 cluster is $\sim 75 \mathrm{~nm}$ and EI $=$ $0.4 \times 10^{-23} \mathrm{~N} \mathrm{~m}^{2}$. 
As an example, Figure 3.8 (A) depicts a bundle whose end's curvature increases due to motor behavior. The curvature is calculated for the duration of its movement and is depicted in a kymograph in Figure $3.8(\mathrm{~B})$, where the tip's curvature is higher than the rest of the bundle (yellow region). We estimated the force densities for all

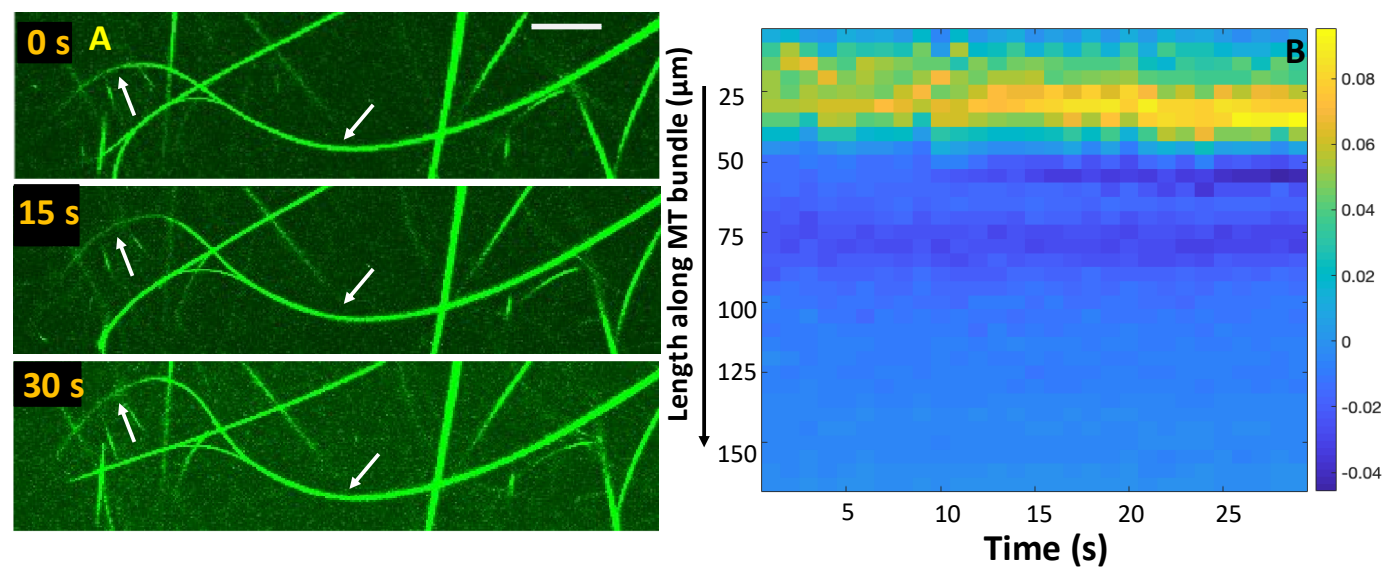

Figure 3.8: (A) Time-elapse of an MT bundle that curves at the tip (Scale bar : 10 $\mu \mathrm{m})$ and (B) its corresponding kymograph depicts higher curvature in this region as a proof of forces acting along its length.

the experimental cases by making use of Eq. 3.2 and depict it in a histogram. While force densities can reach a few hundreds of $\mathrm{pN}_{\mu} \mathrm{m}^{-1}$, the average value was found to be $63 \mathrm{pN} \mu \mathrm{m}^{-1}$ (Figure 3.9.

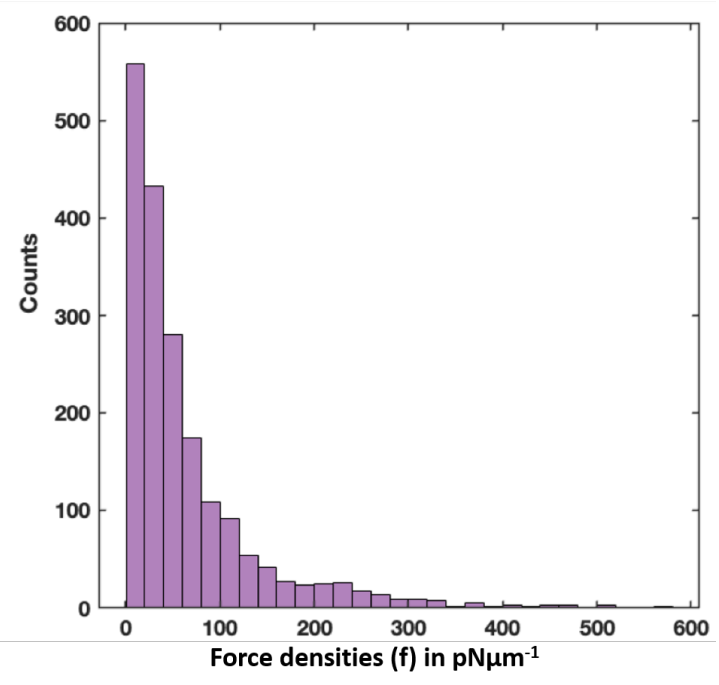

Figure 3.9: Histogram of force densities from seven sets of experimental observations.

An interesting behaviour of the MT-motor systems was its ability to bend and buckle in 3D. As a preliminary investigation of this 3D behaviour, we employed a multi-plane imaging technique to acquire volumetric images of the bundling activity. The imaging was performed in the group of Prof. Dr Jörg Enderlein (III. 
Physical Institute, Georg-August-Universität Göttingen). The multi-plane imaging setup consists of a wide-field microscope along with a multi-plane beam splitter that can apply eight distinct optical paths. Through this method, we could simultaneously acquire 100 volume images per second of up to eight different planes. The inter-planar distance was $650 \mathrm{~nm}$ and the total range in depth was $\sim 5 \mu \mathrm{m}$. The experimental observations depicted the bending and transitions of the bundles into multiple planes.
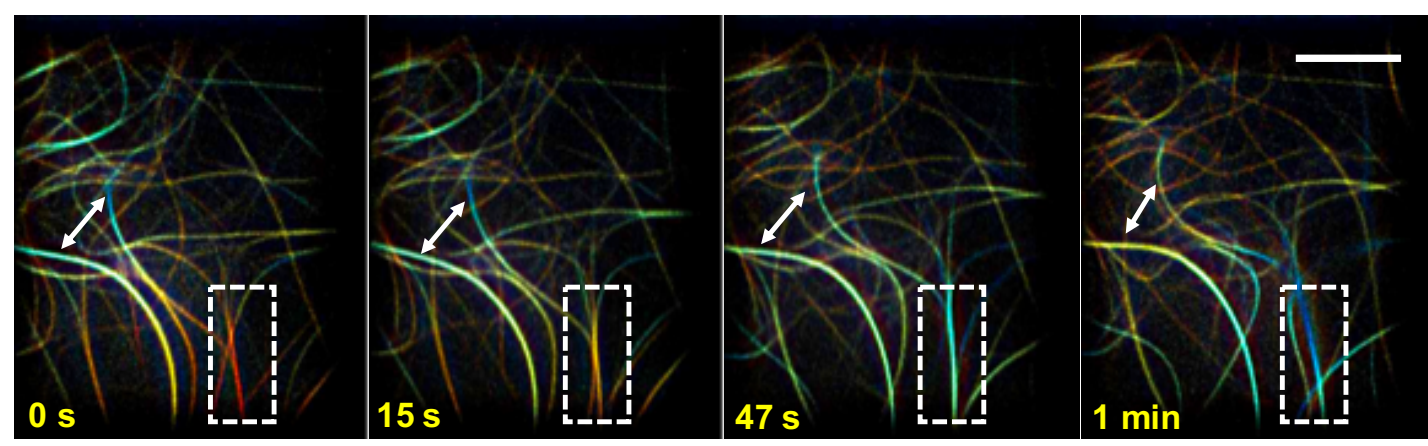

$5 \mu \mathrm{m}$

Figure 3.10: 3D imaging reveals bundle dynamics. Scale bar : $10 \mu \mathrm{m}$

As the next step in this direction, a 3D tracking analysis of the microtubule bundles could provide us more information on the true curvature of the filaments and provide a more accurate description of the bending activity that we reported in 2D. In turn, the influence of motors in creating a torsion on the bundles may be investigated. 


\subsection{Effects of geometrical confinement of MT-motor mixtures}

Eukaryotic cells exhibit diversity in their functions as well as their shape/size, ranging from red blood cells (of size $\sim 5 \mu \mathrm{m}$ ) to neurons (of size $\sim 100 \mu \mathrm{m}$ ). All the cytoskeletal polymers within these cells are constantly growing and shrinking by addition and loss of protein subunits at the same time as they are serving as tracks for molecular motors.

Drawing inspiration from these behaviours, we investigate the effects of confinement of the MT bundle-motor networks dealt within Section 3.1. The geometrical confinements in our case are in terms of volumes of the imaging sample chambers, namely chambers of heights 10 and $100 \mu \mathrm{m}$. Additionally, we observed an interesting behavioural change when the MT polymerization environments are varied. The main aim of this study was to experimentally investigate the influence of these geometries on the self-assembly and morphology of the MT networks under the influence of motor proteins.

The experimental samples were (i) MT-motor mixtures polymerized in Eppendorf tubes (tubes hereafter) of volume $0.5 \mathrm{ml}$ and then transferred to imaging chambers and (ii) MT-motor mixtures that are polymerized directly in the imaging chamber itself, that have volumes of $\sim 0.7 \mu \mathrm{l}$ and $\sim 7 \mu \mathrm{l}$ for $10 \mu \mathrm{m}$ and $100 \mu \mathrm{m}$ chambers respectively. In a previous study, a combination of various experimental parameters was found to correspond to maximum network speeds [42]. Based on this, we choose a final tubulin concentration of $0.6 \mathrm{mg} / \mathrm{ml}$, a PEG concentration of $0.6 \%(\mathrm{w} / \mathrm{v})$ and an ATP concentration of $1 \mathrm{mM}$ for all our experimental cases. All the samples described below were imaged at a $20 \times$ resolution under the confocal microscope to visualize an area corresponding to $635 \times 635 \mu \mathrm{m}^{2}$.

Additionally, to track the dynamics of MT bundles, we incorporated passive tracers of diameter $2 \mu \mathrm{m}$ (Refer Section 2.5). The tracer particles are attached to the MT backbone. Their trajectories offer an indirect method of tracking the MT bundle movement as a result of motor cluster activities. By quantifying the bead trajectories, we could characterize the spatio-temporal characteristics of the motor cluster-induced MT networks.

As the environment containing the tracers is quite complex owing to relatively high MT bundle densities and collective behaviour of MTs owing to individual motor protein dynamics, a common tool to assess transport at the temporal scale is the time-averaged mean squared displacement (MSD). For the total time measurements, we generally cut-off the number of time points at $1 / 4$ th the value of the total time to obtain reliable values for the ensemble MSD [81]. The MSD is usually a power-law 
function and is calculated using:

$$
<\vec{r}^{2}(\Delta)>_{t}=\frac{1}{T-\Delta} \int_{0}^{T-\Delta} d t[\vec{r}(t+\Delta)-\vec{r}(t)]^{2}
$$

where $\vec{r}(t)=(x(t), y(t))$ denotes the position vectors of the beads, $\Delta$ denotes the lag time in seconds and $\mathrm{T}$ is the overall measurement time (1/4th of the total time).

Thus, the four cases generated that we shall refer to and explain separately are listed below:

- $10 \mu \mathrm{m}$ tube-polymerized

- $10 \mu \mathrm{m}$ chamber-polymerized

- $100 \mu \mathrm{m}$ tube-polymerized

- $100 \mu \mathrm{m}$ chamber-polymerized

\subsection{1 $10 \mu \mathrm{m}$ tube-polymerized}

Firstly, we deal with MT-motor mixtures polymerized in a tube and visualized in a $10 \mu \mathrm{m}$ high chamber. The sample chamber is completely sealed to avoid artefacts due to flow. We can also visualize the fluorescent beads simultaneously in the field of the MT bundles. We visualized the samples for $30 \mathrm{~min}$ and observed a transition through three different regimes.

A typical experiment begins with an initial phase (0-15 min) where there is a disordered movement of the MT bundles in random directions as observed in Figure 3.11(A). We label it as an isotropic phase as characterized by the wide range of orientation distributions seen in Figure 3.11(D). Immediately after this period, there is a sudden collective motion towards a partially dominant orientation. This phase is labelled as the transient phase (Figure 3.11(B)). It is characterized by a momentary collective pause of the tracers followed by a swift transition towards a partially dominant orientation as depicted in Figure 3.11(E). The transient phase lasts for an average period of 5-8 min (with dominant angles around $-50^{\circ}$ and $50^{\circ}$ ) and then progresses towards a dominant orientation of MT bundles. Additionally, the tracers begin to move in a directed fashion and further orient the bundles in a single dominant direction, characterized by the sharp peak at $20^{\circ}$ in the orientation distribution (Figure 3.11(F)). We term this period as the directed phase. We should, however, note that this global orientational change occurs in different directions for every experiment.

These interesting transitions in the dynamics of MT-motor mixtures that cause the redistribution of the bundles collectively in a specific direction can be further 

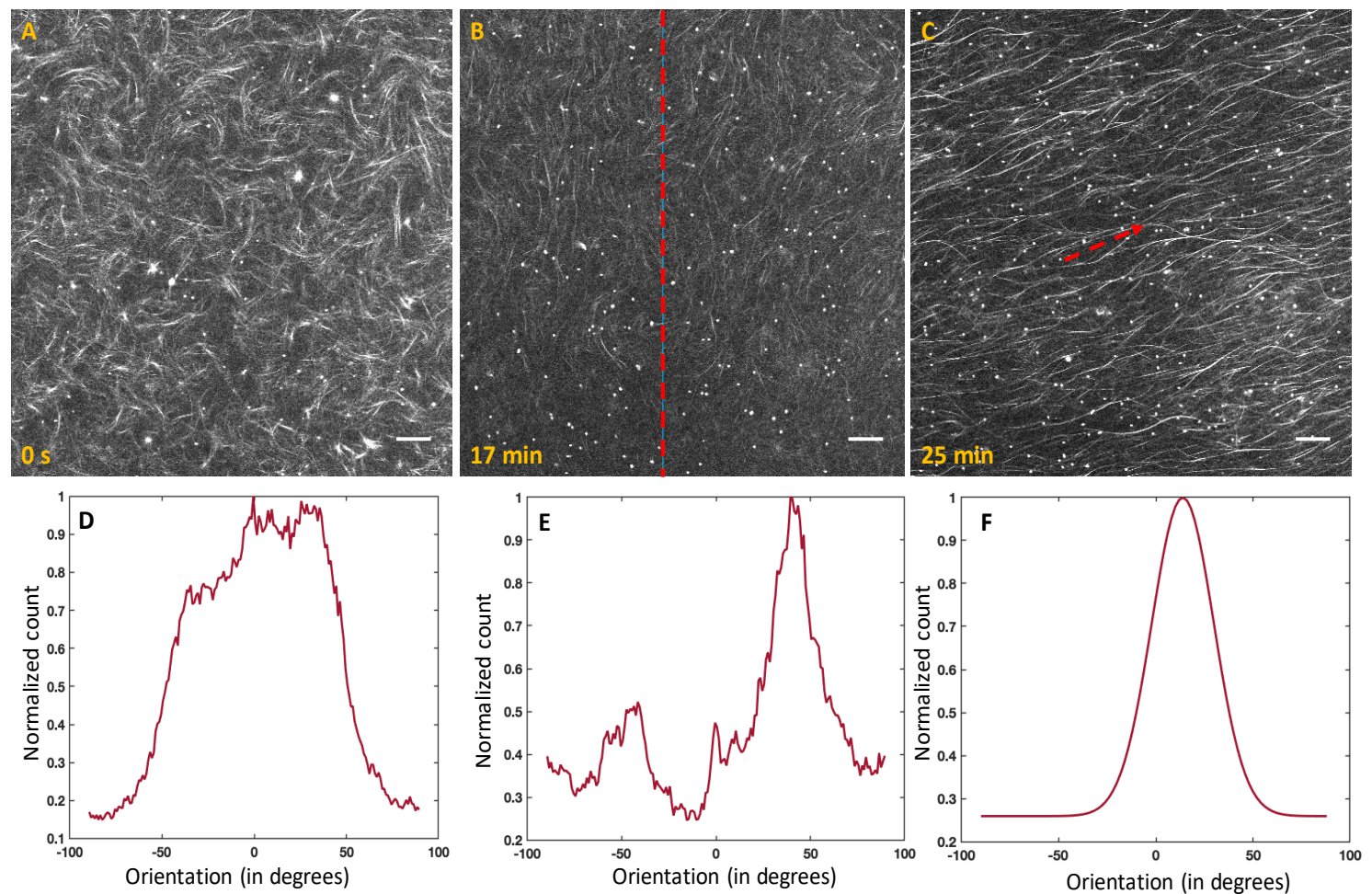

Figure 3.11: The phases of isotropic, transient and directed motion observed in the $10 \mu \mathrm{m}$ chamber and their corresponding MT bundle orientation distributions. Scalebar : $20 \mu \mathrm{m}$

quantified through the introduction of the nematic order parameter, $S$. As a measure of the orientation degree of the MT bundles, $S$ was calculated using the equation:

$$
S=\frac{1}{N_{M T}} \sqrt{\left(\sum_{\theta=-90}^{+90} R_{\theta} \cos 2 \theta\right)^{2}+\left(\sum_{\theta=-90}^{+90} R_{\theta} \sin 2 \theta\right)^{2}}
$$

where $N_{M T}$ refers to the total number of MT bundles in the frame, $\theta$ is the angle between every MT and the reference $\mathrm{x}$-axis that can have values between $90^{\circ}$ and $+90^{\circ}$, and $R_{\theta}$ is the frequency of every orientational angle $\theta$ of individual MT bundles. The orientations of the MT bundles were calculated using an ImageJ plugin called OrientationJ [82] and angular distributions were plotted as seen in Figure 3.12. Using this program, the images are sliced into quadrants and hence, the range of $\theta$ was between $-90^{\circ}$ and $+90^{\circ}$. These values were further used to determine $S$.

To characterize the temporal dynamics, we calculated the MSDs of individual particle trajectories. As the bundles move in 3D, when the tracers disappear and reappear at subsequent time points, they qualify as a separate trajectory. By estimating the MSDs of the particles, we can quantify them as power-law functions.

During the first isotropic phase, the calculated, averaged MSD curves from the 


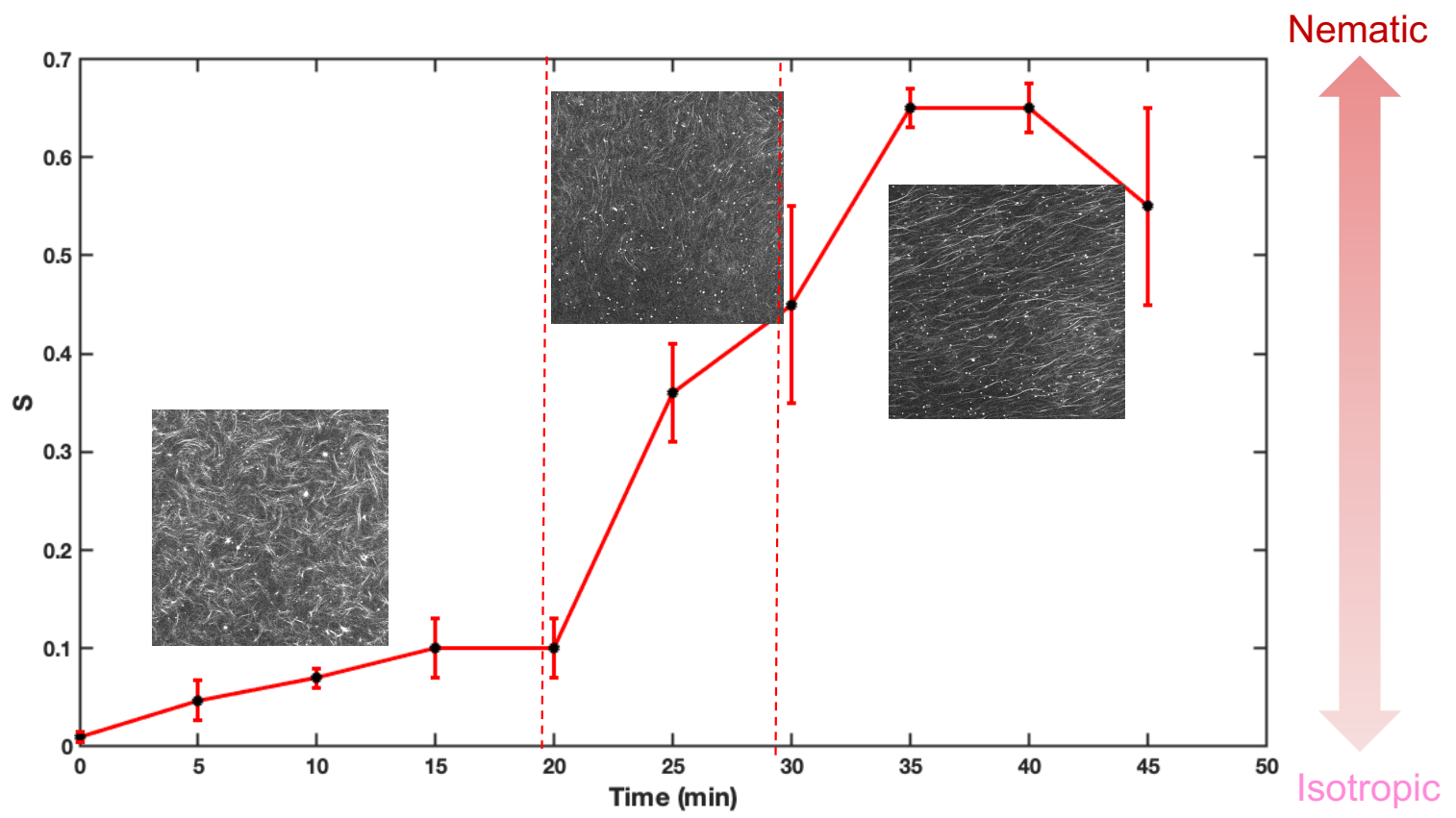

Figure 3.12: The increase in the value of the nematic order parameter, $S$ with time depending on the three phases of MT-motor behavior. $S$ varies between an isotropic value of 0.05 to a nematic value of 0.65 .

bead trajectories were found to have a $\Delta t^{1.48}$ power-law dependence. Although the particles exhibit a wide range of displacements owing to some sub-diffusive and super-diffusive behaviour as a result of activity, the mean value of this timedependence followed a value that lies between the diffusive and super-diffusive regime (0.1-1.8). When the transition occurred into the next phase, the MSD was found to have a $\Delta t^{1.52}$ dependence. Further, the trajectories in the directed phase were found to have a $\Delta t^{2}$ dependence (Figure 3.13 ).

Studies predict that the MSD curves at even longer timescales will usually turn diffusive due to exhaustion of ATP [42]. However, we cannot track beads for such long times and hence, this regime cannot be explored. For this reason, we divide the timescales of the three regimes by considering them as three different activities. It is essential to note that the three different regimes correspond to three regions of the chamber as long-time imaging of one region induces photobleaching. However, multiple areas of the chamber were checked to verify global behaviour. The variation in MSD dependence is a dynamic indication of the changes in the phases observed. An important morphological property that we consider when employing two polymerization modes in our setup is the length distribution of the MT bundles. Although the filaments continuously oscillate across multiple planes, we consider the bundles that stay within a plane during a particular time point and estimate their apparent length through a manual tracking method in ImageJ. The average length 

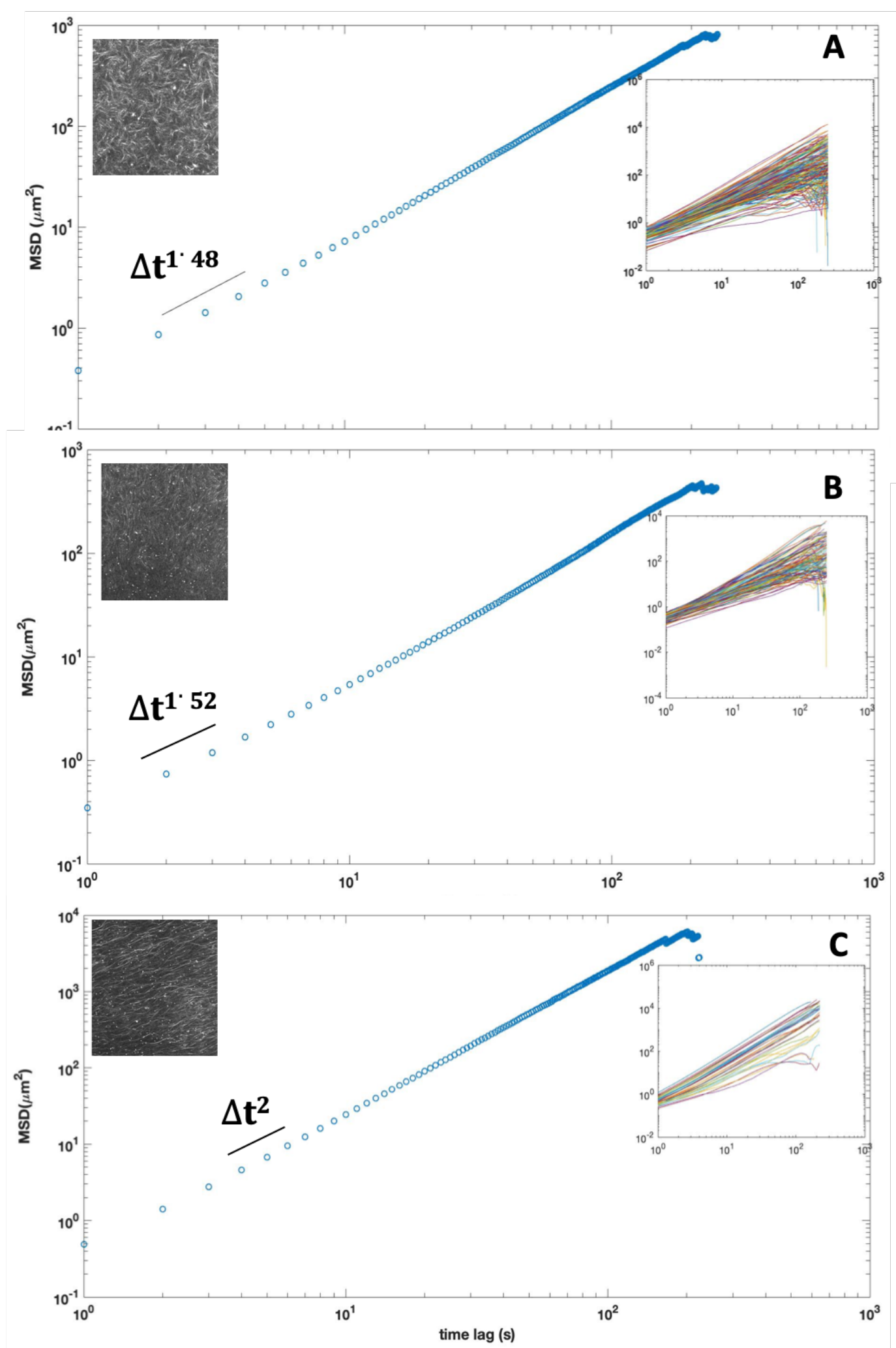

Figure 3.13: The time-averaged MSD behaviour corresponding to the three phases. The graphs inset depict the MSD paths of multiple trajectories that reflect their heterogeneous behaviour. 


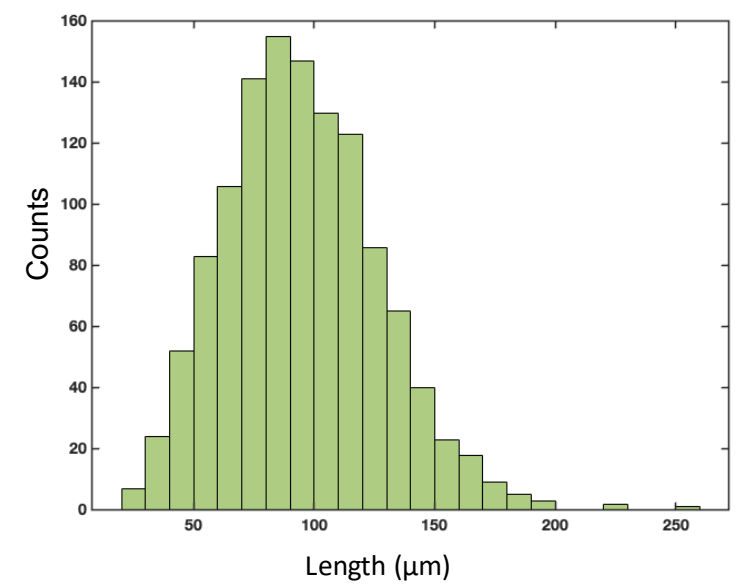

Figure 3.14: Length distribution of the tube-polymerized bundles and visualized in a $10 \mu \mathrm{m}$ chamber

was found to be $95 \pm 32 \mu \mathrm{m}$ (Figure 3.14).

\subsection{2 $10 \mu \mathrm{m}$ chamber-polymerized}

When an MT-motor mixture is directly polymerized in the sample chamber and visualized, the MT bundles typically are not visible due to low contrast. It is interesting that although the experimental conditions and parameters are the same, the MT bundles appear thinner. As we do not have access to this information, we hypothesize that the pre-conditioned confinement in the chamber may have influenced the number of MTs within a bundle. Additionally, the MT bundles may not orient themselves in a particular orientation but most of the trajectories follow a directed path as depicted in Figure 3.15(B). Moreover, when imaged over a long time (60

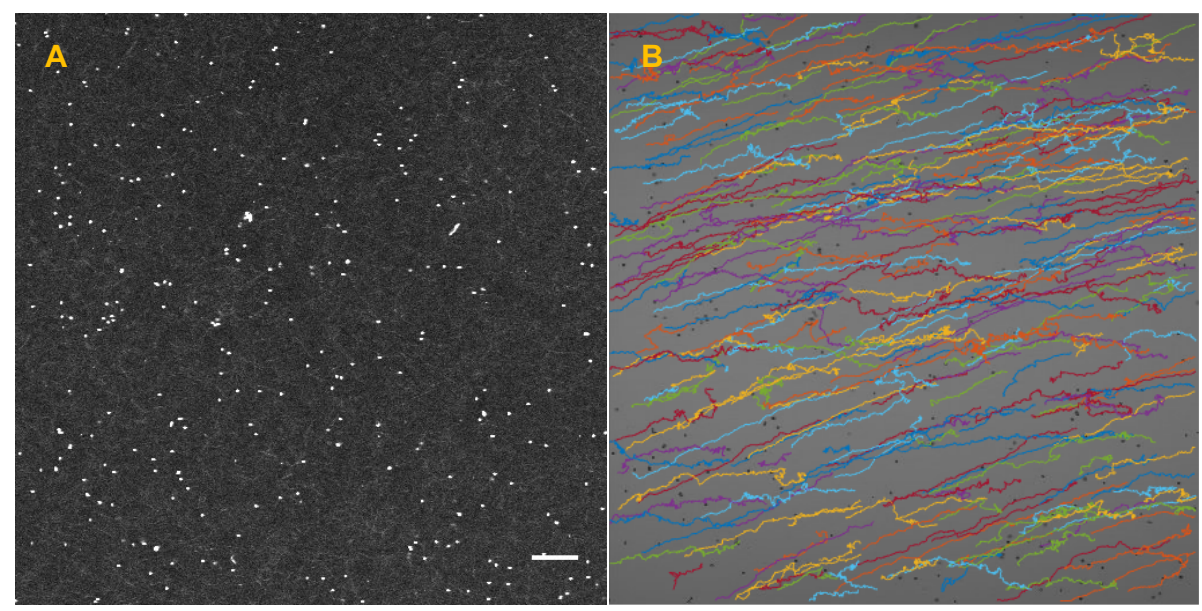

Figure 3.15: Particle trajectories (B) of the tracers that move in a $10 \mu \mathrm{m}$ chambers (A). Scale bar : $20 \mu \mathrm{m}$

min or more in different chamber regions), we observed a gradual decrease in the 
time-lag dependence of the MSDs. We observed the transition from a $\Delta t^{1.88}$ to a $\Delta t^{1.2}$ dependence (Figure 3.16). The length distributions of the MT bundles were
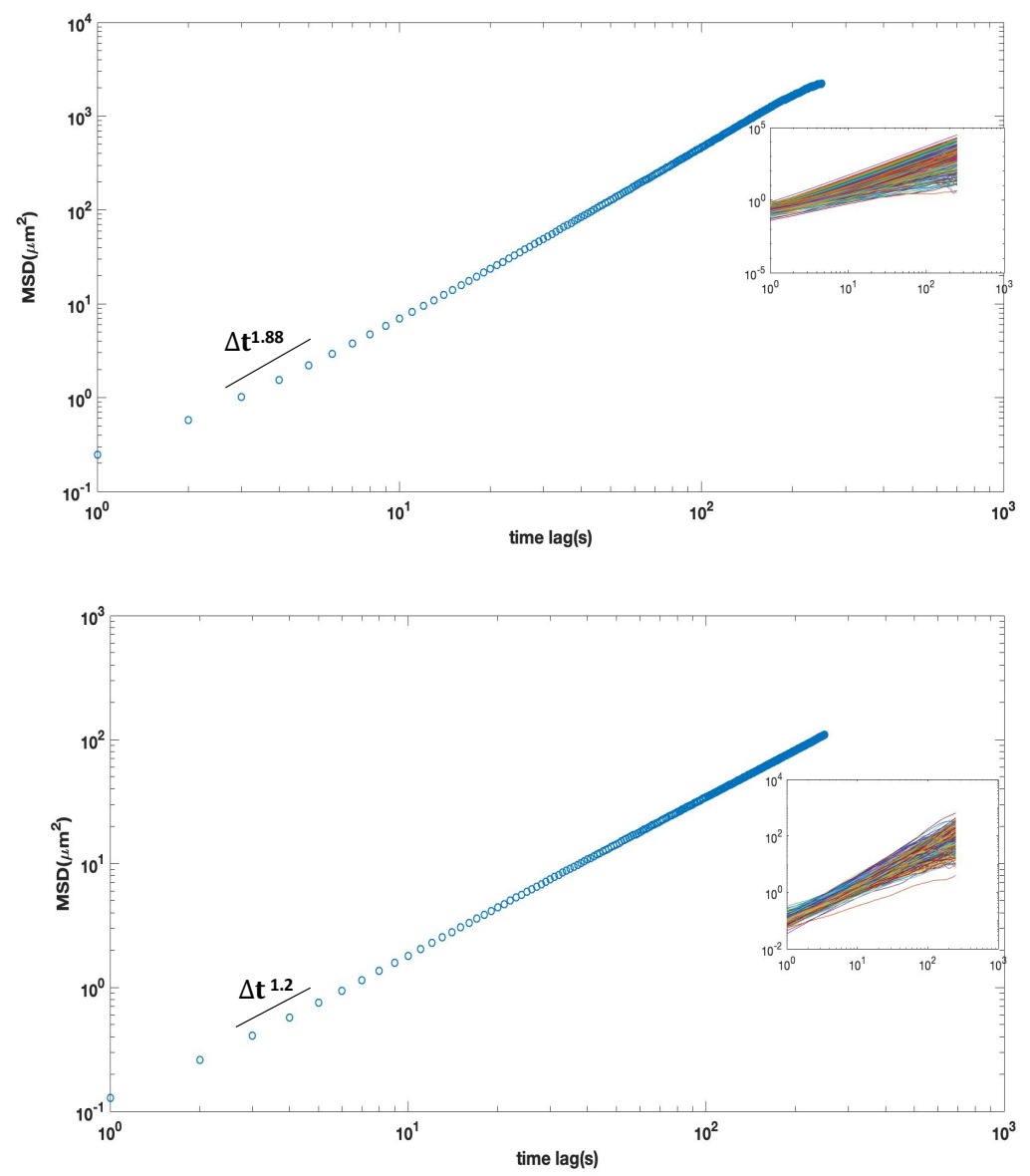

Figure 3.16: MSD plots of MT-motor dynamics for bundles polymerized in a $10 \mu \mathrm{m}$ chamber. Scale bar : $20 \mu \mathrm{m}$

estimated by tracking sample images visualized using a $60 \times$ magnification. The average length was found to be $17 \pm 4 \mu \mathrm{m}$.

Comparing the two cases of polymerized MT bundles in two different modes of polymerization gives us an idea regarding the tunability of the self-assembling process as well as the dynamics of the generated bundles. While MT bundles polymerized in a tube and transferred into a chamber exhibit three distinct phases of behaviour that grow to exhibit sub-diffusive behaviour, the bundles polymerized in the chamber directly transit into a diffusive state behaviour. The reason for this different behaviour could be related to the length scale of the bundles itself. The longer bundles cause motor clusters to travel longer paths before dissociating or interacting with an adjoining bundle. On the other hand, the short bundles could cause the quick dissociation of motor clusters along their length and cause them to traverse linear tracks and exhibit diffusive behaviour at longer scales. 


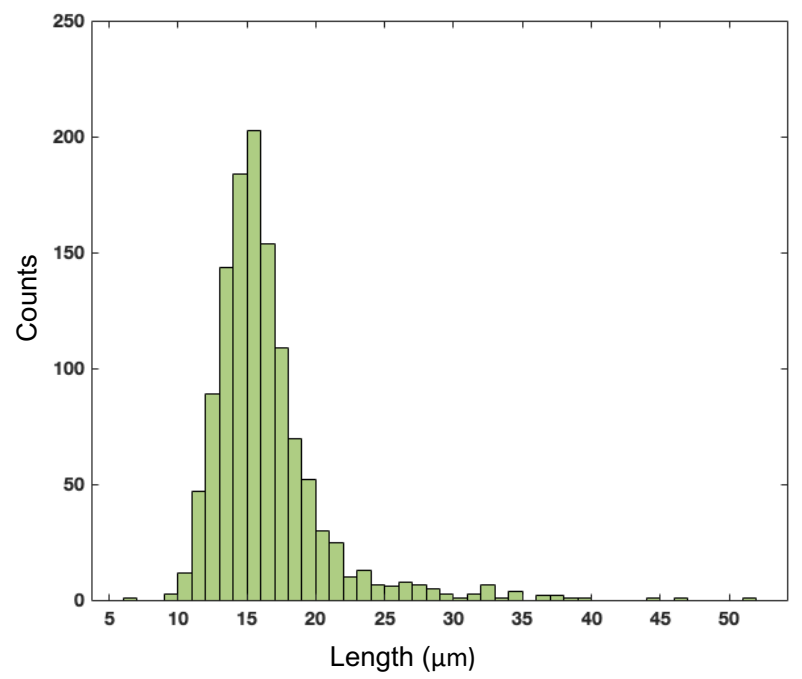

Figure 3.17: Length distribution of MT bundles polymerized in a $10 \mu \mathrm{m}$ chamber.

\subsection{3 $100 \mu \mathrm{m}$ tube-polymerized}

In this section, we shall deal with MT-motor mixtures in $100 \mu \mathrm{m}$ sample chambers that exhibit a characteristic extensile flow. The features of the MT bundles in 100 $\mu \mathrm{m}$ confinements are distinctly different from the $10 \mu \mathrm{m}$ samples. The experiments revealed the formation of an autonomous flow pattern that occured along multiple focal planes along the height of the chamber (Figure 3.18). An important feature of our observations was the fast, repeated cascades of extension, buckling, fracturing and self-healing of the constituent bundles. The larger bundle lengths could be due
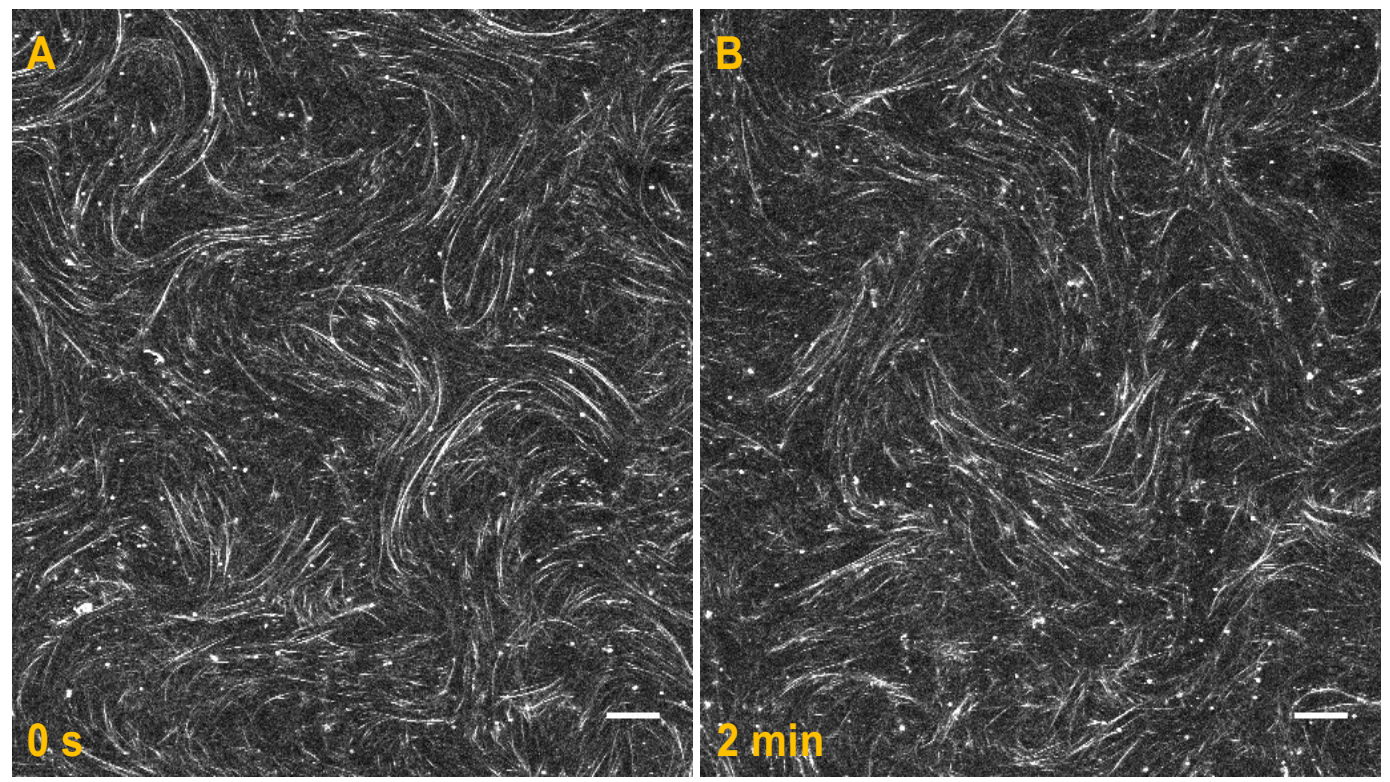

Figure 3.18: A short time elapse exhibits distinctive behaviour of the self-generated mixing in MT-motor bundles in $100 \mu \mathrm{m}$ chambers. Scale bar : $20 \mu \mathrm{m}$ 
to their constant re-healing in the background. The continuous 3-D movement of MT-motor bundles reveals a wide distribution in their length ranges. The average length of the MT bundles was found to be $147 \pm 35 \mu \mathrm{m}$ as seen in Figure 3.19. As the bundles continuously anneal and reheal, for the most part we consider their lengths when they are completely visible on one plane.

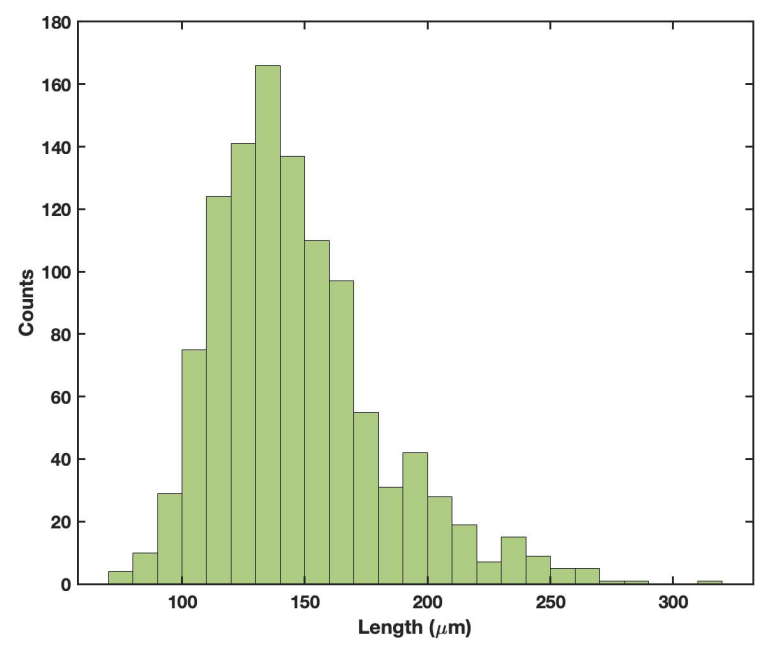

Figure 3.19: Length distribution of MT bundles polymerized in a tube and visualized in a $100 \mu \mathrm{m}$ chamber.
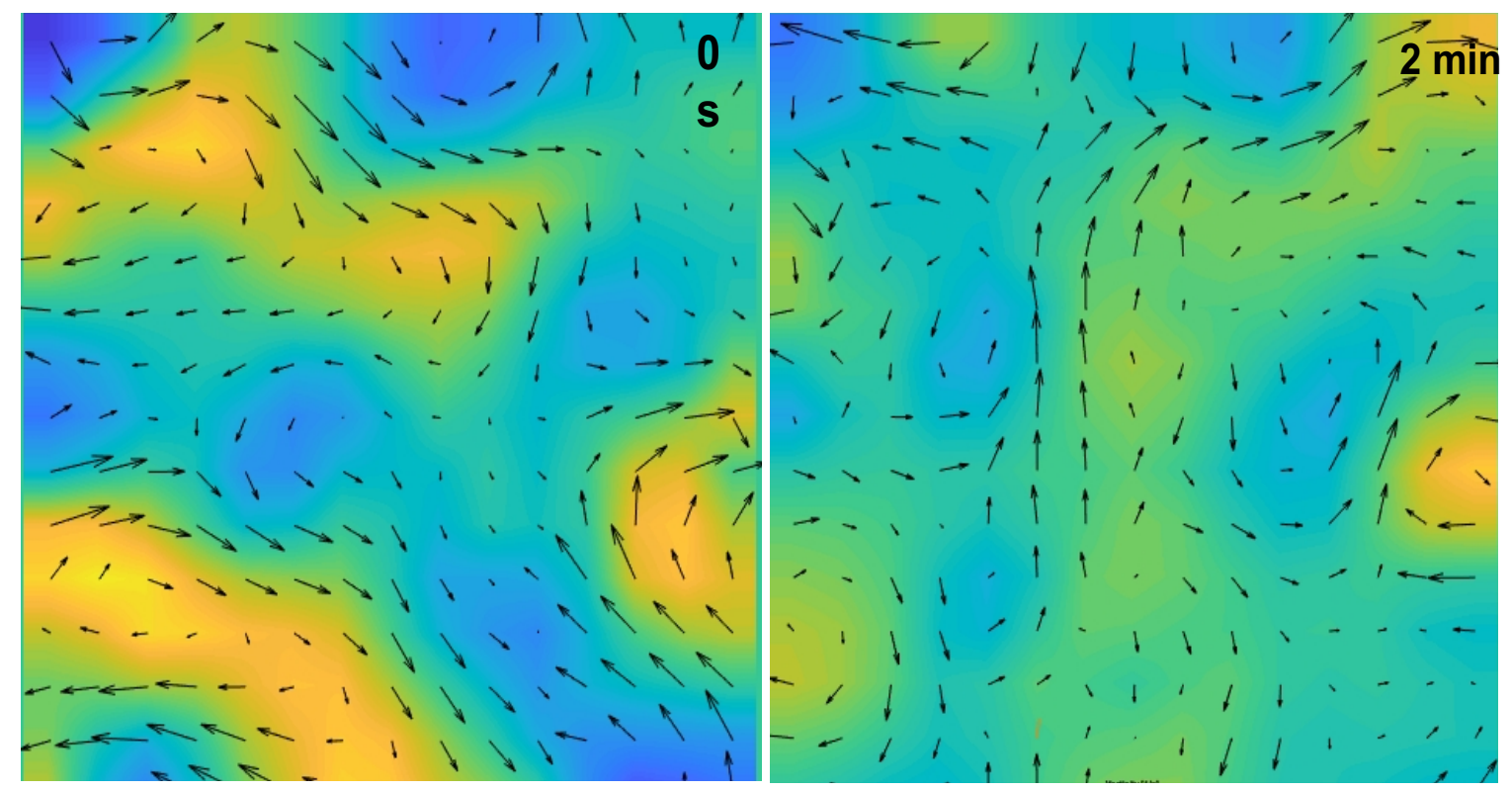

Figure 3.20: Particle imaging velocimetry (PIV) maps corresponding to the time points depicted in Figure 3.18

To characterize the dynamics of the behaviour for this particular case, we track the particles through the Particle imaging Velocimetry (PIV) technique to generate 
velocity fields of the moving particles (Figure 3.20). Vortices of varying intensities that continuously appear and disappear for short periods are a distinct feature observed in this experiment. Vorticity is defined as the local rotation of a fluid, mathematically calculated as the curl of the local velocity field. The yellow regions of the maps indicate points of highest vorticity which last typically for nearly $10 \mathrm{~s}$, having values between -0.6 and 0.6 per s.

\subsection{4 $100 \mu \mathrm{m}$ chamber-polymerized}

This last experimental variation deals with the polymerization of MT bundles in a $100 \mu \mathrm{m}$ sample chamber. It is interesting to note that the bundles exhibit a similar turbulent flow, but at a smaller scale (Figure 3.21). The MT bundle movements exhibit vorticities in the range -0.1 to 0.1 per $\mathrm{s}$, that reverse in the direction of their rotation nearly every $20 \mathrm{~s}$.

As seen in the scenario of the $10 \mu \mathrm{m}$ chamber-polymerized case, the apparent lengths of the MT bundles here are considerably smaller than the tube-polymerized mixtures. The average length was found to be $57 \pm 9 \mu \mathrm{m}$.

From the experimental studies described in this section, we observed how the self-assembly and dynamics of MT-motor bundles are affected under confinement. The well-characterized parameter so far was the length distributions which exhibit a wide range dependent on both the polymerization environment and confinement height.

Coupled with this, we found the length scale of the vorticities to be dependent on the polymerization environment. Vortexes generated in the $100 \mu \mathrm{m}$ tubepolymerized case are much greater in diameter when compared to the $100 \mu \mathrm{m}$ chamber-polymerized case. This difference could be possibly related to a buckling length scale of the constituent bundles or an intrinsic phenomena of the motors. Further PIV investigations in this direction could help in explaining this behavior. However, in this experimental work, we restrict the results to a qualitative description of our observations. 

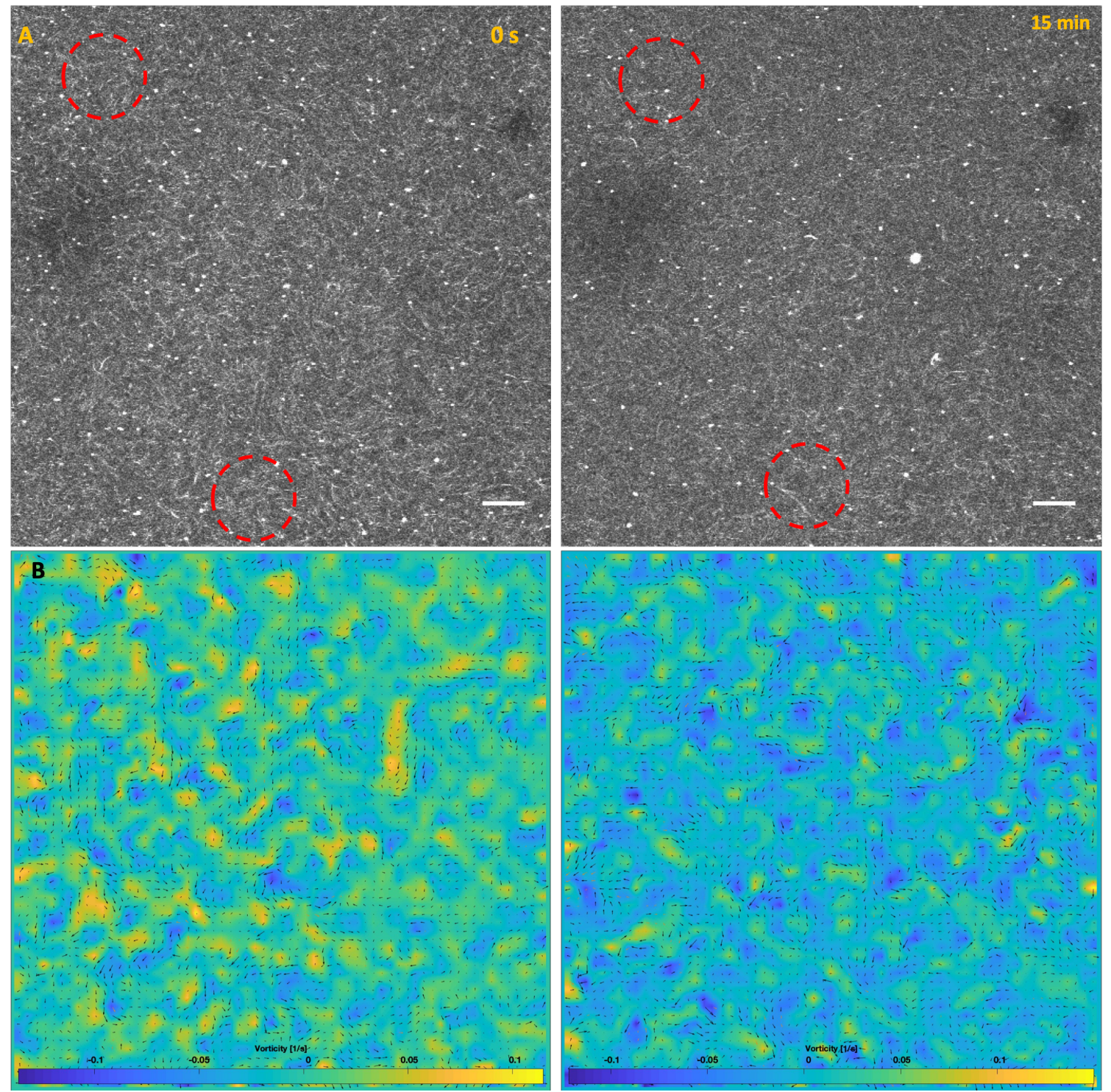

Figure 3.21: Time-lapse images of MT bundles in a $100 \mu \mathrm{m}$ chamber in panel A. Panel B shows their corresponding PIV maps indicating vorticities in both a clockwise and anti-clockwise direction. 


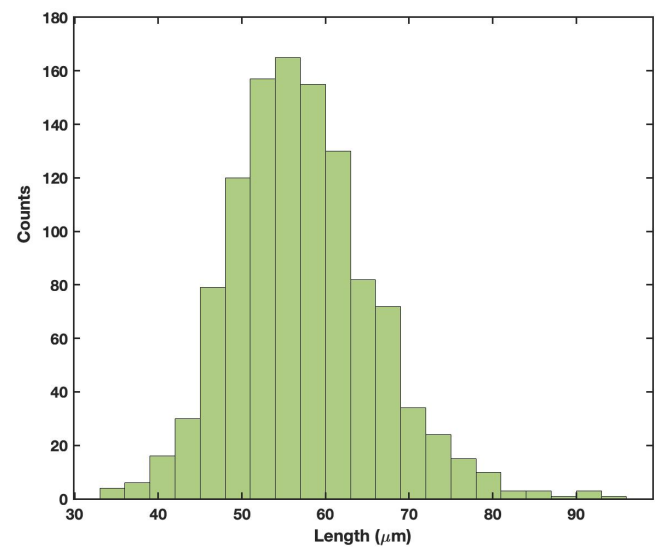

Figure 3.22: Length distribution of MT bundles polymerized in $100 \mu \mathrm{m}$ chamber

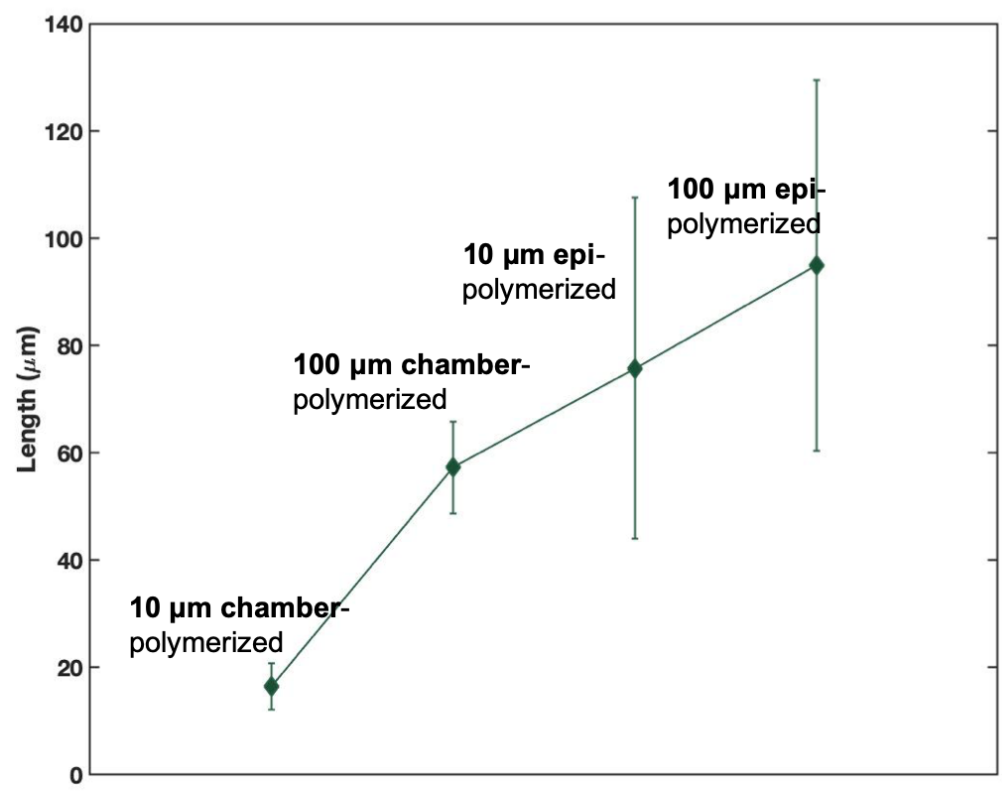

Figure 3.23: Comparison of average MT bundle lengths in the various configurations

\subsection{Oscillatory behavior of a single microtubule}

So far, we've dealt with higher density MT networks and their associated bundle dynamics. As the most basic approach, we look into single filaments created by a larger extent of dilution of MT-motor protein mixtures.

Why are single filament studies important? In nature, the transport of fluids at the micrometre to millimetre scale as well as the propulsion of microorganisms and sperms rely on cilia and flagella. Being a complex structure containing at least 600 different proteins [77] makes it extremely challenging to deduce the exact roles of the constituent proteins. As ciliary systems can be considered as operating out of equilibrium, its activity is the result of components rearranging due to intrinsic stochasticity. Thus, building a synthetic molecular system containing components 
that rearrange and reorganize continuously (in the presence of energy sources) could enable a better understanding of these molecular interactions.

In our approach, we present a minimal system comprising a single MT with a fixed end and a small number of motor proteins, that perform a continuous flagella-like beating in the presence of ATP. Compressive forces exerted by kinesin motors were found to cause buckling instabilities of these clamped MTs.

\subsubsection{Experimental results}

In our experimental setup, we make use of diluted polymerized MTs ( $\sim 8 \mu \mathrm{m}$ long $)$ decorated with motor clusters i.e. biotinylated kinesin clusters using streptavidin (see Section 2.4). We also make use of a non-ionic polymer, PLL-PEG to prevent irreversible protein adsorption on the surface [57]. However, we could occasionally observe some MTs with one tip clamped (usually $2 \mu \mathrm{m}$ ) to the surface (1-2 samples in every experiment) as illustrated in Figure 3.24.

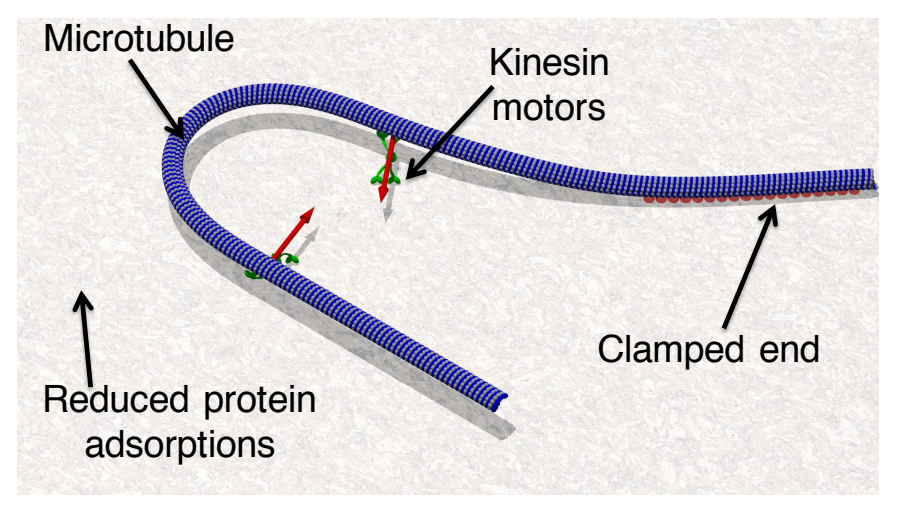

Figure 3.24: Schematic representation of the minimal system. It depicts a single filament clamped at one end, beating and buckling under the action of motor proteins interacting with the PLL-PEG functionalized surface [104]

This is likely due to the electrostatic interaction with the polylysine backbone of the PLL-PEG monolayer brush on the surface. The anchor point does not exhibit any rotational or translational degree of freedom. The free part of the MT exhibited oscillatory motion for time intervals up to $5 \mathrm{~min}$ as shown in Figure 3.25 . This motion can be attributed to the motors simultaneously bound on one end to the MT and another end at the surface, due to their organization in clusters. There is a kind of wave propagation that occurs from the bound toward the free end of the filament. This system can be described as follows:

(i) the kinesin motors in the clusters simultaneously bind either to the microtubules or unspecifically to the surface.

(ii) The motors exert forces directed along the tangential direction on the MT. 

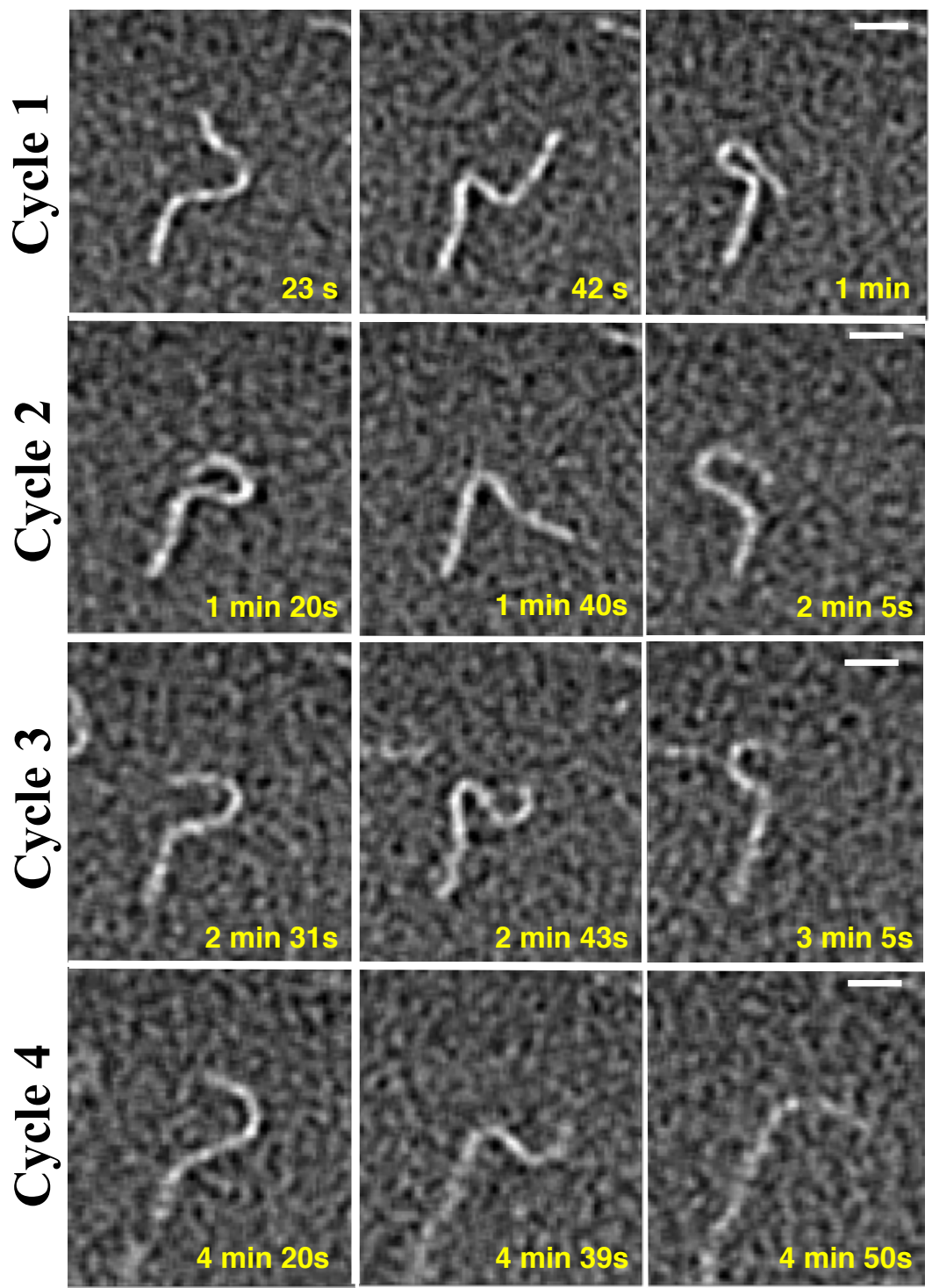

Figure 3.25: Oscillation cycles of a single MT clamped on a surface over time starting at $\mathrm{t}=0$. Scale bar: $2 \mu \mathrm{m}[104]$

(iii) The force that bends the filament has a longitudinal as well as a perpendicular component that allows it to arrive back at its original configuration. This snapping back of the MT can be due to (a) the MT detaching from the motors or due to (b) the motors breaking the bond with the PLL-PEG surface and being pulled away while it remains attached to the MT. Both these possibilities lead to a subsequent buckling when the filament reaches a new position.

The observed oscillations can be divided into cycles (horizontal panels in Figure 3.25 ), defined as the time needed by the filament to move from one portion of space to the adjacent portion and to come back. As the beating of our filaments was observed to be quasi-2D, we analyze their movement pattern by tracking the filament shape with the help of Jfilament [94, exemplary tracks are depicted in Figure 3.26(A). 
We characterized the tracked filament shape by a tangent angle $\psi(s, t)$, the angle
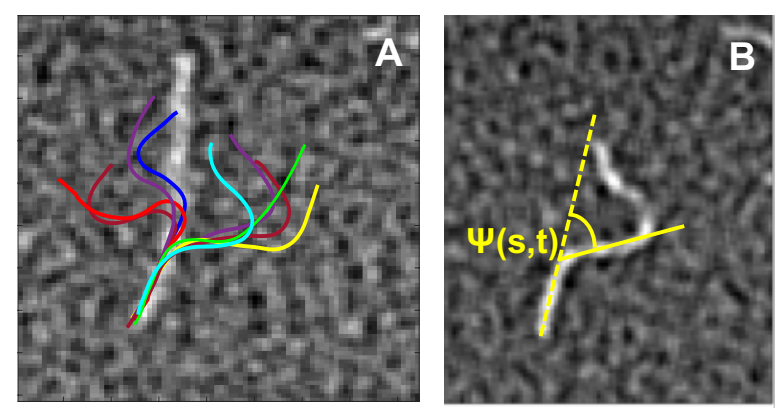

Figure 3.26: (A) The time evolution of oscillations can be tracked as seen in various colors. (B) the tangent angle $\psi(s, t)$ as a function of the arc length $s$ along the filament 104.

between the straight line representing the initial position of the filament and the local tangent angle of the arc length $s$ along the filament at a timepoint $t$ with $0 \leq s \leq L$, where $L$ is the length of the filament portion free to move, seen in Figure 3.26 (B). The shape changes were tracked up to 300 frames (5 mins imaging time) to obtain a kymograph that represents the periodicity of the filament's beating (Figure 3.27 .

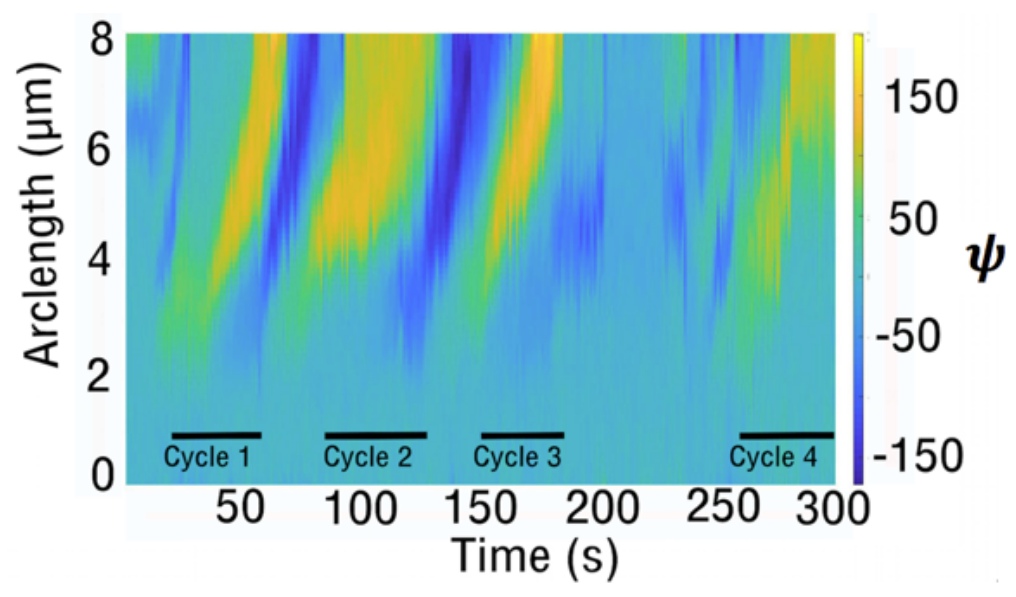

Figure 3.27: Kymograph of the tangent angle over time along the filament corresponds to the cycles pointed out in Figure 3.25 [104].

The observed pattern in Figure 3.27 represents a wave-like motion propagating from one end of the filament along its entire length, which can be understood as follows : After the first buckling instability, the resulting bend is subjected to continued forcing from the direction of the free end, which makes it bulge out further. As more filament is entering the bend, its position on the arc length automatically moves toward the free end. At the same time, each bend leads to a counter-bend with opposite curvature at the clamped end, that eventually increases in size and moves 
toward the free end, as the original bend did. The result is a periodic undulatory motion.

Although this beating profile may reflect a beating similar to eukaryotic flagella, it is important to note some essential differences in the two systems. In our experiments, the filaments interact with motors attached to a surface, and the motor forces thereby have the nature of force monopoles. However, dyneins in cilia and flagella act between axonemal MT doublets and therefore appear as force dipoles.
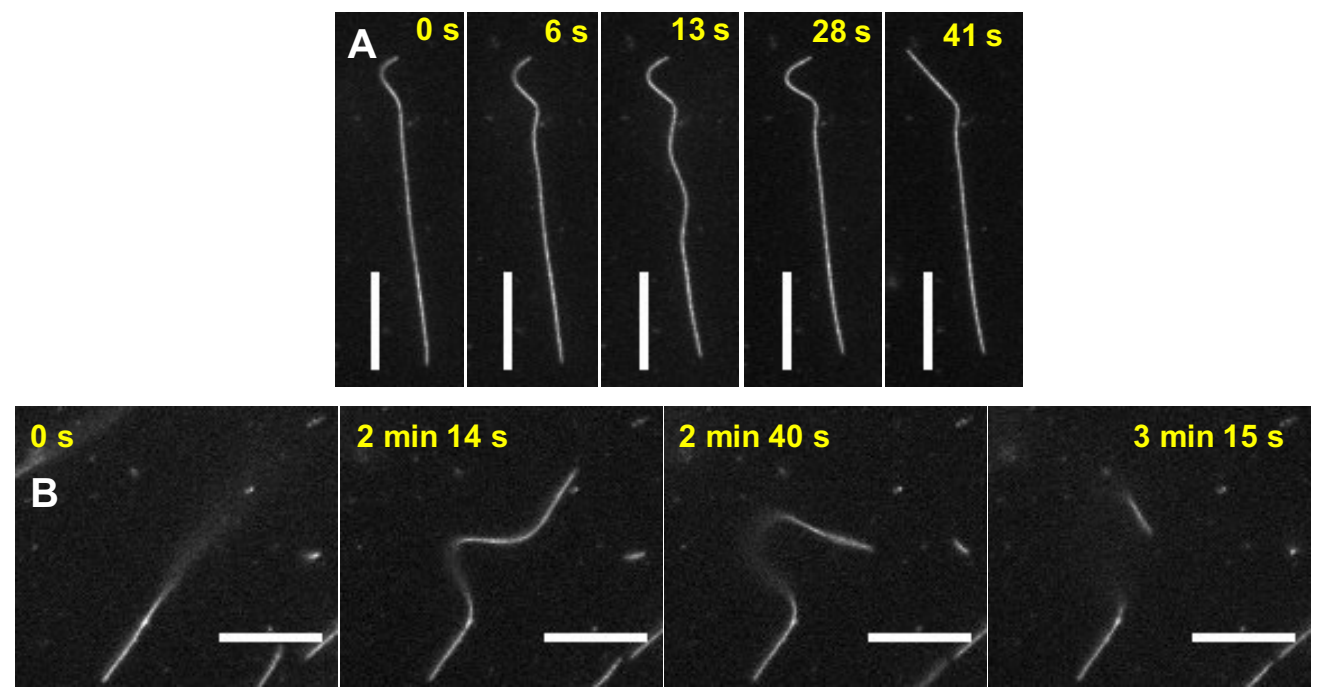

Figure 3.28: (A) Single filament with motors at a 100-fold higher concentration shows a reduced capacity to buckle continuously. (B) Single filament with motors at 2-fold lower concentration showing reduced buckling and out-of-plane movement. Scale bar : $2 \mu \mathrm{m}$

Control experiments performed without a PLL-PEG surface functionalization did not show any buckling filaments, revealing that the unspecific binding of both motor proteins and MTs onto a surface does not allow any filament to move freely. To estimate the motor density range over which filament buckling can occur, we repeated experiments by employing a different motor concentration. While using a 100-fold higher concentration, no continuous oscillations were observed (Figure 3.28 (A)).

The buckling events were less frequent and limited in time compared to the case presented in Figure 3.25. Again, by reducing the motor concentration 2-fold, the buckling events were also less frequent and limited in time. The sliding of the filaments was followed by the filaments leaving the focal plane due to the missing anchor point, as seen in Figure 3.28(B).

For the case of this clamped filament, we can also define an active bending length according to the definition introduced in Section 5.1.2. While the mean length of the MTs, in this case, was found to be $6.7 \pm 0.6 \mu \mathrm{m}$ with $\sim 2 \mu \mathrm{m}$ of the filament 
pinned on the surface, we found that the time-dependent active bending length $\mathfrak{b}$ was $3.4 \pm 1.4 \mu \mathrm{m}$.

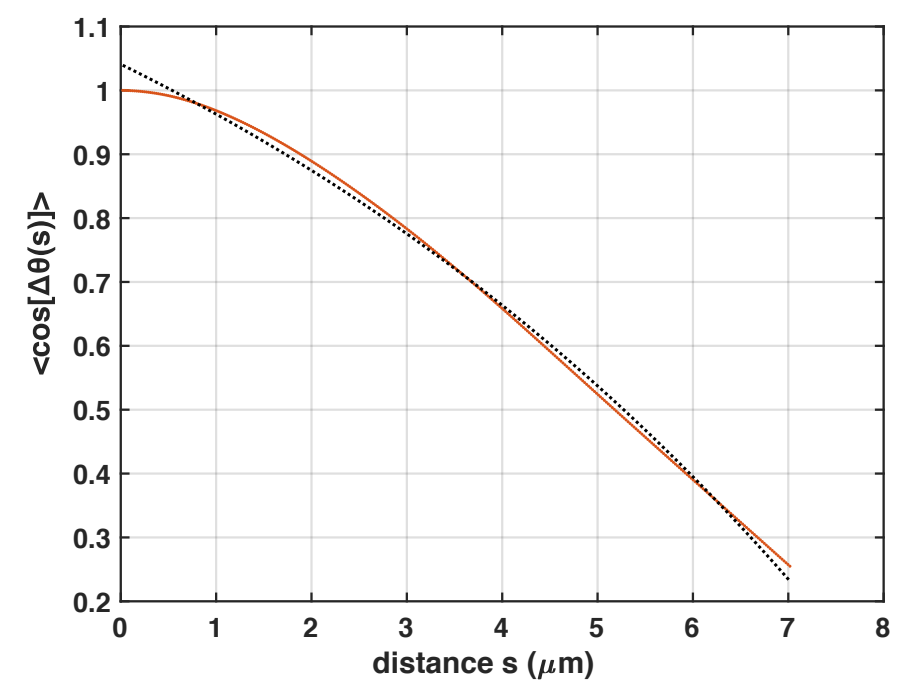

Figure 3.29: The tangent-tangent correlation of the oscillating filament with data (in red) and exponential fit (in black).

\subsubsection{Theoretical discussion}

To further verify the motor concentration requirements to buckle the filament, theoretical calculations were performed in the Department of Prof. Dr. Ramin Golestanian (Living Matter Physics Department, Max Planck Institute for Dynamics and Self-Organization, Göttingen).

If we consider a filament of length $\mathrm{L}$ attached to motor clusters (pinning points) with an average spacing $\lambda$ and assume it is torque-free at the motor positions, the force needed to buckle the segment is given by its Euler buckling modes:

$$
F_{B}=\frac{\pi^{2} E I}{\lambda^{2}}
$$

From equation 3.5, as the buckling length,$\lambda$ decreases, the force required to buckle the segment increases. As the distance between the points of zero inflection (zero bending moment) decreases, the segment shows an increased resistance to buckling. This denotes that the highest value of the compressive force exerted by the motor ensemble is in the segment right next to the clamped end. It corresponds to $F_{M} L / \lambda$, $F_{M}$ being the stall force of a motor cluster. The two forces become equal at a critical length

$$
L_{c}=\frac{\pi^{2} E I}{F_{M} \lambda}
$$

$L_{c}$ is inversely proportional to the motor spacing, hence proportional to the density. Assuming $F_{M}=8 \mathrm{pN}$ (maximum force produced by a single motor), $E I=0.4$ 
$\times 10^{-23} \mathrm{Nm}^{2}$ (calculated from experiments of thermally bending MTs) and $\lambda=1$ $\mu \mathrm{m}$, we obtain $L_{c}=5 \mu \mathrm{m}$. A filament of $5 \mu \mathrm{m}$ length will generally buckle if the number of motors acting on it is between 1-5. The distribution of motor forces that explains the observed shapes could be determined using a maximum likelihood approach.(Figure 3.30)

The tangential components of the forces are in the range $-8 \mathrm{pN}$ and $0 \mathrm{pN}$, a value consistent with forces due to single motors. The negative sign denotes forces that push the filament toward the clamped end. The normal components of the forces as well are in the range -10 to $10 \mathrm{pN}$, corresponding to 1-2 kinesins.
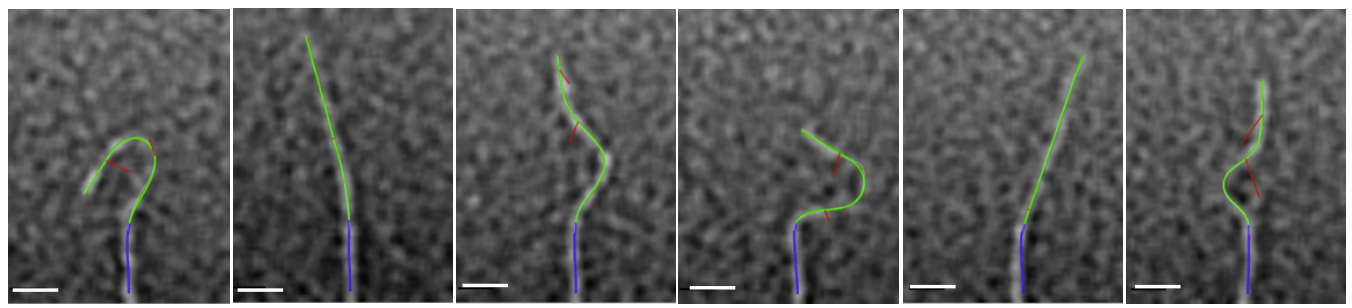

Figure 3.30: The reconstruction of the filament shape with the corresponding force points (in red) [104].

\subsection{Surface attachment of MTs}

In the scenario described above, the clamping of the filament to a protein-repellent surface occurs due to a probable electrostatic attraction between the MT and the surface. However, to increase the probability of attaching MTs to a surface in a global manner, we adopt two techniques. These are based on using nucleation templates along with short MTs (seeds) to increase the nucleation rate of MTs that can eventually grow on the surface. The procedures are described in detail in Section 2.7 .

As preliminary experiments to attach MTs, we made use of micropillars (of diameter $5 \mu \mathrm{m}$ ) and an anchor system such as MAP (microtubule-associated protein) for binding MTs on the surface.

Note that we polymerized only single MTs in the absence of motor proteins. Their average length was found to be $13 \pm 4 \mu \mathrm{m}$. The disadvantage of this method was that the attachment of MAP on pillars was not always verifiable and there was a large density of MTs attached on the untreated surface.

An alternative method for MT attachment was based on micropatterning techniques. The micropatterns used were dots of diameter $5 \mu \mathrm{m}$, stripes and squares of width $10 \mu \mathrm{m}$ each. By oxidising a layer of silane - PEG on a glass surface to generate patterns, we made use of a neutravidin coating as a template to attach 


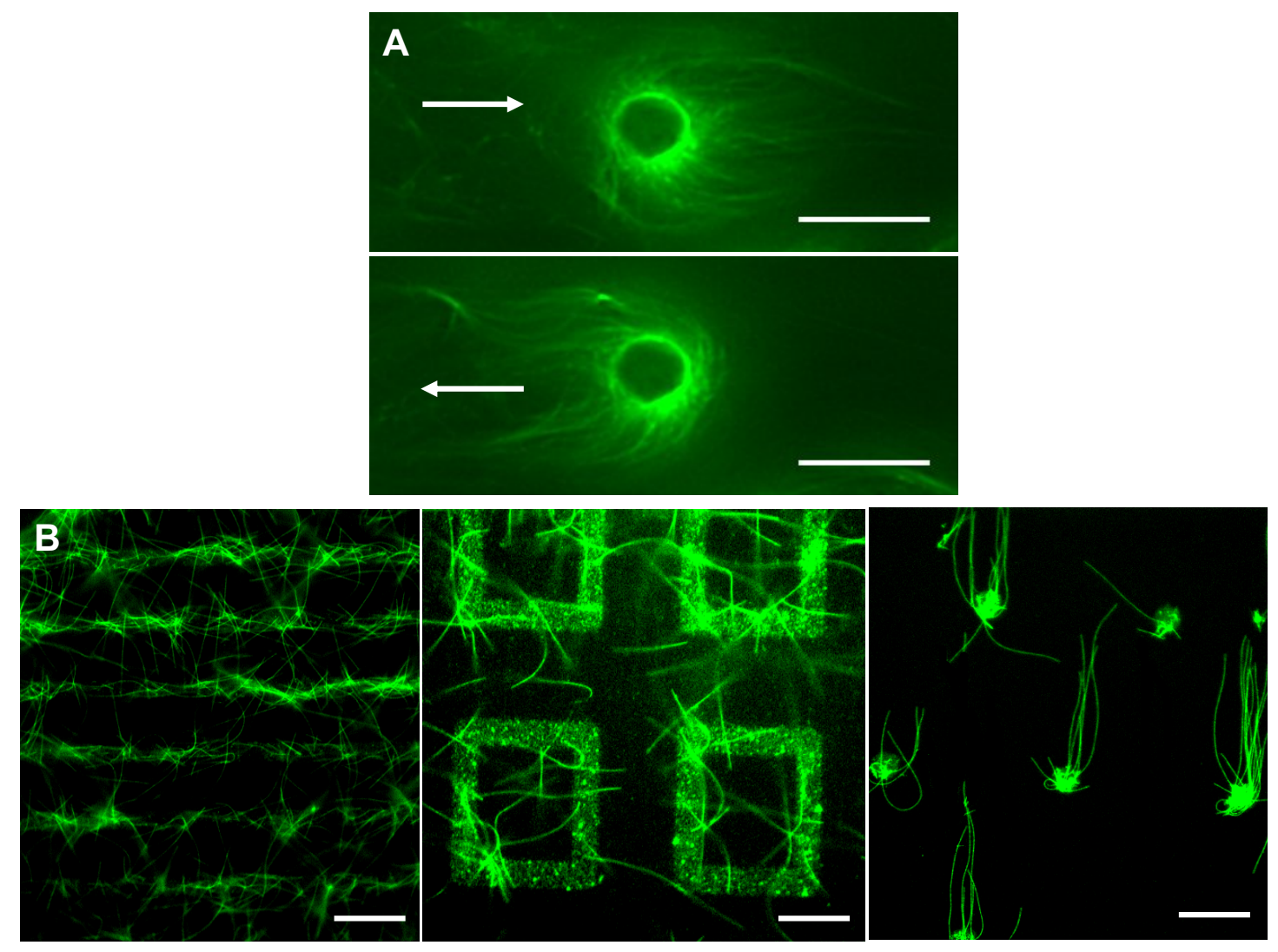

Figure 3.31: (A) MTs attached via MAP on micropillars. Arrows indicate the direction of applied flow. Scale bar: $10 \mu \mathrm{m}$ (B) Microtubules attached via biotinneutravidin on stripe, square and dot patterns. Scale bar : $10 \mu \mathrm{m}$

biotinylated MT seeds and elongate MTs. This method was found to be robust due to a double passivation treatment of the surface (using both silane - PEG and PLL - PEG) that lead to very few randomly attached MTs. Global formation and attachment of microtubules over large scales of patterns could be observed with an average length of $16.4 \pm 1.3 \mu \mathrm{m}$.

The next steps in this direction would involve incorporation of motors into this setup in a systematic method to observe and understand the collective behaviour of microtubules attached on a surface using nucleation templates. This could generate results that differ from the case of a single microtubule and offer insights into how motor dynamics may be influenced in a global patterned repetition of microtubules, in contrast to randomly oriented microtubule bundles. 


\section{Chapter 4}

\section{Discussion}

In this work, we investigated MT-motor protein systems at various levels of complexity. We established their similarities and differences concerning natural biological phenomena such as coordinated ciliary beating and intracellular streaming. The synthetic reconstitution of such complex active systems offered us insights into the behaviours of the individual building blocks and their interactions that generated interesting dynamics. In turn, we investigated their self-assembling behavior from the level of network organization down to the level of active single filaments.

In this section, we shall highlight our interpretation of the results of these systems that were presented in Chapter 3.

\subsection{Motor cluster behaviour in networks}

In our experiments, we generated motor protein clusters that were labelled using streptavidin. These clusters were embedded within dense MT networks. To broadly obtain ideas of motility of these clusters and their activity generation in these networks, we proposed a simple tracking method (Section 3.1).

The interesting factors in our observations were the distinct 'jumps' made by the clusters when they reached either the end of a bundle or a neighbouring MT-bound cluster. Populations of motor protein clusters were divided into two types: clusters that persistently moved within a small area of active bundles and clusters that quickly translocated across multiple bundles through long-distance jumps. These jumps also occurred mid-way during its path run, depending on the availability of a neighbouring, sliding bundle. During this time instant, the observed cluster velocity was nearly $1 \mu \mathrm{ms}^{-1}$ instead of the average active velocity of $0.26 \mu \mathrm{ms}^{-1}$. The velocity is in agreement with that of single kinesins on PEG-depleted bundles obtained by Conway et al [16]. Also, our experiments confirmed the observations of Furuta et al, regarding the cooperativity between multiple motors [28]. The velocities remain 
unaffected by the number of motors when they are closely spaced in the form of clusters. We also confirmed the effect of PEG on the slowing down of cluster motility from $0.8 \mu \mathrm{ms}^{-1}$ (in the absence of PEG) to $0.26 \mu \mathrm{ms}^{-1}$. The slowing down of the clusters could be interpreted as an effect of an increased viscosity environment or increased inter-filament friction due to the extensile sliding of one bundle against another.

Another interesting observation of the motor cluster motility is their enhanced run lengths. We redefined run lengths in terms of association (on single bundles) and activity lengths (on multiple bundles), both found to have values of $\sim 4 \mu \mathrm{m}$. These similar values indicated that a cluster travels the same average distance before being dissociated from one bundle and becoming active on another bundle. The values of run lengths we obtained are far greater than those reported on single filaments $(600 \mathrm{~nm})$ [102]. The enhanced run lengths can be attributed to the cluster configuration of motors and availability of long MT bundles as tracks, which lowers the probability of cluster dissociation at shorter lengths. The high value of association time that we obtained (corresponding to 21s) agrees with previous studies that describe longer times of residence of kinesin in the presence of neighbouring motors [84]. Additionally, this value was found to be greater than association times previously reported (100 ms) [102]. Future experiments dealing with motor protein cluster-MT dynamics can be designed to distinguish their separate dynamics for a more precise calculation of association and activity parameters.

A large population of the clusters exhibited periods of pause at least once during their entire run on the bundles. This is in agreement with previous studies where the number of motors that pause on bundles $(57 \%)$ is higher compared to single MTs (43\%) [16]. The cooperativity between multiple motors also results in the pause of one motor protein within a cluster being affected by its neighbors within a cluster. In other words, the probability of a cluster unbinding from an MT bundle is lower, as long as all the motor proteins within the cluster do not dissociate from the bundle. It reflects in the high value of pause time of $4.5 \mathrm{~s}$ that we observed. This ability of the cluster to wait without detaching from a bundle reflects its ability to work even under crowded conditions.

More recent studies by Seitz et al explained the processive movement of single kinesins on crowded MTs using a labelling agent such as quantum dots [90. It was revealed that properties such as run-length and dwell time are insensitive to the crowded MT environment. However, we should consider the fact that the crowded MTs in their case was a few (4-5) single filament MTs suspended in a gliding assay. To our knowledge, cluster dynamics in highly crowded conditions have not been reported before.

In the experiments described here, we should note that the activity and associa- 
tion parameters pertaining to the kinesin clusters do not distinguish the movement of the MT bundles. The values obtained here are a characterization of the combined dynamics of bulk motor-MT bundles. Further analysis that distinguishes the dynamics of kinesin clusters could provide us a better understanding of the observed differences in the motor cluster activity and association.

The MT-motor protein cluster networks contain randomly distributed MT bundles of mixed polarities. The bundles may be oriented in an anti-parallel or parallel fashion. Depending on this, the motor clusters may cause the bundles to slide against each other or use them as tracks to walk until their ends. Such heterogeneous organizations of networks determine the bending and buckling behaviour of these bundles that contain multiple MTs as their constituents.

\subsection{Bundled active networks}

The components towards building MT-motor networks contain MTs, motor complexes and PEG as the main components. This method of organizing the building blocks to generate active bundles was pioneered in the works by Sanchez et al [89, 88] as described in the introduction. The interactivity between these components through depletion and motor-induced forces results in the dynamic behaviour of the MT bundles.

Adopting a similar approach, we diluted the dense active networks to obtain individual bundles free to move in 3D. They exhibited a variety of motions: continuous bending and sliding into multiple planes, buckling and some reversible cycles of merging and disassembly.

Recent in silico studies have characterized the dynamics of active self-propulsion of individual filaments on a surface [52, 53] as well as dense layers [80] through the distinction of their behaviour into the polymer and spiral regimes of activity. The introduction of a flexure number as the ratio of a filament's activity to its bending rigidity helped in understanding the regime to which they belong.

Since the bundles in our experiments are diluted, we cannot segregate them into regimes due to their low densities. Instead, we quantified their bending dynamics by individually tracking them. As an analogy to persistence length due to thermal fluctuations, we defined a parameter called the active bending length, $\mathfrak{b}$, a characteristic length over which active bending occurs. It was interesting to observe that the merged bundles have a length range of $100-300 \mu \mathrm{m}$, but the active bending length was in the range $80-90 \mu \mathrm{m}$. It means that the bending occurs over a fixed length range, irrespective of the total bundle lengths. However, to confirm this bending behaviour, we can propose some control experiments. We can investigate the influence 
of changes in ATP and PEG concentrations on the characteristic lengths $\mathfrak{b}$. The very reason for the activities we observed are the competing extensile forces. Thus, further experiments in this direction can establish if individual bending activities can be varied by tuning experimental parameters. Furthermore, we must remember that the active bundles are free to move in 3D. More precise calculations of their bending activities are possible if visualized and tracked in 3D. A preliminary step was undertaken in this direction to visualize this behaviour through the multi-plane imaging technique described here.

In Section 3.3, we further considered dense active networks and subjected them to an external mechanical stimulus such as geometrical confinement. By confining active MT-motor mixtures in chambers of heights $10 \mu \mathrm{m}$ and $100 \mu \mathrm{m}$, we were able to observe significant changes in their self-assembly, organization and characteristic behaviour. The length distributions of MT bundles varied according to their polymerization environments. They were shorter $(20-60 \mu \mathrm{m})$ when polymerized in chambers, and longer when polymerized in tubes $(80-100 \mu \mathrm{m})$. Our results showcased the transition from a directed organization of bundles (in the $10 \mu \mathrm{m}$ setup) to a chaotic mixing state as the confinement heights were increased (in the 100 $\mu \mathrm{m}$ setup). The chaotic state was characterized by the continuous appearance and disappearance of large-scale and small-scale vortices, dependent on the polymerization environment. The vortices can be classified as weak and strong, depending on the period for which they last. Further PIV studies of this system are still under investigation in our group.

We found the case of the $10 \mu \mathrm{m}$ chamber confinement equally interesting due to phase transitions it exhibits. The system initially starts with an isotropic movement of the active network that gradually transitions into a nematic ordered phase over a period of $30 \mathrm{~min}$. It was further verified by calculations of the nematic order parameter $(S)$ that increased with increasing order. The final state of globally aligned bundles that we obtained is still a matter of further investigation. We hypothesize that the randomly moving bundles at the start of the imaging generate a flow due to the collective behaviour of the motors. These flows could be powerful enough to transform the orientations of the bundles on a larger scale. It can be considered to be similar to densely suspended rods confined in a box of small volume. Perturbations due to motor forces could align the rod-like structures in one direction at some timepoint. Another hypothesis is that a surface-attached layer of MT bundles may influence the bundles in neighbouring layers (which are not too many, considering the chamber height is $10 \mu \mathrm{m}$ ) to orient along its dominant direction.

This interesting change in behaviour can be used to draw analogies with cellular environments, their organization and reactions to mechanical stimuli such as wall friction. Cell morphology in vivo is usually characterized by heterogeneous, three- 
dimensional environments of different sizes. Geometrical confinements on the cell in the form of their different heights and the presence of cellular membranes are known to influence the cytoskeletal organization and intracellular streaming within them. Inside the cell, filament-motor interactions generate fluid flow in the cytoplasm, a process called cytoplasmic streaming. This phenomenon has been reported in Drosophila and Xenopus oocytes [68, 98, to have long-range influences on cytoplasmic organization and motion.

Thus, our experimental designs served as a way to represent similarities to cellular behaviour and interactions, as a single cell can hold a variety of geometries of biopolymer organizations within it. For example, cells can develop flat protrusions (such as lamellipodia) for cell motility as well as elongate into 3D spherical mitotic spindles for cell division.

\subsection{Single active filament behaviour}

The last biomimetic approach by using the basic building blocks consisted of a single clamped MT that exhibited persistent oscillations due to a random distribution of motors. Interestingly, this behaviour that caused up to four beating cycles of the MT was observed to be similar to flagella behaviour as depicted in the kymograph in Figure 3.27 .

This minimal system depicted a beating velocity of -400 to $0 \mathrm{nms}^{-1}$. The negative value indicates the filament moves towards the clamped end. Although these velocities are considerably lower than natural cilia that exhibit $10 \mu \mathrm{ms}^{-1}$, one should note that the motor density packing in cilia is much higher and coordinated (20 motors per $100 \mathrm{~nm}$ ). Our studies experimentally demonstrated an autonomous molecular system that dynamically self-organizes through its elasticity and the interaction with the active forces exerted by motor clusters. By considering a semiflexible polymer with one clamped end, subject to local drag, Sekimoto and coworkers demonstrated a dynamic instability that results in spontaneous rotation and flapping oscillations of actin driven by myosins in vitro [91]. Similarly, a study of the spiral formation and rotation about a fixed point was presented in [10].

Additionally, several theoretical studies have discussed filaments subjected to active tangential compressive forces. A phase diagram of tangentially propelled forces in [14] as a function of the filament length, passive elasticity, propulsion force and the noise was provided to carry out minimal experimental tests.

The essential difference between our system and these models is the fact that a small number of motors are involved.

A summary of the three experimental scenarios presented in this work can be 
seen in Figure 4.1 .

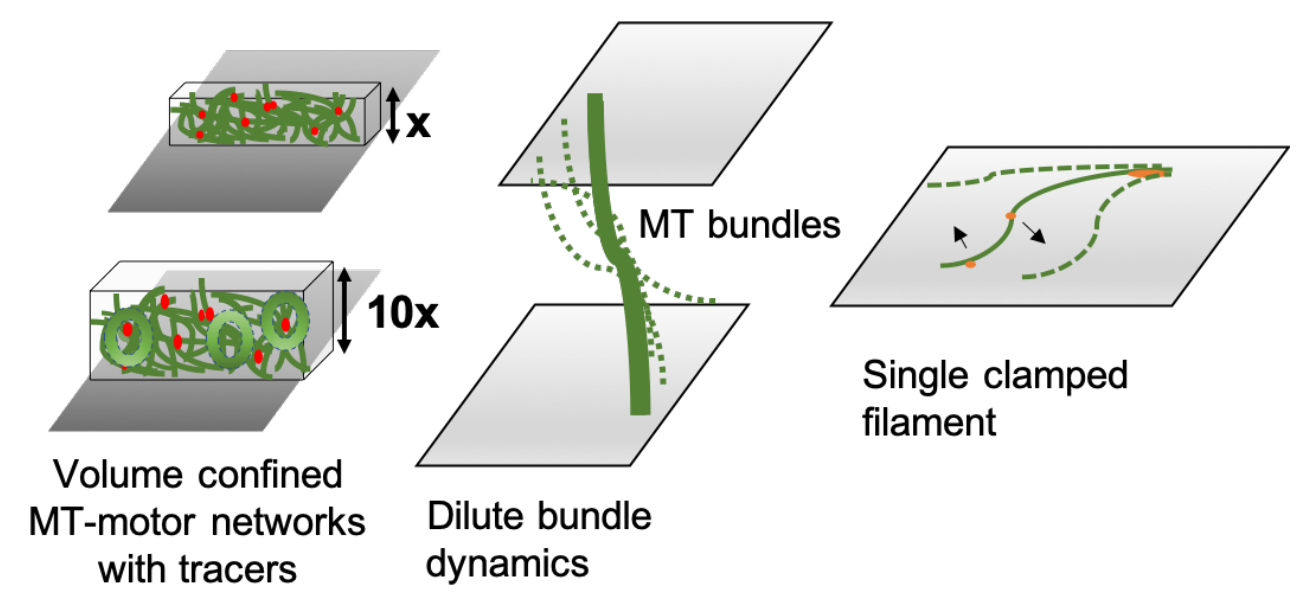

Figure 4.1: Schematic description of the geometrically confined networks, dilute bundles and single oscillating filament.

The exact mechanism of ciliary beating due to motors remains as nature's most enduring puzzle. Earlier theoretical models have predicted the occurrence of cilia like beating due to the self-organization of just a few essential components [11, 13]. They revealed beating patterns due to an interplay of the basal properties of the axoneme and mechanical feedback of the dynein motors [83, 64] and metachronal waves due to hydrodynamic coupling [39]. Hence, the current challenge is the bottom-up construction of synthetic cilia by using similar building blocks and design principles as those of biological systems.

\subsection{Towards synthetic reconstitution of cilia}

In the last decade, many efforts have been undertaken in facing the grand challenge of developing cilia-like microscopic actuators able to generate flow and mix fluids under the influence of different stimuli.

Artificial analogues of cilia were developed by using electrostatic fields, magnetic fields, and even light in an environment on a micrometre scale. Electrostatic cilia were actuated through voltage differences between a double-layered electrode that caused rapid oscillations [18]. The more widely-used approach of magnetic artificial cilia involved generation of 'conical beats' and self-assembled aligning under external magnetic fields [23, 24, 105]. More recent works include unidirectional propagation of chemical waves on a gradient of self-oscillating polymer brushes [66] and fabrication of polymer flow sensors using MEMS bundles of piezoelectric nature [6].

However, all these approaches have the drawback of missing the molecular constituents of natural cilia and do not offer any possibility to decipher the mechanism 
of spontaneous ciliary beating. The intriguing nature of biological ciliary fields is based on the coordination of thousands of motor proteins that results in beating patterns. Similarly, several thousands of these cilia are known to coordinate their beating patterns at the next hierarchical level.

Building upon previous works, we were able to generate dense active bundled networks that could be confined, diluted to obtain individual bundles and single beating filaments. Our system represented a minimal realization of bottom-up approaches towards understanding various cellular behaviours.

\subsection{Conclusion and Outlook}

In the direction of synthetically reconstituting ciliary behaviour, the next level of complexity would involve generating arrays of bundles that are equally spaced. To mimic the beating behaviour of cilia, the works of Sanchez et al [88, 89] made use of minimal components such as MTs, motor proteins and depletion agents to assemble coordinated structures. However, the exact nature of the coupling between the surface and beating bundles is not clearly understood. Further experimental steps would involve systematically improving clamping techniques to generate filaments fixed on one end. As a preliminary effort, we generated patterned surfaces with a nucleation-template based MT attachment technique that works on larger scales. The attached filaments are polarity-sorted at controlled distances and the experimental setup is quite robust. A possible method for the inclusion of motor proteins could be the incorporation of microfluidic methods onto the patterns. It could serve as a technique to get rid of the excess motor protein-MT aggregates to avoid dense network formation in the presence of a protein-repellent polymer brush on the surface.

When we view the bigger picture, the interior of a cell is crowded due to linked polymer networks and various organelles of different lipid compositions. Thus, to understand specific functions of various components (in our case, MT-kinesin behaviour), in vitro reconstitutions of these structures served as the most tractable solution. Arguably, the biochemical environment in our experiments is far away from the actual cell composition. The dynamical states are set by extrinsic parameters such as concentrations. Nonetheless, minimal synthetic reconstitutions can offer a deeper understanding of the basic physical and molecular mechanisms that control a range of behaviour such as cell motility, cargo and fluid transport.

In conclusion, the results presented in this thesis are summarized as follows :

- Kinesin clusters within MT networks generate active forces and exhibit enhanced behaviours (run length and association time) in comparison to single 
kinesins, thus giving us an idea of long-range transport in crowded environments.

- The individual bending dynamics of bundles in the presence of motor protein clusters exhibit a characteristic active bending length in a small range of 85$90 \mu \mathrm{m}$, irrespective of the resultant bundle lengths that vary between 100-300 $\mu \mathrm{m}$.

- Geometrically confined active networks exhibit a range of behaviours when chamber heights are increased; from a directed orientation with a nematic ordering to a disordered phase characterized by vorticities.

- A single clamped MT with a few randomly distributed motor proteins exhibits cilia-like beating. The behaviour is a combination of the intrinsic elasticity of the filament, the active forces exerted by the motor proteins and attachmentdetachment cycles between the constituent building blocks. 


\section{Bibliography}

[1] B. Afzelius, R. Dallai, S. Lanzavecchia, and P. Bellon. Flagellar structure in normal human spermatozoa and in spermatozoa that lack dynein arms. Tissue and Cell, 27(3):241-247, 1995.

[2] C. E. Aitken, R. A. Marshall, and J. D. Puglisi. An oxygen scavenging system for improvement of dye stability in single-molecule fluorescence experiments. Biophysical journal, 94(5):1826-1835, 2008.

[3] B. Alberts, A. Johnson, J. Lewis, M. Raff, K. Roberts, and P. Walter. Molecular biology of the cell 4th edn (new york: Garland science). Ann Bot, 91:401, 2002.

[4] C. Allen and G. G. Borisy. Structural polarity and directional growth of microtubules of chlamydomonas flagella. Journal of molecular biology, 90(2):381402, 1974.

[5] N. S. Allen and R. D. Allen. Cytoplasmic streaming in green plants. Annual review of biophysics and bioengineering, 7(1):497-526, 1978.

[6] M. Asadnia, A. G. P. Kottapalli, K. D. Karavitaki, M. E. Warkiani, J. Miao, D. P. Corey, and M. Triantafyllou. From biological cilia to artificial flow sensors: Biomimetic soft polymer nanosensors with high sensing performance. Scientific reports, 6:32955, 2016.

[7] A. Azioune, N. Carpi, Q. Tseng, M. Thery, and M. Piel. Protein micropatterns: A direct printing protocol using deep uvs. In Methods in cell biology, volume 97, pages 133-146. Elsevier, 2010.

[8] M. Bathe, C. Heussinger, M. M. Claessens, A. R. Bausch, and E. Frey. Cytoskeletal bundle mechanics. Biophysical journal, 94(8):2955-2964, 2008.

[9] S. M. Block, L. S. Goldstein, and B. J. Schnapp. Bead movement by single kinesin molecules studied with optical tweezers. Nature, 348(6299):348, 1990. 
[10] L. Bourdieu, T. Duke, M. Elowitz, D. Winkelmann, S. Leibler, and A. Libchaber. Spiral defects in motility assays: a measure of motor protein force. Physical review letters, 75(1):176, 1995.

[11] C. J. Brokaw. Molecular mechanism for oscillation in flagella and muscle. Proceedings of the National Academy of Sciences, 72(8):3102-3106, 1975.

[12] R. G. Burns. $\alpha-, \beta$-, and $\gamma$-tubulins: Sequence comparisons and structural constraints. Cell motility and the cytoskeleton, 20(3):181-189, 1991.

[13] S. Camalet, F. Jülicher, and J. Prost. Self-organized beating and swimming of internally driven filaments. Physical review letters, 82(7):1590, 1999.

[14] R. Chelakkot, A. Gopinath, L. Mahadevan, and M. F. Hagan. Flagellar dynamics of a connected chain of active, polar, brownian particles. Journal of The Royal Society Interface, 11(92):20130884, 2014.

[15] D. Chowdhury. Stochastic mechano-chemical kinetics of molecular motors: a multidisciplinary enterprise from a physicist's perspective. Physics Reports, 529(1):1-197, 2013.

[16] L. Conway, M. W. Gramlich, S. Ali Tabei, and J. L. Ross. Microtubule orientation and spacing within bundles is critical for long-range kinesin-1 motility. Cytoskeleton, 71(11):595-610, 2014.

[17] D. L. Coy, M. Wagenbach, and J. Howard. Kinesin takes one 8-nm step for each atp that it hydrolyzes. Journal of Biological Chemistry, 274(6):36673671, 1999.

[18] J. den Toonder, F. Bos, D. Broer, L. Filippini, M. Gillies, J. de Goede, T. Mol, M. Reijme, W. Talen, H. Wilderbeek, et al. Artificial cilia for active microfluidic mixing. Lab on a Chip, 8(4):533-541, 2008.

[19] A. Desai and T. J. Mitchison. Microtubule polymerization dynamics. Annual review of cell and developmental biology, 13(1):83-117, 1997.

[20] A. Doostmohammadi, J. Ignés-Mullol, J. M. Yeomans, and F. Sagués. Active nematics. Nature communications, 9(1):3246, 2018.

[21] E. H. Egelman. Comprehensive biophysics. Elsevier, 2012.

[22] H. P. Erickson. Microtubule surface lattice and subunit structure and observations on reassembly. The Journal of cell biology, 60(1):153-167, 1974. 
[23] F. Fahrni, M. W. Prins, and L. J. van IJzendoorn. Micro-fluidic actuation using magnetic artificial cilia. Lab on a Chip, 9(23):3413-3421, 2009.

[24] B. L. Fiser, A. R. Shields, M. Falvo, and R. Superfine. Highly responsive coreshell microactuator arrays for use in viscous and viscoelastic fluids. Journal of Micromechanics and Microengineering, 25(2):025004, 2015.

[25] P. M. Fordyce, M. T. Valentine, and S. M. Block. Advances in surface-based assays for single molecules. Single-molecule techniques: A laboratory manual.(Selvin, P. \& Ha, T. ed), pages 431-460, 2008.

[26] F. J. Fourniol, P. Bieling, R. D. Mullins, D. A. Fletcher, T. Surrey, et al. Micropattern-guided assembly of overlapping pairs of dynamic microtubules. In Methods in enzymology, volume 540, pages 339-360. Elsevier, 2014.

[27] D. S. Friedman and R. D. Vale. Single-molecule analysis of kinesin motility reveals regulation by the cargo-binding tail domain. Nature cell biology, 1(5):293, 1999.

[28] K. Furuta, A. Furuta, Y. Y. Toyoshima, M. Amino, K. Oiwa, and H. Kojima. Measuring collective transport by defined numbers of processive and nonprocessive kinesin motors. Proceedings of the National Academy of Sciences, 110(2):501-506, 2013.

[29] S. Ganguly, L. S. Williams, I. M. Palacios, and R. E. Goldstein. Cytoplasmic streaming in drosophila oocytes varies with kinesin activity and correlates with the microtubule cytoskeleton architecture. Proceedings of the National Academy of Sciences, 109(38):15109-15114, 2012.

[30] T. Gao, R. Blackwell, M. A. Glaser, M. D. Betterton, and M. J. Shelley. Multiscale polar theory of microtubule and motor-protein assemblies. Physical review letters, 114(4):048101, 2015.

[31] A. Gennerich and R. D. Vale. Walking the walk: how kinesin and dynein coordinate their steps. Current opinion in cell biology, 21(1):59-67, 2009.

[32] S. P. Gilbert and K. A. Johnson. Expression, purification, and characterization of the drosophila kinesin motor domain produced in escherichia coli. Biochemistry, 32(17):4677-4684, 1993.

[33] L. Giomi. Geometry and topology of turbulence in active nematics. Physical Review X, 5(3):031003, 2015. 
[34] L. Giomi, M. J. Bowick, X. Ma, and M. C. Marchetti. Defect annihilation and proliferation in active nematics. Physical review letters, 110(22):228101, 2013.

[35] F. Gittes, B. Mickey, J. Nettleton, and J. Howard. Flexural rigidity of microtubules and actin filaments measured from thermal fluctuations in shape. The Journal of cell biology, 120(4):923-934, 1993.

[36] R. E. Goldstein and J.-W. van de Meent. A physical perspective on cytoplasmic streaming. Interface focus, 5(4):20150030, 2015.

[37] P. Guillamat, J. Ignés-Mullol, and F. Sagués. Control of active liquid crystals with a magnetic field. Proceedings of the National Academy of Sciences, 113(20):5498-5502, 2016.

[38] P. Guillamat, J. Ignés-Mullol, and F. Sagués. Taming active turbulence with patterned soft interfaces. Nature communications, 8(1):564, 2017.

[39] B. Guirao and J.-F. Joanny. Spontaneous creation of macroscopic flow and metachronal waves in an array of cilia. Biophysical journal, 92(6):1900-1917, 2007.

[40] D. D. Hackney. Kinesin atpase: rate-limiting adp release. Proceedings of the National Academy of Sciences, 85(17):6314-6318, 1988.

[41] D. D. Hackney. Highly processive microtubule-stimulated atp hydrolysis by dimeric kinesin head domains. Nature, 377(6548):448, 1995.

[42] G. Henkin, S. J. DeCamp, D. T. Chen, T. Sanchez, and Z. Dogic. Tunable dynamics of microtubule-based active isotropic gels. Philosophical Transactions of the Royal Society A: Mathematical, Physical and Engineering Sciences, 372(2029):20140142, 2014.

[43] C. Heussinger, M. Bathe, and E. Frey. Statistical mechanics of semiflexible bundles of wormlike polymer chains. Physical review letters, 99(4):048101, 2007.

[44] F. Hilitski, A. R. Ward, L. Cajamarca, M. F. Hagan, G. M. Grason, and Z. Dogic. Measuring cohesion between macromolecular filaments one pair at a time: depletion-induced microtubule bundling. Physical review letters, 114(13):138102, 2015.

[45] J. Howard et al. Mechanics of motor proteins and the cytoskeleton. Sinauer associates Sunderland, MA, 2001. 
[46] J. Howard, A. Hudspeth, and R. Vale. Movement of microtubules by single kinesin molecules. Nature, 342(6246):154, 1989.

[47] J. Howard, A. J. Hunt, and S. Baek. Assay of microtubule movement driven by single kinesin molecules. In Methods in cell biology, volume 39, pages 137-147. Elsevier, 1993.

[48] W. Hua, E. C. Young, M. L. Fleming, and J. Gelles. Coupling of kinesin steps to atp hydrolysis. Nature, 388(6640):390, 1997.

[49] I. Ibañez-Tallon, N. Heintz, and H. Omran. To beat or not to beat: roles of cilia in development and disease. Human molecular genetics, 12(suppl_1):R27-R35, 2003.

[50] D. Inoue, B. Mahmot, A. M. R. Kabir, T. I. Farhana, K. Tokuraku, K. Sada, A. Konagaya, and A. Kakugo. Depletion force induced collective motion of microtubules driven by kinesin. Nanoscale, 7(43):18054-18061, 2015.

[51] L. Ionov, A. Synytska, E. Kaul, and S. Diez. Protein-resistant polymer coatings based on surface-adsorbed poly (aminoethyl methacrylate)/poly (ethylene glycol) copolymers. Biomacromolecules, 11(1):233-237, 2009.

[52] R. E. Isele-Holder, J. Elgeti, and G. Gompper. Self-propelled worm-like filaments: spontaneous spiral formation, structure, and dynamics. Soft matter, 11(36):7181-7190, 2015.

[53] R. E. Isele-Holder, J. Jäger, G. Saggiorato, J. Elgeti, and G. Gompper. Dynamics of self-propelled filaments pushing a load. Soft Matter, 12(41):84958505, 2016.

[54] H. Ishikawa, J. Thompson, J. R. Yates Iii, and W. F. Marshall. Proteomic analysis of mammalian primary cilia. Current Biology, 22(5):414-419, 2012.

[55] K. Kaseda, H. Higuchi, and K. Hirose. Alternate fast and slow stepping of a heterodimeric kinesin molecule. Nature Cell Biology, 5(12):1079, 2003.

[56] F. C. Keber, E. Loiseau, T. Sanchez, S. J. DeCamp, L. Giomi, M. J. Bowick, M. C. Marchetti, Z. Dogic, and A. R. Bausch. Topology and dynamics of active nematic vesicles. Science, 345(6201):1135-1139, 2014.

[57] G. L. Kenausis, J. Vörös, D. L. Elbert, N. Huang, R. Hofer, L. Ruiz-Taylor, M. Textor, J. A. Hubbell, and N. D. Spencer. Poly (l-lysine)-g-poly (ethylene glycol) layers on metal oxide surfaces: attachment mechanism and effects of polymer architecture on resistance to protein adsorption. The Journal of Physical Chemistry B, 104(14):3298-3309, 2000. 
[58] D.-M. Kim and J. R. Swartz. Prolonging cell-free protein synthesis with a novel atp regeneration system. Biotechnology and bioengineering, 66(3):180$188,1999$.

[59] H. Kim, L. I. Binder, and J. L. Rosenbaum. The periodic association of map2 with brain microtubules in vitro. The Journal of cell biology, 80(2):266-276, 1979.

[60] S. Klumpp and R. Lipowsky. Cooperative cargo transport by several molecular motors. Proceedings of the National Academy of Sciences, 102(48):1728417289, 2005.

[61] S. Köhler and A. R. Bausch. Contraction mechanisms in composite active actin networks. PloS one, 7(7):e39869, 2012.

[62] O. Kratky and G. Porod. Röntgenuntersuchung gelöster fadenmoleküle. Recueil des Travaux Chimiques des Pays-Bas, 68(12):1106-1122, 1949.

[63] L. D. Landau, E. M. Lifshitz, et al. Theory of elasticity, volume 7. Pergamon Press, Oxford New York, 1986.

[64] C. B. Lindemann and K. A. Lesich. Flagellar and ciliary beating: the proven and the possible. J Cell Sci, 123(4):519-528, 2010.

[65] E. Mandelkow and E.-M. Mandelkow. Microtubules and microtubuleassociated proteins. Current opinion in cell biology, 7(1):72-81, 1995.

[66] T. Masuda, A. M. Akimoto, K. Nagase, T. Okano, and R. Yoshida. Artificial cilia as autonomous nanoactuators: Design of a gradient self-oscillating polymer brush with controlled unidirectional motion. Science advances, 2(8):e1600902, 2016.

[67] T. Mitchison and M. Kirschner. Dynamic instability of microtubule growth. nature, 312(5991):237, 1984.

[68] C. E. Monteith, M. E. Brunner, I. Djagaeva, A. M. Bielecki, J. M. Deutsch, and W. M. Saxton. A mechanism for cytoplasmic streaming: Kinesin-driven alignment of microtubules and fast fluid flows. Biophysical journal, 110(9):20532065, 2016.

[69] D. B. Murphy. Fundamentals of light microscopy and electronic imaging. John Wiley \& Sons, 2002. 
[70] D. B. Murphy, K. A. Johnson, and G. G. Borisy. Role of tubulin-associated proteins in microtubule nucleation and elongation. Journal of molecular biology, 117(1):33-52, 1977.

[71] F. Ndlec, T. Surrey, A. C. Maggs, and S. Leibler. Self-organization of microtubules and motors. Nature, 389(6648):305, 1997.

[72] F. Nédélec, T. Surrey, and A. Maggs. Dynamic concentration of motors in microtubule arrays. Physical review letters, 86(14):3192, 2001.

[73] D. Needleman and Z. Dogic. Active matter at the interface between materials science and cell biology. Nature Reviews Materials, 2(9):17048, 2017.

[74] D. J. Needleman, M. A. Ojeda-Lopez, U. Raviv, K. Ewert, J. B. Jones, H. P. Miller, L. Wilson, and C. R. Safinya. Synchrotron x-ray diffraction study of microtubules buckling and bundling under osmotic stress: a probe of interprotofilament interactions. Physical review letters, 93(19):198104, 2004.

[75] D. J. Needleman, M. A. Ojeda-Lopez, U. Raviv, K. Ewert, H. P. Miller, L. Wilson, and C. R. Safinya. Radial compression of microtubules and the mechanism of action of taxol and associated proteins. Biophysical journal, 89(5):3410-3423, 2005.

[76] N. T. Ouellette, H. Xu, and E. Bodenschatz. A quantitative study of threedimensional lagrangian particle tracking algorithms. Experiments in Fluids, 40(2):301-313, 2006.

[77] G. J. Pazour, N. Agrin, J. Leszyk, and G. B. Witman. Proteomic analysis of a eukaryotic cilium. J Cell Biol, 170(1):103-113, 2005.

[78] R. Phillips, J. Theriot, J. Kondev, and H. Garcia. Physical biology of the cell. Garland Science, 2012.

[79] D. Portran, J. Gaillard, M. Vantard, and M. Thery. Quantification of map and molecular motor activities on geometrically controlled microtubule networks. Cytoskeleton, 70(1):12-23, 2013.

[80] K. Prathyusha, S. Henkes, and R. Sknepnek. Dynamically generated patterns in dense suspensions of active filaments. Physical Review E, 97(2):022606, 2018.

[81] H. Qian, M. P. Sheetz, and E. L. Elson. Single particle tracking. analysis of diffusion and flow in two-dimensional systems. Biophysical journal, 60(4):910921, 1991. 
[82] R. Rezakhaniha, A. Agianniotis, J. T. C. Schrauwen, A. Griffa, D. Sage, C. v. Bouten, F. Van De Vosse, M. Unser, and N. Stergiopulos. Experimental investigation of collagen waviness and orientation in the arterial adventitia using confocal laser scanning microscopy. Biomechanics and modeling in mechanobiology, 11(3-4):461-473, 2012.

[83] I. H. Riedel-Kruse, A. Hilfinger, J. Howard, and F. Jülicher. How molecular motors shape the flagellar beat. HFSP journal, 1(3):192-208, 2007.

[84] W. H. Roos, O. Campas, F. Montel, G. Woehlke, J. P. Spatz, P. Bassereau, and G. Cappello. Dynamic kinesin-1 clustering on microtubules due to mutually attractive interactions. Physical biology, 5(4):046004, 2008.

[85] L. Ruiz-Taylor, T. Martin, F. Zaugg, K. Witte, P. Indermuhle, S. Nock, and P. Wagner. Monolayers of derivatized poly (l-lysine)-grafted poly (ethylene glycol) on metal oxides as a class of biomolecular interfaces. Proceedings of the National Academy of Sciences, 98(3):852-857, 2001.

[86] N. Saitô, K. Takahashi, and Y. Yunoki. The statistical mechanical theory of stiff chains. Journal of the Physical Society of Japan, 22(1):219-226, 1967.

[87] G. Salbreux, G. Charras, and E. Paluch. Actin cortex mechanics and cellular morphogenesis. Trends in cell biology, 22(10):536-545, 2012.

[88] T. Sanchez, D. T. Chen, S. J. DeCamp, M. Heymann, and Z. Dogic. Spontaneous motion in hierarchically assembled active matter. Nature, 491(7424):431, 2012.

[89] T. Sanchez, D. Welch, D. Nicastro, and Z. Dogic. Cilia-like beating of active microtubule bundles. Science, 333(6041):456-459, 2011.

[90] A. Seitz and T. Surrey. Processive movement of single kinesins on crowded microtubules visualized using quantum dots. The EMBO journal, 25(2):267$277,2006$.

[91] K. Sekimoto, N. Mori, K. Tawada, and Y. Y. Toyoshima. Symmetry breaking instabilities of an in vitro biological system. Physical review letters, 75(1):172, 1995.

[92] M. P. Sheetz, R. Chasan, and J. A. Spudich. Atp-dependent movement of myosin in vitro: characterization of a quantitative assay. The Journal of cell biology, 99(5):1867-1871, 1984. 
[93] M. P. Sheetz and J. A. Spudich. Movement of myosin-coated fluorescent beads on actin cables in vitro. Nature, 303(5912):31, 1983.

[94] M. B. Smith, H. Li, T. Shen, X. Huang, E. Yusuf, and D. Vavylonis. Segmentation and tracking of cytoskeletal filaments using open active contours. Cytoskeleton, 67(11):693-705, 2010.

[95] E. D. Spoerke, A. K. Boal, G. D. Bachand, and B. C. Bunker. Templated nanocrystal assembly on biodynamic artificial microtubule asters. ACS nano, 7(3):2012-2019, 2013.

[96] R. Subramanian and J. Gelles. Two distinct modes of processive kinesin movement in mixtures of atp and amp-pnp. The Journal of general physiology, 130(5):445-455, 2007.

[97] T. Surrey, F. Nédélec, S. Leibler, and E. Karsenti. Physical properties determining self-organization of motors and microtubules. Science, 292(5519):11671171, 2001.

[98] K. Suzuki, M. Miyazaki, J. Takagi, T. Itabashi, and S. Ishiwata. Spatial confinement of active microtubule networks induces large-scale rotational cytoplasmic flow. Proceedings of the National Academy of Sciences, 114(11):29222927, 2017.

[99] I. A. Telley, P. Bieling, and T. Surrey. Obstacles on the microtubule reduce the processivity of kinesin-1 in a minimal in vitro system and in cell extract. Biophysical journal, 96(8):3341-3353, 2009.

[100] W. Thielicke and E. Stamhuis. Pivlab-towards user-friendly, affordable and accurate digital particle image velocimetry in matlab. Journal of Open Research Software, 2(1), 2014.

[101] P. K. Trong, J. Guck, and R. E. Goldstein. Coupling of active motion and advection shapes intracellular cargo transport. Physical review letters, 109(2):028104, 2012.

[102] R. D. Vale, T. Funatsu, D. W. Pierce, L. Romberg, Y. Harada, and T. Yanagida. Direct observation of single kinesin molecules moving along microtubules. Nature, 380(6573):451, 1996.

[103] A. Vilfan, E. Frey, F. Schwabl, M. Thormählen, Y.-H. Song, and E. Mandelkow. Dynamics and cooperativity of microtubule decoration by the motor protein kinesin. Journal of molecular biology, 312(5):1011-1026, 2001. 
[104] A. Vilfan, S. Subramani, E. Bodenschatz, R. Golestanian, and I. Guido. Flagella-like beating of a single microtubule. Nano Letters, 19(5):3359-3363, 2019. PMID: 30998020.

[105] M. Vilfan, A. Potočnik, B. Kavčič, N. Osterman, I. Poberaj, A. Vilfan, and D. Babič. Self-assembled artificial cilia. Proceedings of the National Academy of Sciences, 107(5):1844-1847, 2010.

[106] H. H. Wensink, J. Dunkel, S. Heidenreich, K. Drescher, R. E. Goldstein, H. Löwen, and J. M. Yeomans. Meso-scale turbulence in living fluids. Proceedings of the National Academy of Sciences, 109(36):14308-14313, 2012.

[107] E. C. Young, E. Berliner, H. K. Mahtani, B. Perez-Ramirez, and J. Gelles. Subunit interactions in dimeric kinesin heavy chain derivatives that lack the kinesin rod. Journal of Biological Chemistry, 270(8):3926-3931, 1995. 


\section{Acknowledgements}

I would like to thank my PhD advisor Dr. Isabella Guido for her valuable guidance and constant support through the years in moulding me towards embarking on scientific career. I am very grateful for the hours that were spent discussing my experimental observations and correcting this thesis. I thank Prof. Dr. Eberhard Bodenschatz for providing me the opportunity to explore an inter-disciplinary field in my $\mathrm{PhD}$ project and attend mind-stimulating conferences. I thank the members of my thesis committee, Prof. Dr. Joerg Enderlein and Prof. Dr. Stefan Klumpp for juggling their hectic schedules to make time for the TAC meetings and offering useful advice towards the completion of this work. I sincerely thank the technical staff in the lab, especially Sandra Klama and Sarah Romanowski for fulfilling my never-ending demands of chemical aliquots and passivated surfaces.

I am indebted to the GGNB team of Frauke Bergmann and Antje Erdmann for answering and helping me endlessly, right from my GGNB admission to the submission of this thesis. I acknowledge financial support from the IMPRS Excellence stipend and the MaxSynBio Network. I also thank Angela Meister for easing the administrative burden and selflessly helping me when I first arrived in Göttingen.

I also thank Dr. Michael Wilczek, Dr. Narain Karedla, Dr. Azam Gholami, Dr. Albert Bae, Dr. K.R. Prathyusha and Dr. Andrej Vilfan for their technical guidance and providing me with sources for various concepts that were unclear to me.

Special thanks goes to Francesca Cavallini, Dr. Hyejeong Kim, Dr. Kaumudi Prabhakara and Dr. Laura Turco for being like sisters : All the fun memories, their concern and constant words of encouragement meant a lot to me. I'd also like to thank Dr. Christian Westendorf for the elaborate microscopy training and being the co-founder of the 'quirky dance moves' club that I'm a proud member of. I thank all my colleagues and office-mates for their encouragement and the entertaining coffee conversations that I managed to join once a month. I also thank my family, parents and Raison Dsouza for inspiring me to be passionate and level-headed in this journey. 


\section{Curriculum Vitae}

\section{Smrithika Subramani}

Date of Birth : 05.03.1993

\section{Academical Background :}

$09.2015-10.2019$

\section{Doctoral Dissertation}

Self - organization of microtubules for biomimetic structures

Max Planck Institute for Dynamics and Self-organization, Göttingen, Germany

Dr. Isabella Guido

$07.2013-05.2015$

Master of Science (Physics)

National Institute of Technology Karnataka, India

$08.2014-05.2015$

\section{MSc Thesis}

Synthesis, characterization and electrochemical studies of graphene- $\mathrm{ZnO}$ nanocomposites

National Institute of Technology Karnataka, India

Prof. Dr. H.S. Nagaraja

$05.2014-07.2014$

\section{Research Internship}

Transport studies of reduced Graphene oxide using LabVIEW

Indian Institute of Technology Madras, Chennai, India

\section{Talks and Posters :}

EMBO Symposia on Microtubules : From Atoms to Complex Systems (2016 and 2018)

Heidelberg, Germany

Poster : "Synthetic Reconstitution of beating cilia"

DPG Spring meeting 2018

Berlin, Germany

Talk: "Dynamics of synthetically assembled microtubules and motors" 
Third Infinity $2017: 3^{\text {rd }}$ conference on Physics of Biological and Complex Systems

Göttingen, Germany

Talk : "Bio-inspired beating structures"

\section{Teaching experience :}

Undergraduate thesis supervision on characterization of active microtubule networks

Teaching Assistant (Physics Department, University of Göttingen)

- Quantum Mechanics

- Solid State Physics

\section{Awards :}

- International Max Planck Research School (IMPRS) fellow of the Physics of Biological and Complex Systems (PBCS) school of Max Planck Society (2015-2018)

- Poster prize winner at Symposium Engineering Life 2017: Form and Function organized at Center for Molecular Bioengineering, TU Dresden

\section{Publications :}

" Flagella-like Beating of a Single Microtubule" , Andrej Vilfan* Smrithika Subramani*, Eberhard Bodenschatz, Ramin Golestanian and Isabella Guido, Nano Letters 2019 19(5), 3359-3363 (* equal contribution) 
